\title{
Complexation of $\boldsymbol{C}$-Functionalized Cyclams with Copper(II) and Zinc(II): Similarities and Changes When Compared to Parent Cyclam Analogues
}

Evan Lelong, ${ }^{\dagger}$ Jong-Min Suh, ${ }^{ \pm}$Gunhee Kim, ${ }^{ \pm}$David Esteban Gòmez, ${ }^{\perp}$ Marie Cordier, ${ }^{\approx}$ Mi Hee Lim $_{,}^{ \pm}$Rita Delgado, ${ }^{\square}$ Guy Royal, ${ }^{\ddagger}$ Carlos Platas-Iglesias, ${ }^{\perp, *}$ Hélène Bernard ${ }^{\dagger}$ and Raphaël Tripier ${ }^{\dagger, *}$

UUniv Brest, UMR CNRS 6521 CEMCA, 6 avenue Victor le Gorgeu, 29238 Brest, France. Email: raphael.tripier@univ-brest.fr

${ }^{\perp}$ Departamento de Química, Facultade de Ciencias \& Centro de Investigaciones Científicas Avanzadas (CICA), Universidade da Coruña, 15071 A Coruña, Spain. Email: carlos.platas.iglesias@udc.es

$\square$ Instituto de Tecnologia Química e Biológica António Xavier, Universidade Nova de Lisboa, Av. da República, 2780-157 Oeiras, Portugal. Email: delgado@itqb.unl.pt

\#niversité Grenoble Alpes, CNRS, Département de Chimie Moléculaire (UMR5250), F38400

Grenoble, France. Email: guy.royal@univ-grenoble-alpes.fr

${ }^{ \pm}$Department of Chemistry, Korea Advanced Institute of Science and Technology (KAIST),

Daejeon 34141, Republic of Korea. Email: miheelim@kaist.ac.kr

Univ Rennes, CNRS, ISCR (Institut des Sciences Chimiques de Rennes) UMR $6226 \backslash$ F-35000

Rennes, France. Email: marie.dallon@univ-rennes1.fr 


\section{Table of Contents}

Glossary of chart $1 \quad$ S6

$\begin{array}{ll}\text { Synthesis and characterization of the metal complexes } & \text { S7 }\end{array}$

Figure S1. $\quad$ NMR ${ }^{13} \mathrm{C}$ Jmod spectra of anti and syn isomers of CB-DMC-E. $\quad$ S13

Figure S2. $\quad$ NMR ${ }^{1} \mathrm{H}$ Jmod spectra of anti and syn isomers of CB-DMC-E. $\quad$ S13

Condition used to obtained crystals discussed in the main manuscript $\quad$ S14

Figure S3. View of the crystal structure of CB-DMC-E diastereoisomers. $\quad$ S14

Table S1. $\quad$ Crystallographic data of CB-DMC-E. S15

Figure S4. $\quad$ ESI-MS spectrum of $\left[\mathrm{Cu}(\right.$ cyclam $\left.)(\mathrm{Cl})_{2}\right] . \quad \mathrm{S} 16$

$\begin{array}{lll}\text { Figure S5. } & \text { ESI-MS spectrum of }\left[\mathrm{Zn}(\text { cyclam })(\mathrm{Cl})_{2}\right] . & \mathrm{S} 16\end{array}$

$\begin{array}{llr}\text { Figure S6. } & \text { ESI-HRMS spectrum of } \mathrm{Cu}(\mathbf{D M C})^{2+} & \mathrm{S} 17\end{array}$

$\begin{array}{llr}\text { Figure S7. } & \text { ESI-HRMS spectrum of } \mathrm{Zn}(\mathbf{D M C})^{2+} & \mathrm{S} 17\end{array}$

$\begin{array}{llr}\text { Figure S8. } & \text { ESI-MS spectrum of }\left[\mathrm{Cu}(\mathbf{T M C})(\mathrm{Cl})_{2}\right] . & \mathrm{S} 18\end{array}$

$\begin{array}{llr}\text { Figure S9. } & \text { ESI-MS spectrum of }\left[\mathrm{Zn}(\mathbf{T M C})(\mathrm{Cl})_{2}\right] . & \mathrm{S} 18\end{array}$

$\begin{array}{llr}\text { Figure S10. } & \text { ESI-HRMS spectrum of } \mathrm{Cu}(\mathbf{C B}-\text { cyclam })^{2+} & \mathrm{S} 19\end{array}$

Figure S11. ESI-HRMS spectrum of Zn(CB-cyclam $)^{2+}$ S19

$\begin{array}{lll}\text { Figure S12. } & \text { ESI-MS spectrum of } \mathrm{Cu}(\mathbf{C B}-\mathrm{DMC})^{2+} & \text { S20 }\end{array}$

Figure S13. $\quad$ ESI-HRMS spectrum of Zn(CB-DMC $)^{2+} . \quad$ S20

Figure S14. $\quad$ ESI-HRMS spectrum of $\mathrm{Cu}(\text { cyclam-E })^{+} . \quad$ S21

Figure S15. $\quad$ ESI-HRMS spectrum of Zn(cyclam-E) ${ }^{2+} . \quad$ S21

Figure S16. $\quad$ ESI-HRMS spectrum of $\mathrm{Cu}(\mathbf{D M C}-\mathbf{E})^{2+}{ }^{2+} \quad$ S22

Figure S17. ESI-HRMS spectrum of compound Zn(DMC-E) $)^{2+}$ S22

Figure S18. $\quad$ ESI-HRMS spectrum of $\mathrm{Cu}(\mathbf{T M C}-\mathbf{E})^{2+}$ S23

Figure S19. $\quad$ ESI-HRMS spectrum of Zn(TMC-E) $)^{2+} . \quad$ S23

Figure S20. $\quad$ ESI-HRMS spectrum of compound $\mathrm{Cu}(\mathbf{C B}-\mathbf{c y c l a m}-\mathbf{E})^{2+} . \quad \mathrm{S} 24$ 
Figure S21. ESI-HRMS spectrum of compound $\mathrm{Zn}(\mathbf{C B}-\mathbf{c y c l a m}-\mathbf{E})^{2+}$.

Figure S22. $\quad$ ESI-HRMS spectrum of compound $\mathrm{Cu}(\mathbf{C B}-\mathbf{D M C}-\mathbf{E})^{2+} . \quad$ S25

Figure S23. $\quad$ ESI-HRMS spectrum of compound Zn(CB-DMC-E $)^{2+} . \quad$ S25

Figure S24. Crystal structures of $\left[\mathrm{Cu}(\right.$ cyclam-E $\left.)\left(\mathrm{ClO}_{4}\right)_{2}\right]$ and $\left[\mathrm{Cu}(\mathbf{c y c l a m})\left(\mathrm{ClO}_{4}\right)_{2}\right] . \quad \mathrm{S} 26$

Figure S25. Crystal structures of $\left.\left[\mathrm{Cu}(\mathbf{D M C})(\mathrm{Cl})_{2}\right)\right]$ and $\left.\left[\mathrm{Zn}(\mathbf{D M C})(\mathrm{Cl})_{2}\right)\right] . \quad$ S26

Table S2. $\quad$ Crystallographic data of $\left[(\mathrm{CuDMC}-\mathrm{E})_{2}(\mathrm{Cl})\left(\mathrm{NO}_{3}\right)\right]\left(\mathrm{NO}_{3}\right)_{2} . \quad \mathrm{S} 27$

Table S3. $\quad$ Crystallographic data of $[\mathrm{Cu}(\mathbf{T M C}-\mathbf{E}) \mathrm{Cl}] \mathrm{NO}_{3} . \quad$ S28

Table S4. $\quad$ Crystallographic data of $[\mathrm{Zn}(\mathbf{T M C}-\mathbf{E}) \mathrm{Cl}] \mathrm{NO}_{3} . \quad$ S29

Figure S26. Superposition of the crystal structures of [Cu(TMC)(NCS)] / $[\mathrm{Cu}(\mathbf{T M C}-\mathbf{E})(\mathrm{Cl})]^{+}$and $[\mathrm{Zn}(\mathbf{T M C}) \mathrm{Cl}]^{+} /[\mathrm{Zn}(\mathbf{T M C}-\mathbf{E})(\mathrm{Cl})] . \quad \mathrm{S} 30$

Table S5. $\quad$ Crystallographic data of $\left[(\mathrm{ZnCB}-\mathbf{c y c l a m}-\mathrm{E})_{2}(\mathrm{Cl})_{2}\right] \mathrm{Cl}_{2} . \quad \mathrm{S} 31$

Figure 27. Superposition of the structures of $[\mathrm{Zn}(\mathbf{C B}-\mathbf{c y c l a m})(\mathrm{Cl})]^{+}$with cis- $[\mathrm{Zn}(\mathbf{C B}-\text { cyclam-E })(\mathrm{Cl})]^{+}$and anti-[Zn(CB-cyclam-E $\left.)(\mathrm{Cl})\right]^{+} . \quad \mathrm{S} 32$

Figure S28. $\quad \mathrm{NMR}{ }^{13} \mathrm{C}$ Jmod and ${ }^{1} \mathrm{H}$ spectra of $\left[\mathrm{Zn}(\right.$ cyclam $\left.) \mathrm{Cl}_{2}\right] . \quad \mathrm{S} 33$

Figure S29. $\quad \mathrm{NMR}{ }^{13} \mathrm{C}$ Jmod and ${ }^{1} \mathrm{H}$ spectra of [Zn(cyclam-E) $\left.\mathrm{Cl}_{2}\right] . \quad \mathrm{S} 34$

Figure S30. $\quad \mathrm{NMR}{ }^{13} \mathrm{C}$ Jmod and ${ }^{1} \mathrm{H}$ spectra of $\left[\mathrm{Zn}(\mathbf{D M C}) \mathrm{Cl}_{2}\right] . \quad \mathrm{S} 35$

Figure S31. $\quad \mathrm{NMR}{ }^{13} \mathrm{C}$ Jmod and ${ }^{1} \mathrm{H}$ spectra of $\left[\mathrm{Zn}(\mathbf{D M C}-\mathbf{E}) \mathrm{Cl}_{2}\right] . \quad \mathrm{S} 36$

Figure S32. $\quad \mathrm{NMR}{ }^{13} \mathrm{C}$ Jmod and ${ }^{1} \mathrm{H}$ spectra of $\left[\mathrm{Zn}(\mathbf{T M C}) \mathrm{Cl}_{2}\right] . \quad \mathrm{S} 37$

Figure S33. $\quad \mathrm{NMR}{ }^{13} \mathrm{C}$ Jmod and ${ }^{1} \mathrm{H}$ spectra of [Zn(TMC-E) $\left.\mathrm{Cl}_{2}\right] . \quad \mathrm{S} 37$

Figure S34. 2D COSY ${ }^{1} \mathrm{H}_{-}{ }^{1} \mathrm{H}$ NMR spectrum of $\left[\mathrm{Zn}(\mathbf{T M C}-\mathbf{E}) \mathrm{Cl}_{2}\right] . \quad \mathrm{S} 38$

Figure S35. 2D HMQC ${ }^{1} \mathrm{H}_{-}{ }^{13} \mathrm{C}$ NMR spectrum of $\left[\mathrm{Zn}(\mathbf{T M C}-\mathbf{E}) \mathrm{Cl}_{2}\right] . \quad \mathrm{S} 38$

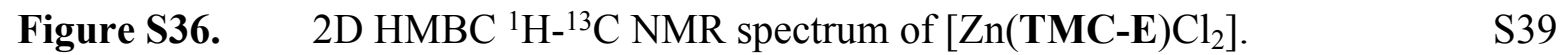

Table S6. $\quad{ }^{13} \mathrm{C}$ and ${ }^{1} \mathrm{H}$ NMR shifts of $\left[\mathrm{Zn}(\mathbf{T M C}-\mathbf{E}) \mathrm{Cl}_{2}\right] . \quad \mathrm{S} 39$

Figure S37. $\quad \mathrm{NMR}{ }^{13} \mathrm{C}$ Jmod and ${ }^{1} \mathrm{H}$ spectra of $\left[\mathrm{Zn}(\mathbf{C B}\right.$-cyclam $\left.) \mathrm{Cl}_{2}\right] . \quad \mathrm{S} 40$

Figure S38. $\quad \mathrm{NMR}{ }^{13} \mathrm{C}$ Jmod and ${ }^{1} \mathrm{H}$ spectra of $\left[\mathrm{Zn}(\mathbf{C B}-\mathbf{c y c l a m}-\mathbf{E}) \mathrm{Cl}_{2}\right] . \quad \mathrm{S} 40$ 
Figure S39. $\quad \mathrm{NMR}{ }^{13} \mathrm{C}$ Jmod and ${ }^{1} \mathrm{H}$ spectra of $\left[\mathrm{Zn}(\mathbf{C B}-\mathbf{D M C}) \mathrm{Cl}_{2}\right]$.

Figure S40. NMR ${ }^{13} \mathrm{C}$ Jmod and ${ }^{1} \mathrm{H}$ spectra of anti-[Zn(CB-DMC-E) $\left.\mathrm{Cl}_{2}\right]$.

Figure S41. $\quad \mathrm{NMR}{ }^{13} \mathrm{C}$ Jmod and ${ }^{1} \mathrm{H}$ spectra of $s y n-\left[\mathrm{Zn}(\mathbf{C B}-\mathbf{D M C}-\mathbf{E}) \mathrm{Cl}_{2}\right]$.

Figure S42. Visible spectra of $\left[\mathrm{Cu}(\right.$ cyclam $\left.) \mathrm{Cl}_{2}\right],\left[\mathrm{Cu}(\right.$ cyclam-E) $\left.) \mathrm{Cl}_{2}\right]$ $\left[\mathrm{Cu}(\mathbf{D M C}) \mathrm{Cl}_{2}\right],\left[\mathrm{Cu}(\mathbf{D M C}-\mathbf{E}) \mathrm{Cl}_{2}\right],\left[\mathrm{Cu}(\mathbf{T M C}) \mathrm{Cl}_{2}\right]$ and $\left[\mathrm{Cu}(\mathbf{T M C}-\mathbf{E}) \mathrm{Cl}_{2}\right]$.

Figure S43. Visible spectra of $\left[\mathrm{Cu}(\mathbf{C B}-\right.$ cyclam $\left.) \mathrm{Cl}_{2}\right],[\mathrm{Cu}(\mathbf{C B}-\mathbf{c y c l a m}-$

E) $\left.\mathrm{Cl}_{2}\right],\left[\mathrm{Cu}(\mathbf{C B}-\mathbf{D M C}) \mathrm{Cl}_{2}\right]$ and $\left[\mathrm{Cu}(\mathbf{C B}-\mathbf{D M C}-\mathbf{E}) \mathrm{Cl}_{2}\right]$.

Figure S44. Visible spectra of acid-mediated dissociation of

$\mathrm{Cu}(\text { cyclam })^{2+}, \mathrm{Cu}(\text { cyclam-E })^{2+}, \mathrm{Cu}(\mathbf{D M C})^{2+}, \mathrm{Cu}(\mathbf{D M C}-$ $\mathbf{E})^{2+}$, trans-III-Cu(TMC) ${ }^{2+}$ and trans-III-Cu(TMC-E) $)^{2+}$.

Figure S45. UV-Visible spectra of acid-mediated dissociation of $\mathrm{Cu}(\mathbf{C B}-\text { cyclam })^{2+}, \mathrm{Cu}(\mathbf{C B}-\text { cyclam-E })^{2+}, \mathrm{Cu}(\mathbf{C B}-\mathbf{D M C})^{2+}$ and $\mathrm{Cu}(\mathbf{C B}-\mathbf{D M C}-\mathbf{E})^{2+}$.

Figure S46. Cyclic voltammograms of $\mathrm{Cu}(\mathbf{D M C})^{2+}$ and $\mathrm{Cu}(\mathbf{D M C}-\mathbf{E})^{2+}$.

Figure S47. Cyclic voltammograms of $\mathrm{Cu}(\text { cyclam-E })^{2+}$ and $\mathrm{Cu}(\text { cyclam })^{2+}$.

Table S7. Cartesian coordinates of $[\mathrm{Cu}(\mathbf{c y c l a m}) \mathrm{Cl}]^{+}($trans-III). S47

Table S8. Cartesian coordinates of $\left[\mathrm{Cu}(\text { cyclam })\left(\mathrm{H}_{2} \mathrm{O}\right)\right]^{2+}($ trans-III). S48

Table S9. Cartesian coordinates of $\left[\mathrm{Cu}(\text { cyclam-E })\left(\mathrm{H}_{2} \mathrm{O}\right)\right]^{2+}($ trans-III).

Table S10. Cartesian coordinates of $\left[\mathrm{Cu}(\mathbf{D M C})\left(\mathrm{H}_{2} \mathrm{O}\right)\right]^{2+}($ trans-III).

Table S11. Cartesian coordinates of $\left[\mathrm{Cu}(\mathbf{D M C}-\mathbf{E})\left(\mathrm{H}_{2} \mathrm{O}\right)\right]^{2+}$ (trans-III).

Table S12. Cartesian coordinates of $\left[\mathrm{Cu}(\mathbf{T M C})\left(\mathrm{H}_{2} \mathrm{O}\right)\right]^{2+}$ (trans-I). S52

Table S13. Cartesian coordinates of $\left[\mathrm{Cu}(\mathbf{C B}-\mathbf{c y c l a m})\left(\mathrm{H}_{2} \mathrm{O}\right)\right]^{2+}$.

Table S14. Cartesian coordinates of $[\mathrm{Cu}(\mathbf{C B}-\mathbf{c y c l a m})(\mathrm{Cl})]^{+}$.

Table S15. Cartesian coordinates of $[\mathrm{Cu}(\mathbf{C B}-\mathbf{c y c l a m}-\mathbf{E})(\mathrm{Cl})]^{+}$.

Table S16. Cartesian coordinates of $\left[\mathrm{Cu}(\mathbf{C B}-\mathbf{c y c l a m}-\mathbf{E})\left(\mathrm{H}_{2} \mathrm{O}\right)\right]^{2+}$.

Table S17. Cartesian coordinates of $[\mathrm{Cu}(\mathrm{CB}-\mathrm{DMC})(\mathrm{Cl})]^{+}$.

Table S18. Cartesian coordinates of $\left[\mathrm{Cu}(\mathbf{C B}-\mathrm{DMC})\left(\mathrm{H}_{2} \mathrm{O}\right)\right]^{2+}$.

Table S19. Cartesian coordinates of $[\mathrm{Cu}(\mathbf{C B}-\mathrm{DMC}-\mathbf{E})(\mathrm{Cl})]^{+}$. 
Table S20. $\quad$ Cartesian coordinates of $\left[\mathrm{Cu}(\mathbf{C B}-\mathbf{D M C}-\mathbf{E})\left(\mathrm{H}_{2} \mathrm{O}\right)\right]^{2+} . \quad \mathrm{S} 61$

Table S21. Cartesian coordinates of $[\mathrm{Zn}(\mathbf{D M C}) \mathrm{Cl}]^{+}$(trans-I). S62

Table S22. $\quad$ Cartesian coordinates of $[\mathrm{Zn}(\mathbf{D M C}) \mathrm{Cl}]^{+}($trans-III). $\quad$ S64

Table S23. Cartesian coordinates of $[\mathrm{Zn}(\mathbf{D M C}) \mathrm{Cl}]^{+}($cis- $) . \quad$ S65

Table S24. Cartesian coordinates of $[\mathrm{Zn}(\mathbf{c y c l a m}) \mathrm{Cl}]^{+}($trans $-\mathrm{I}) . \quad$ S66

Table S25. Cartesian coordinates of $[\mathrm{Zn}(\mathbf{c y c l a m}) \mathrm{Cl}]^{+}($trans-III $) . \quad$ S67

Table S26. $\quad$ Cartesian coordinates of $[\mathrm{Zn}(\text { cyclam }) \mathrm{Cl}]^{+}($cis- $-\mathrm{V}) . \quad$ S68

Table S27. Cartesian coordinates of $[\mathrm{Zn}(\mathbf{T M C}) \mathrm{Cl}]^{+}($trans-I). $\quad$ S69

Table S28. $\quad$ Cartesian coordinates of $[\mathrm{Zn}(\mathbf{T M C}) \mathrm{Cl}]^{+}($trans-III). $\quad$ S70

Table S29. $\quad$ Cartesian coordinates of $[\mathrm{Zn}(\mathbf{T M C}) \mathrm{Cl}]^{+}($cis- $-\mathrm{V}) . \quad$ S71

Table S30. $\quad$ Cartesian coordinates of $[\mathrm{Zn}(\mathbf{C B}-\mathbf{c y c l a m}-\mathbf{E}) \mathrm{Cl}]^{+}($anti $) . \quad \mathrm{S} 72$

Table S31. Cartesian coordinates of $[\mathrm{Zn}(\mathbf{C B}-\mathbf{c y c l a m}-\mathbf{E}) \mathrm{Cl}]^{+}($syn $) . \quad \mathrm{S} 73$

Table S32. $\quad$ Cartesian coordinates of $[\mathrm{Zn}(\mathbf{C B}-\mathrm{DMC}-\mathbf{E}) \mathrm{Cl}]^{+}($anti $) . \quad$ S74

Table S33. Cartesian coordinates of $[\mathrm{Zn}(\mathbf{C B}-\mathrm{DMC}-\mathbf{E}) \mathrm{Cl}]^{+}($syn $) . \quad$ S76

$\begin{array}{ll}\text { References } & \text { S77 }\end{array}$ 


\section{Glossary of chart 1}<smiles>C1CNCCNCCNCCNC1</smiles>

cyclam<smiles>CN1CCCNCCN(C)CCCNCC1</smiles>

DMC<smiles>CN1CCCN(C)CCCN(C)CCC1</smiles>

TMC<smiles>NCCCNCCCN1CCCCNCC1</smiles>

CB-cyclam<smiles>CN1CCCN(CCCN2CCCN(C)CC2)CC1</smiles>

CB-DMC<smiles>OCCC1CNCCNCCCNCCNC1</smiles>

cyclam-E<smiles>CN1CCNCCCN(C)CC(CCO)CNCC1</smiles>

DMC-E<smiles>CN1CCCN(C)CCN(C)CC(CCO)CN(C)CC1</smiles>

TMC-E<smiles>OCCC1CCN2CCNCCCN(CCNC1)C2</smiles><smiles>CN1CCCN(CCN2CCN(C)CCCN(C)CC(CCO)C2)CC1</smiles>

CB-cyclam-E CB-DMC-E

Cyclam: 1,4,8,11-tetraazacyclotetradecane

DMC: 1,8-dimethyl-1,4,8,11-tetraazacyclotetradecane

TMC: 1,4,8,11-tetramethyl-1,4,8,11-tetraazacyclotetradecane

CB-cyclam: 1,4,8,11-tetraazabicyclo[6.6.2]hexadecane

CB-DMC: 4,11-dimethyl-1,4,8,11-tetraazabicyclo[6.6.2]hexadecane cyclam-E: 2-(1,4,8,11-tetraazacyclotetradec-6-yl)ethanol DMC-E: 2-(1,8-dimethyl-1,4,8,11-tetraazacyclotetradec-6-yl)ethanol TMC-E: 2-(1,4,8,11-tetramethyl-1,4,8,11-tetraazacyclotetradec-6-yl)ethanol CB-cyclam-E: 2-(1,4,8,11-tetraazabicyclo[6.6.2]hexadec-6-yl)ethanol CB-DMC-E: 2-(4,11-dimethyl-1,4,8,11-tetraazabicyclo[6.6.2]hexadec-6-yl)ethanol. 


\section{Synthesis and characterization of the metal complexes}

The general procedure of synthesis is reported in the experimental section of the main manuscript.

\section{Copper(II) 1,4,8,11-tetrazacyclotetradecane dichloride}

Cyclam (39.5 mg, 0.198mmol), $\mathrm{CuCl}_{2}(29.2 \mathrm{mg}, 0.217 \mathrm{mmol})$.

Complex was obtained as pink powder (49 mg, $74 \%)$.

ESI-MS (positive, $\mathrm{H}_{2} \mathrm{O} / \mathrm{CH}_{3} \mathrm{CN} 50 / 50$ ): $\mathrm{m} / z$ found $131.552\left[\mathrm{Cu}\right.$ (cyclam) ${ }^{2+}$, found 262.098 $[\mathrm{Cu}(\text { cyclam })-\mathrm{H}]^{+}$, found $298.074[\mathrm{Cu}(\text { cyclam })+\mathrm{Cl}]^{+}$.

\section{Zinc(II) 1,4,8,11-tetrazacyclotetradecane dichloride}

Cyclam (39.5 mg, $0.198 \mathrm{mmol}), \mathrm{ZnCl}_{2}(29.8 \mathrm{mg}, 0.217 \mathrm{mmol})$.

Complex was obtained as a white powder (45 mg, $67 \%)$.

ESI-MS (positive, $\mathrm{H}_{2} \mathrm{O} / \mathrm{CH}_{3} \mathrm{CN} 50 / 50$ ): $\mathrm{m} / z$ found 132.0530 [Zn(cyclam)] ${ }^{2+}$, found 299.0708 $[\mathrm{Zn}(\text { cyclam })+\mathrm{Cl}]^{+}$, found $309.1017[\mathrm{Zn}(\text { cyclam })+\mathrm{HCOO}]^{+} .{ }^{1} \mathbf{H} \mathbf{~ N M R}\left(300 \mathrm{MHz}, \mathrm{D}_{2} \mathrm{O}, 298 \mathrm{~K}\right)$ : trans-III: $\delta=3.11-3.07(\mathrm{~m}, 4 \mathrm{H}), 2.94-2.91(\mathrm{~m}, 4 \mathrm{H}), 2.67-2.59(\mathrm{~m}, 4 \mathrm{H}), 2.41-2.39(\mathrm{~m}, 4 \mathrm{H}), 1.83-$ $1.78(\mathrm{~m}, 2 \mathrm{H}), 1.62-1.53 \mathrm{ppm}(\mathrm{m}, 2 \mathrm{H}) . \underline{\text { trans }-\mathrm{I}}$ and $\underline{\mathrm{cis}-\mathrm{V}}$ are unidentifiable (See below). ${ }^{13} \mathrm{C}$ NMR $\left(75 \mathrm{MHz}, \mathrm{D}_{2} \mathrm{O}, 298 \mathrm{~K}\right)$ : trans-III: $\delta=52.7\left(4 \mathrm{C}, \mathrm{N}_{-} \underline{\mathrm{CH}}_{2} \mathrm{CH}_{2} \underline{\mathrm{CH}_{2}}-\mathrm{N}\right), 50.4(4 \mathrm{C}, \mathrm{N}-$ $\left.\underline{\mathrm{CH}}_{2} \mathrm{CH}_{2}-\mathrm{N}\right), 30.7$ ppm $\left(2 \mathrm{C}, \mathrm{CH}_{2}-\beta-\mathrm{N}\right)$. trans-I: $\delta=53.3\left(4 \mathrm{C}, \mathrm{N}-\underline{\mathrm{CH}}_{2} \mathrm{CH}_{2} \underline{\mathrm{CH}}_{2}-\mathrm{N}\right), 49.1(4 \mathrm{C}, \mathrm{N}-$ $\left.\underline{\mathrm{CH}}_{2} \underline{\mathrm{CH}}_{2}-\mathrm{N}\right), 30.2 \mathrm{ppm}\left(2 \mathrm{C}, \mathrm{CH}_{2}-\beta-\mathrm{N}\right) . \underline{c i s}-\mathrm{V}: \delta=51.8\left(4 \mathrm{C}, \mathrm{N}-\underline{\mathrm{CH}}_{2} \mathrm{CH}_{2} \underline{\mathrm{CH}}_{2}-\mathrm{N}\right), 48.8(4 \mathrm{C}, \mathrm{N}-$ $\left.\underline{\mathrm{CH}}_{2} \underline{\mathrm{CH}}_{2}-\mathrm{N}\right), 26.5 \mathrm{ppm}\left(2 \mathrm{C}, \mathrm{CH}_{2}-\beta-\mathrm{N}\right)$.

\section{Copper(II) 1,8-dimethyl-1,4,8,11-tetrazacyclotetradecane dichloride}

DMC (41.1 mg, $0.180 \mathrm{mmol}), \mathrm{CuCl}_{2}(26.6 \mathrm{mg}, 0.198 \mathrm{mmol})$.

Complex was obtained as a purple powder $(51.0 \mathrm{mg}, 78 \%)$.

ESI-HR-MS (positive, $\mathrm{H}_{2} \mathrm{O}$ ): $\mathrm{m} / z$ calcd. for $\left[\mathrm{C}_{12} \mathrm{H}_{28} \mathrm{CuN}_{4}\right]^{2+}, 145.5799$, found 145.5803 $[\mathrm{Cu}(\mathrm{DMC})]^{2+}$, calcd. for $\left[\mathrm{C}_{12} \mathrm{H}_{27} \mathrm{CuN}_{4}\right]^{+}, 290.1526$, found $290.1526[\mathrm{Cu}(\mathrm{DMC})-\mathrm{H}]^{+}$, calcd. for $\left[\mathrm{C}_{12} \mathrm{H}_{28} \mathrm{CuClN}_{4}\right]^{+}$, 326.1293, found $326.1292[\mathrm{Cu}(\mathrm{DMC})+\mathrm{Cl}]^{+}$, calcd. for $\left[\mathrm{C}_{13} \mathrm{H}_{29} \mathrm{CuN}_{4} \mathrm{O}_{2}\right]^{+}$, 336.1581, found $336.1581[\mathrm{Cu}(\mathrm{DMC})+\mathrm{HCOO}]^{+}$.

\section{Zinc(II) 1,8-dimethyl-1,4,8,11-tetrazacyclotetradecane dichloride}

DMC (41.1 mg, $0.180 \mathrm{mmol}), \mathrm{ZnCl}_{2}$ (26.9 mg, $\left.0.198 \mathrm{mmol}\right)$.

Complex was obtained as a beige powder (48.2 mg, $73 \%)$.

ESI-HR-MS (positive, $\mathrm{H}_{2} \mathrm{O}$ ): $\mathrm{m} / z$ calcd. for $\left[\mathrm{C}_{12} \mathrm{H}_{28} \mathrm{~N}_{4} \mathrm{Zn}\right]^{2+} 146.0797$, found 146.0800 $[\mathrm{Zn}(\mathrm{DMC})]^{2+}$, calcd. for $\left[\mathrm{C}_{12} \mathrm{H}_{28} \mathrm{ClN}_{4} \mathrm{Zn}\right]^{+}, 327.1289$, found $327.1289[\mathrm{Zn}(\mathrm{DMC})+\mathrm{Cl}]^{+}$, calc. for $\left[\mathrm{C}_{13} \mathrm{H}_{29} \mathrm{~N}_{4} \mathrm{O}_{2} \mathrm{Zn}\right]^{+}, 337.1576$, found $337.1577[\mathrm{Zn}(\mathrm{DMC})+\mathrm{HCOO}]^{+} .{ }^{\mathbf{1}} \mathbf{H} \mathbf{~ N M R}(500 \mathrm{MHz}$, 
$\mathrm{D}_{2} \mathrm{O}, 298 \mathrm{~K}$ ): too complex mixture of 3 isomers (See below). ${ }^{\mathbf{1 3}} \mathbf{C}$ NMR (125 MHz, $\mathrm{D}_{2} \mathrm{O}, 298$ K): $\underline{\text { trans-III: } \delta=[62.3,55.3,52.2,47.9]}\left(\mathrm{CH}_{2}-\alpha-\mathrm{N}\right), 44.7\left(\mathrm{~N}^{-\mathrm{CH}_{3}}\right), 27.0 \mathrm{ppm}\left(\mathrm{CH}_{2}-\beta-\mathrm{N}\right) \cdot \underline{\text { trans- }}$ I: $\delta=[63.1,60.8,54.0,47.7]\left(\mathrm{CH}_{2}-\alpha-\mathrm{N}\right), 42.7\left(\mathrm{CH}_{3}\right), 26.6 \mathrm{ppm}\left(\mathrm{CH}_{2}-\beta-\mathrm{N}\right) . \underline{\text { cis }-\mathrm{V}:} \delta=[62.7$, 59.3, 53.7, 48.3] $\left(\mathrm{CH}_{2}-\alpha-\mathrm{N}\right), 48.3\left(\mathrm{CH}_{3}\right), 26.4 \mathrm{ppm}\left(\mathrm{CH}_{2}-\beta-\mathrm{N}\right)$.

\section{Copper(II) 1,4,8,11-tetramethyl-1,4,8,11-tetrazacyclotetradecane dichloride}

TMC (46.1 mg, $0.180 \mathrm{mmol}), \mathrm{CuCl}_{2}$ (26.6 mg, $\left.0.198 \mathrm{mmol}\right)$.

Complex was obtained as a blue powder $(57.3 \mathrm{mg}, 82 \%)$.

ESI-MS (positive, $\mathrm{H}_{2} \mathrm{O} / \mathrm{CH}_{3} \mathrm{CN} 50 / 50$ ): $\mathrm{m} / \mathrm{z}$ found $159.582[\mathrm{Cu}(\mathrm{TMC})]^{2+}$, found 354.129 $[\mathrm{Cu}(\mathrm{TMC})+\mathrm{Cl}]^{+}$.

\section{Zinc(II) 1,4,8,11-tetramethyl-1,4,8,11-tetrazacyclotetradecane dichloride}

TMC (46.1 mg, $0.180 \mathrm{mmol}), \mathrm{ZnCl}_{2}$ (26.9 mg, $\left.0.198 \mathrm{mmol}\right)$.

Complex was obtained as a white powder (50.9 mg, $72 \%)$.

ESI-MS (positive, $\mathrm{H}_{2} \mathrm{O} / \mathrm{CH}_{3} \mathrm{CN} 50 / 50$ ): $\mathrm{m} / z$ found $160.0806[\mathrm{Zn}(\mathrm{TMC})]^{2+}$, found 355.1299 $[\mathrm{Zn}(\mathrm{TMC})+\mathrm{Cl}]^{+}$, found $365.1583[\mathrm{Zn}(\mathrm{TMC})+\mathrm{HCOO}]^{+} .{ }^{1} \mathbf{H}$ NMR $\left(500 \mathrm{MHz}, \mathrm{CD}_{3} \mathrm{OD}, 223 \mathrm{~K}\right)$ : trans-I: $\delta=3.37-3.31(\mathrm{~m}, 6 \mathrm{H}), 3.02-2.97(\mathrm{~m}, 2 \mathrm{H}), 2.85-2.83(\mathrm{~m}, 2 \mathrm{H}), 2.58\left(\mathrm{~s}, 6 \mathrm{H}, \mathrm{CH}_{3}\right), 2.53$ (s, 6H, $\left.\mathrm{CH}_{3}\right), 2.41-2.34(\mathrm{~m}, 6 \mathrm{H}), 2.28-2.25(\mathrm{~m}, 2 \mathrm{H}), 1.68-1.65 \mathrm{ppm}(\mathrm{m}, 2 \mathrm{H}) .{ }^{13} \mathrm{C}$ NMR (125 $\left.\mathrm{MHz}, \mathrm{CD}_{3} \mathrm{OD}, 223 \mathrm{~K}\right): \underline{\text { trans-I: }} \delta=[64.4,58.2,58.1,56.4]\left(\mathrm{CH}_{2}-\alpha-\mathrm{N}\right),[46.9,44.5]\left(\mathrm{N}_{-} \mathrm{CH}_{3}\right)$, $22.2 \mathrm{ppm}\left(\mathrm{CH}_{2}-\beta-\mathrm{N}\right)$.

\section{Copper(II) 1,4,8,11-tetraazabicyclo[6.6.2]hexadecane dichloride}

CB-cyclam (39 mg, $0.172 \mathrm{mmol}), \mathrm{CuCl}_{2}(25.4 \mathrm{mg}, 0.189 \mathrm{mmol})$.

Complex was obtained as a blue powder (35.1 mg, $54 \%)$.

ESI-HR-MS (positive, $\mathrm{H}_{2} \mathrm{O}$ ): $\mathrm{m} / z$ calcd. for $\left[\mathrm{C}_{12} \mathrm{H}_{26} \mathrm{CuN}_{4}\right]^{2+}, 144.5721$, found 144.5722 $[\mathrm{Cu}(\mathrm{CB}-\mathrm{cyc})]^{2+}$, calcd. for $\left[\mathrm{C}_{12} \mathrm{H}_{26} \mathrm{CuN}_{4} \mathrm{O}\right]^{2+}, 152.5696$, found $152.5694 \quad[\mathrm{Cu}(\mathrm{CB}-$ cyclam $)+\mathrm{O}]^{2+}$, calcd. for $\left[\mathrm{C}_{12} \mathrm{H}_{26} \mathrm{ClCuN}_{4}\right]^{+}, 324.1136$, found $324.1132[\mathrm{Cu}(\mathrm{CB}-\mathrm{cyclam})+\mathrm{Cl}]^{+}$, calcd. for $\left[\mathrm{C}_{13} \mathrm{H}_{27} \mathrm{CuN}_{4} \mathrm{O}_{2}\right]^{+}$, calcd 334.1425, found 334.1421 [Cu(CB-cyclam) $\left.+\mathrm{HCOO}\right]^{+}$.

\section{Zinc(II) 1,4,8,11-tetraazabicyclo[6.6.2]hexadecane dichloride}

CB-cyclam (39 mg, $0.172 \mathrm{mmol}), \mathrm{ZnCl}_{2}(25.8 \mathrm{mg}, 0.189 \mathrm{mmol})$.

Complex was obtained as a beige powder $(40.2 \mathrm{mg}, 62 \%)$.

ESI-HR-MS (positive, $\mathrm{H}_{2} \mathrm{O}$ ): $\mathrm{m} / z$ calcd. for $\left[\mathrm{C}_{12} \mathrm{H}_{26} \mathrm{~N}_{4} \mathrm{Zn}\right]^{2+}, 145.0719$, found 145.0721 $[\mathrm{Zn}(\mathrm{CB}-\mathrm{cyclam})]^{2+}$, calcd. for $\left[\mathrm{C}_{13} \mathrm{H}_{27} \mathrm{~N}_{4} \mathrm{O}_{2} \mathrm{Zn}\right]^{+}$, calcd 335.1419 , found 335.1420 [Zn(CBcyclam)+HCOO] ${ }^{+} .{ }^{1} \mathbf{H}$ NMR $\left(500 \mathrm{MHz}, \mathrm{D}_{2} \mathrm{O}, 298 \mathrm{~K}\right): \underline{c i s-\mathrm{V}:} \delta=3.22-3.14(\mathrm{~m}, 4 \mathrm{H}), 3.05-2.89$ 
(m, 14H), 2.68-2.65 (m, 2H), 2.32-2.24 (m, 2H), 1.60 ppm (dquint, J = 11.7, $2.8 \mathrm{~Hz}, 2 \mathrm{H}) .{ }^{13} \mathbf{C}$ NMR $\left(125 \mathrm{MHz}, \mathrm{D}_{2} \mathrm{O}, 298 \mathrm{~K}\right): \underline{c i s-\mathrm{V}}: \delta=[62.1,60.8,53.5,50.6,43.5]\left(\mathrm{CH}_{2}-\alpha-\mathrm{N}\right), 24.1 \mathrm{ppm}$ $\left(\mathrm{CH}_{2}-\beta-\mathrm{N}\right)$.

\section{Copper(II) 4,11-dimethyl-1,4,8,11-tetraazabicyclo[6.6.2] hexadecane dichloride}

CB-DMC (127 mg, $0.499 \mathrm{mmol}), \mathrm{CuCl}_{2}(67.1 \mathrm{mg}, 0.499 \mathrm{mmol})$.

Complex was obtained as a green powder $(80.0 \mathrm{mg}, 41 \%)$.

ESI-MS (positive, $\mathrm{H}_{2} \mathrm{O} / \mathrm{CH}_{3} \mathrm{CN} 50 / 50$ ): $\mathrm{m} / z$ found $158.575[\mathrm{Cu}(\mathrm{CB}-\mathrm{DMC})]^{2+}$, found 352.113 $[\mathrm{Cu}(\mathrm{CB}-\mathrm{DMC})+\mathrm{Cl}]^{+}$, found $362.139[\mathrm{Cu}(\mathrm{CB}-\mathrm{DMC})+\mathrm{HCOO}]^{+}$.

\section{Zinc(II) 4,11-dimethyl-1,4,8,11-tetraazabicyclo[6.6.2]hexadecane dichloride.}

CB-DMC (80 mg, $0.314 \mathrm{mmol}), \mathrm{CuCl} 2$ (42.7 mg, $0.314 \mathrm{mmol})$.

Complex was obtained as a white powder $(60.2 \mathrm{mg}, 50 \%)$.

ESI-HR-MS (positive, $\mathrm{H}_{2} \mathrm{O}$ ): $\mathrm{m} / z$ calcd. for $\left[\mathrm{C}_{14} \mathrm{H}_{30} \mathrm{~N}_{4} \mathrm{Zn}\right]^{2+}, 159.0879$, found 159.0875 $[\mathrm{Zn}(\mathrm{CB}-\mathrm{DMC})]^{2+}$, calcd. for $\left[\mathrm{C}_{14} \mathrm{H}_{30} \mathrm{ClN}_{4} \mathrm{Zn}\right]^{+}$, 353.1445, found 353.1445 [Zn(CB$\mathrm{DMC})+\mathrm{Cl}]^{+}$, calcd. for $\left[\mathrm{C}_{15} \mathrm{H}_{31} \mathrm{~N}_{4} \mathrm{O}_{2} \mathrm{Zn}\right]^{+}, 363.1734$, found $363.1733[\mathrm{Zn}(\mathrm{CB}-\mathrm{DMC})+\mathrm{HCOO}]^{+}$. ${ }^{1} \mathbf{H}$ NMR (500 MHz, CD $\left.{ }_{3} \mathrm{OD}, 223 \mathrm{~K}\right): \underline{c i s-\mathrm{V}:} \delta=3.39-3.32(\mathrm{~m}, 2 \mathrm{H}), 3.18(\mathrm{dt}, \mathrm{J}=15.6,3 \mathrm{~Hz}$, 2H), 3.00-2.95 (m, 4H), 2.85-2.83 (m, 4H), 2.57-2.50 (m, 6H), 2.26 (s, 6H, CH3), 2.27-2.22 (m, 2H), 2.09 (dd, J = 14.5, $3 \mathrm{~Hz}, 2 \mathrm{H}), 1.54$ (dt, J = 17, $3 \mathrm{~Hz}, 2 \mathrm{H}) .{ }^{13} \mathbf{C}$ NMR (125 MHz, $\mathrm{CD}_{3} \mathrm{OD}$, $223 \mathrm{~K}): \underline{c i s-\mathrm{V}:} \delta=[61.0,60.7,60.7,52.7,52.5]\left(\mathrm{CH}_{2}-\alpha-\mathrm{N}\right), 49.1\left(\mathrm{~N}_{-} \mathrm{CH}_{3}\right), 24.7 \mathrm{ppm}\left(\mathrm{CH}_{2}-\beta-\right.$ N).

\section{Copper(II) 2-(1,4,8,11-tetrazacyclotetradec-6-yl)ethanol dichloride}

Cyclam-E (44.0 mg, $0.180 \mathrm{mmol}), \mathrm{CuCl}_{2}(26.6 \mathrm{mg}, 0.198 \mathrm{mmol})$.

Complex was obtained as a pink powder $(50.6 \mathrm{mg}, 78 \%)$.

ESI-HR-MS (positive, $\mathrm{H}_{2} \mathrm{O}$ ): $\mathrm{m} / z$ calcd. for $\left[\mathrm{C}_{12} \mathrm{H}_{28} \mathrm{CuN}_{4} \mathrm{O}\right]^{2+}, 153.5774$, found 153.5777 $[\mathrm{Cu}(\text { cyclam-E })]^{2+}$, calcd. for $\left[\mathrm{C}_{12} \mathrm{H}_{27} \mathrm{CuN}_{4} \mathrm{O}\right]^{+}, 306.1465$, found $342.1471\left[\mathrm{Cu}(\text { cyclam-E)-H}]^{+}\right.$, calcd. for $\left[\mathrm{C}_{12} \mathrm{H}_{28} \mathrm{ClCuN}_{4} \mathrm{O}\right]^{+}, 342.1242$, found $342.1234\left[\mathrm{Cu}(\text { cyclam-E) }+\mathrm{Cl}]^{+}\right.$.

\section{Zinc(II) 2-(1,4,8,11-tetrazacyclotetradec-6-yl)ethanol dichloride}

Cyclam-E (44.0 mg, $0.180 \mathrm{mmol}), \mathrm{CuCl}_{2}(26.9 \mathrm{mg}, 0.198 \mathrm{mmol})$.

Complex was obtained as a beige powder $(47.6 \mathrm{mg}, 69 \%)$.

ESI-HR-MS (positive, $\mathrm{H}_{2} \mathrm{O}$ ): $\mathrm{m} / z$ calcd. for $\left[\mathrm{C}_{12} \mathrm{H}_{28} \mathrm{~N}_{4} \mathrm{OZn}\right]^{2+}, 154.0772$, found 154.0776 $\left[\mathrm{Zn}(\text { cyclam-E) }]^{2+}\right.$, calcd. for $\left[\mathrm{C}_{12} \mathrm{H}_{28} \mathrm{ClN}_{4} \mathrm{OZn}\right]^{+}, 343.1238$, found 343.1237 [Zn(cyclamE) $+\mathrm{Cl}]^{+}$, calcd. for $\left[\mathrm{C}_{13} \mathrm{H}_{29} \mathrm{~N}_{4} \mathrm{O}_{3} \mathrm{Zn}\right]^{+}, 353.1526$, found 353.1527 [Zn(cyclam-E) $\left.+\mathrm{HCOO}\right]^{+} .{ }^{1} \mathbf{H}$ 
NMR (500 MHz, D $2 \mathrm{O}, 298 \mathrm{~K})$ : trans-III: $\delta=3.71\left(\mathrm{t}, \mathrm{J}=6.7 \mathrm{~Hz}, 2 \mathrm{H}, \mathrm{CH}_{2}-\alpha-\mathrm{OH}\right), 3.26-3.19$ (m, 3H), 3.15-2.98 (m, 6H), 2.84-2.76 (m, 4H), 2.61-2.57 (m, 3H), 1.97-1.94 (m, 2H, CH- $\beta-\mathrm{N}$ $\left.\left.+\mathrm{CH}_{2}-\beta-\mathrm{N}\right), 1.76-1.71\left(\mathrm{~m}, 1 \mathrm{H}, \mathrm{CH}_{2}-\beta-\mathrm{N}\right)\right), 1.59-1.55 \mathrm{ppm}\left(\mathrm{m}, 2 \mathrm{H}, \mathrm{CH}_{2}-\beta-\mathrm{OH}\right) .{ }^{13} \mathbf{C}$ NMR (125 $\left.\mathrm{MHz}, \mathrm{D}_{2} \mathrm{O}, 298 \mathrm{~K}\right)$ : trans-III: $\delta=62.0\left(\mathrm{CH}_{2}-\alpha-\mathrm{OH}\right),[57.9,52.8,50.4,50.4]\left(\mathrm{CH}_{2}-\alpha-\mathrm{N}\right), 37.9$ $\left(\mathrm{CH}_{2}-\beta-\mathrm{OH}\right), 36.8(\mathrm{CH}-\beta-\mathrm{N}), 30.7 \mathrm{ppm}\left(\mathrm{CH}_{2}-\beta-\mathrm{N}\right)$. trans-I and $\underline{c i s-\mathrm{V}}$ are unidentifiable (See below).

\section{Copper(II) 2-(1,8-dimethyl-1,4,8,11-tetrazacyclotetradec-6-yl)ethanol dichloride}

DMC-E (49.0 mg, $0.180 \mathrm{mmol}), \mathrm{CuCl}_{2}(26.6 \mathrm{mg}, 0.198 \mathrm{mmol})$.

Complex was obtained as a purple powder $(58.0 \mathrm{mg}, 79 \%)$.

ESI-HR-MS (positive, $\mathrm{H}_{2} \mathrm{O}$ ): $\mathrm{m} / z$ calcd. for $\left[\mathrm{C}_{14} \mathrm{H}_{32} \mathrm{CuN}_{4} \mathrm{O}\right]^{2+}, 167.5931$, found 167.5934 $[\mathrm{Cu}(\mathrm{DMC}-\mathrm{E})]^{2+}$, calcd. for $\left[\mathrm{C}_{14} \mathrm{H}_{31} \mathrm{CuN}_{4} \mathrm{O}\right]^{+}, 334.1788$, found 334.1784 [Cu(DMC-E)-H] $]^{+}$, calcd. for $\left[\mathrm{C}_{14} \mathrm{H}_{32} \mathrm{ClCuN}_{4} \mathrm{O}\right]^{+}, 370.1555$, found 370.1554 [Cu(DMC-E)+Cl] .

\section{Zinc(II) 2-(1,8-dimethyl-1,4,8,11-tetrazacyclotetradec-6-yl)ethanol dichloride}

DMC-E (49.0 mg, $0.180 \mathrm{mmol}), \mathrm{ZnCl}_{2}(26.9 \mathrm{mg}, 0.198 \mathrm{mmol})$.

Complex was obtained as a beige powder $(53.8 \mathrm{mg}, 73 \%)$.

ESI-HR-MS (positive, $\mathrm{H}_{2} \mathrm{O}$ ): $\mathrm{m} / z$ calcd. for $\left[\mathrm{C}_{14} \mathrm{H}_{32} \mathrm{~N}_{4} \mathrm{OZn}\right]^{2+}, 168.0928$, found 168.0931 $[\mathrm{Zn}(\mathrm{DMC}-\mathrm{E})]^{2+},\left[\mathrm{C}_{15} \mathrm{H}_{33} \mathrm{~N}_{4} \mathrm{O}_{3} \mathrm{Zn}\right]^{+}, 381.1839$, found $381.1837[\mathrm{Zn}(\mathrm{DMC}-\mathrm{E})+\mathrm{HCOO}]^{+} .{ }^{1} \mathbf{H}$ NMR (500 MHz, $\left.\mathrm{D}_{2} \mathrm{O}, 298 \mathrm{~K}\right)$ : too complex mixture of 3 isomers (See below). ${ }^{13} \mathbf{C}$ NMR (125 $\left.\mathrm{MHz}, \mathrm{D}_{2} \mathrm{O}, 298 \mathrm{~K}\right)$ : isomers trans-III + cis-V: $\delta=[68.5,68.3,63.0,62.5,61.8,61.7,59.6,59.4$, 59.0, 57.5, 56.2, 55.3, 53.7, 52.3, 48.6, 48.5, 48.1, 57.9] $\left(\mathrm{CH}_{2}-\alpha-\mathrm{N}, \mathrm{CH}_{2}-\alpha-\mathrm{OH}\right)$, [48.6, 48.5, 45.9, 45.4] (N-CH3), [36.9, 36.6] $\left(\mathrm{CH}_{2}-\beta-\mathrm{OH}\right),[33.8,33.2](\mathrm{CH}-\beta-\mathrm{N}),[27.2,26.6] \mathrm{ppm}$ $\left(\mathrm{CH}_{2}-\beta-\mathrm{N}\right)$. trans-I isomer are unidentifiable (See below).

\section{Copper(II) 2-(1,4,8,11-tetramethyl-1,4,8,11-tetrazacyclotetradec-6-yl)ethanol dichloride}

TMC-E (54.1 mg, $0.180 \mathrm{mmol}), \mathrm{CuCl}_{2}(26.6 \mathrm{mg}, 0.198 \mathrm{mmol})$.

Complex was obtained as a blue powder $(60.2 \mathrm{mg}, 77 \%)$.

ESI-HR-MS (positive, $\mathrm{H}_{2} \mathrm{O}$ ): $\mathrm{m} / z$ calcd. for $\left[\mathrm{C}_{16} \mathrm{H}_{36} \mathrm{CuN}_{4} \mathrm{O}\right]^{2+}, 181.6087$, found 181.6092 $[\mathrm{Cu}(\mathrm{TMC}-\mathrm{E})]^{2+}$, calcd. for $\left[\mathrm{C}_{16} \mathrm{H}_{36} \mathrm{CuN}_{4} \mathrm{O}\right]^{+}, 363.2180$, found $363.2179[\mathrm{Cu}(\mathrm{TMC}-\mathrm{E})]^{+}$, $\left[\mathrm{C}_{16} \mathrm{H}_{36} \mathrm{ClCuN}_{4} \mathrm{O}\right]^{+}, 398.1868$, found $398.1867[\mathrm{Cu}(\mathrm{TMC}-\mathrm{E})+\mathrm{Cl}]^{+}$.

\section{Zinc(II) 2-(1,4,8,11-tetramethyl-1,4,8,11-tetrazacyclotetradec-6-yl)ethanol dichloride} TMC-E (54.1 mg, $0.180 \mathrm{mmol}), \mathrm{ZnCl}_{2}$ (26.9 mg, $\left.0.198 \mathrm{mmol}\right)$.

Complex was obtained as a white powder $(54.3 \mathrm{mg}, 69 \%)$. 
ESI-HR-MS (positive, $\mathrm{H}_{2} \mathrm{O}$ ): $\mathrm{m} / z$ calcd. for $\left[\mathrm{C}_{14} \mathrm{H}_{32} \mathrm{~N}_{4} \mathrm{OZn}\right]^{2+}, 168.0928$, found 168.0931 $[\mathrm{Zn}(\mathrm{TMC}-\mathrm{E})]^{2+}$, calcd. for $\left[\mathrm{C}_{17} \mathrm{H}_{36} \mathrm{ClN}_{4} \mathrm{OZn}\right]^{+}, 399.1864$, found 399.1869 [Zn(TMC-E)+Cl] $]^{+}$, calcd. for $\left[\mathrm{C}_{17} \mathrm{H}_{37} \mathrm{~N}_{4} \mathrm{O}_{3} \mathrm{Zn}\right]^{+}, 409.2152$, found 409.2151 [Zn(TMC-E)+HCOO] ${ }^{+}{ }^{1} \mathbf{H}$ NMR (500 $\left.\mathrm{MHz}, \mathrm{CD}_{3} \mathrm{OD}, 223 \mathrm{~K}\right)$ : trans-I: $\delta=3.66\left(\mathrm{~m}, 2 \mathrm{H}, \mathrm{CH}_{2}-\alpha-\mathrm{OH}\right), 3.38-3.31(\mathrm{~m}, 4 \mathrm{H}), 3.18-3.14(\mathrm{~m}$, $2 \mathrm{H}), 3.02-2.97(\mathrm{~m}, 2 \mathrm{H}), 2.84-2.82(\mathrm{~m}, 1 \mathrm{H}), 2.76-2.73(\mathrm{~m}, 1 \mathrm{H}),[2.60,2.58,2.54,2.53](4 \mathrm{~s}, 12 \mathrm{H}$, $\mathrm{CH}_{3}$ ), 2.53-2.49 (m, 1H, CH- $\left.\beta-\mathrm{N}\right), 2.40-2.33$ (m, 4H), 2.28-2.25 (m, 3H), 1.67-1.64 (m, 1H, $\left.\mathrm{CH}_{2}-\beta-\mathrm{N}\right), 1.48-1.47 \mathrm{ppm}\left(\mathrm{m}, 2 \mathrm{H}, \mathrm{CH}_{2}-\beta-\mathrm{OH}\right) .{ }^{13} \mathbf{C}$ NMR (125 MHz, $\left.\mathrm{CD}_{3} \mathrm{OD}, 223 \mathrm{~K}\right)$ : trans-I: $\delta=59.7\left(\mathrm{CH}_{2}-\alpha-\mathrm{OH}\right),[69.7,64.3,63.8,58.3,58.1,58.1,56.7,56.3]\left(\mathrm{CH}_{2}-\alpha-\mathrm{N}\right)$, [47.6, 46.9, 45.2, 44.5] $\left(\mathrm{N}-\mathrm{CH}_{3}\right), 35.7\left(\mathrm{CH}_{2}-\beta-\mathrm{OH}\right), 27.8(\mathrm{CH}-\beta-\mathrm{N}), 22.2 \mathrm{ppm}\left(\mathrm{CH}_{2}-\beta-\mathrm{N}\right)$.

\section{Copper(II) 2-(1,4,8,11-tetraazabicyclo[6.6.2]hexadec-6-yl)ethanol dichloride}

CB-cyclam-E (56.0 mg, $0.207 \mathrm{mmol}), \mathrm{CuCl}_{2}(30.5 \mathrm{mg}, 0.227 \mathrm{mmol})$.

Complex was obtained as a blue powder $(44.9,52 \%)$.

ESI-HR-MS (positive, $\mathrm{H}_{2} \mathrm{O}$ ): $\mathrm{m} / z$ calcd. for $\left[\mathrm{C}_{14} \mathrm{H}_{30} \mathrm{CuN}_{4} \mathrm{O}\right]^{2+}, 166.5852$, found 166.5857 $[\mathrm{Cu}(\mathrm{CB}-\mathrm{cyc}-\mathrm{E})]^{2+}$, calcd. for $\left[\mathrm{C}_{14} \mathrm{H}_{30} \mathrm{ClCuN}_{4} \mathrm{O}\right]^{+}, 368.1399$, found $368.1392[\mathrm{Cu}(\mathrm{CB}-\mathrm{cyclam}-$ E) $+\mathrm{Cl}]^{+}$, calcd. for $\left[\mathrm{C}_{15} \mathrm{H}_{31} \mathrm{CuN}_{4} \mathrm{O}_{3}\right]^{+}, 378.1687$, found $378.1682[\mathrm{Cu}(\mathrm{CB}-\text { cyclam-E })+\mathrm{HCOO}]^{+}$.

\section{Zinc(II) 2-(1,4,8,11-tetraazabicyclo[6.6.2]hexadec-6-yl)ethanol dichloride}

CB-cyclam-E (56.0 mg, $0.207 \mathrm{mmol}), \mathrm{ZnCl}_{2}(31.0 \mathrm{mg}, 0.228 \mathrm{mmol})$.

Complex was obtained as beige powder $(50.1 \mathrm{mg}, 57 \%)$.

ESI-HR-MS (positive, $\mathrm{H}_{2} \mathrm{O}$ ): $\mathrm{m} / z$ calcd. for $\left[\mathrm{C}_{14} \mathrm{H}_{30} \mathrm{~N}_{4} \mathrm{OZn}\right]^{2+}, 167.0852$, found 167.0850 $[\mathrm{Zn}(\mathrm{CB}-\mathrm{cyclam}-\mathrm{E})]^{2+}$, calcd. for $\left[\mathrm{C}_{14} \mathrm{H}_{30} \mathrm{ClN}_{4} \mathrm{OZn}\right]^{+}$, 369.1394, found 369.1397 [Zn(CBcyclam-E)+Cl] $]^{+}$, calcd for $\left[\mathrm{C}_{15} \mathrm{H}_{31} \mathrm{~N}_{4} \mathrm{O}_{3} \mathrm{Zn}\right]^{+}, 379.1682$, found 379.1682 [Zn(CB-cyclam$\mathrm{E})+\mathrm{HCOO}]^{+} .{ }^{1} \mathbf{H}$ NMR $\left(500 \mathrm{MHz}, \mathrm{D}_{2} \mathrm{O}, 298 \mathrm{~K}\right)$ : too complex mixture of cis-V syn and cis-V anti isomers (See below). ${ }^{13} \mathrm{C}$ NMR $\left(125 \mathrm{MHz}, \mathrm{D}_{2} \mathrm{O}, 298 \mathrm{~K}\right)$ : cis-V syn: $\delta=63.2\left(\mathrm{CH}_{2}-\alpha-\mathrm{OH}\right)$, $[61.6,61.2,58.6,57.4,55.9,55.2,52.3,51.6,51.3,44.8]\left(\mathrm{CH}_{2}-\alpha-\mathrm{N}\right), 36.3\left(\mathrm{CH}_{2}-\beta-\mathrm{OH}\right), 35.0$

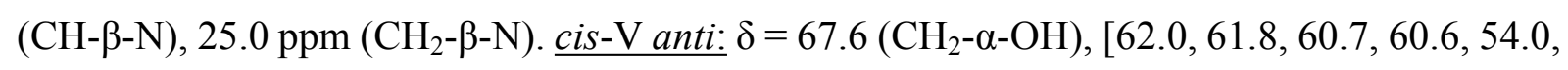
53.3, X, 50.7, 44.4, 43.5] $\left(\mathrm{CH}_{2}-\alpha-\mathrm{N}\right), 36.8\left(\mathrm{CH}_{2}-\beta-\mathrm{OH}\right), 30.2(\mathrm{CH}-\beta-\mathrm{N}), 24.0 \mathrm{ppm}\left(\mathrm{CH}_{2}-\beta-\mathrm{N}\right)$.

Copper(II) 2-(4,11-dimethyl-1,4,8,11-tetraazabicyclo[6.6.2]hexadec-6-yl)ethanol dichloride

CB-DMC-E (60 mg, $0.200 \mathrm{mmol}), \mathrm{CuCl}_{2}(26.9 \mathrm{mg}, 0.200 \mathrm{mmol})$.

Complex was obtained as a green powder $(41.5 \mathrm{mg}, 48 \%)$. 
ESI-HR-MS (positive, $\mathrm{H}_{2} \mathrm{O}$ ): $\mathrm{m} / z$ calcd. for $\left[\mathrm{C}_{16} \mathrm{H}_{34} \mathrm{CuN}_{4} \mathrm{O}\right]^{2+}, 180.6009$, found 180.6013 $[\mathrm{Cu}(\mathrm{CB}-\mathrm{DMC}-\mathrm{E})]^{2+}$, calcd. for $\left[\mathrm{C}_{16} \mathrm{H}_{34} \mathrm{CuN}_{4} \mathrm{O}_{3}\right]^{2+}$, 196.5958, found 196.5962 $[\mathrm{Cu}(\mathrm{CB}-\mathrm{DMC}-\mathrm{E})+2 \mathrm{O}]^{2+}$, calcd. for $\left[\mathrm{C}_{16} \mathrm{H}_{33} \mathrm{CuN}_{4} \mathrm{O}\right]^{+}, \quad 360.1945$, found 360.1948 $[\mathrm{Cu}(\mathrm{CB}-\mathrm{DMC}-\mathrm{E})-\mathrm{H}]^{+}$, calcd. for $\left[\mathrm{C}_{16} \mathrm{H}_{34} \mathrm{ClCuN}_{4} \mathrm{O}\right]^{+}, \quad 396.1712$, found 396.1713 $[\mathrm{Cu}(\mathrm{CB}-\mathrm{DMC}-\mathrm{E})+\mathrm{Cl}]^{+}$.

\section{Zinc(II) 2-(4,11-dimethyl-1,4,8,11-tetraazabicyclo[6.6.2]hexadec-6-yl)ethanol dichloride}

CB-DMC-E (90 mg, $0.301 \mathrm{mmol}), \mathrm{ZnCl}_{2}(41.0 \mathrm{mg}, 0.301 \mathrm{mmol})$.

Complex was obtained as beige powder $(60.2 \mathrm{mg}, 46 \%)$.

ESI-HR-MS (positive, $\mathrm{H}_{2} \mathrm{O}$ ): $\mathrm{m} / z$ calcd. for $\left[\mathrm{C}_{16} \mathrm{H}_{34} \mathrm{~N}_{4} \mathrm{OZn}\right]^{+}, 181.1009$, found 181.1007 $[\mathrm{Zn}(\mathrm{CB}-\mathrm{DMC}-\mathrm{E})]^{2+}$, calcd. for $\left[\mathrm{C}_{16} \mathrm{H}_{34} \mathrm{ClN}_{4} \mathrm{OZn}\right]^{+}, 397.1712$, found 397.1707 [Zn(CB-DMCE) $+\mathrm{Cl}]^{+}$, calcd. for $\left[\mathrm{C}_{17} \mathrm{H}_{35} \mathrm{~N}_{4} \mathrm{O}_{3} \mathrm{Zn}\right]^{+}$, 407.1994, found 407.1995 [Zn(CB-DMC-E)+HCOO $]^{+}$. cis-V anti: ${ }^{1} \mathbf{H}$ NMR $\left(500 \mathrm{MHz}, \mathrm{D}_{2} \mathrm{O}, 298 \mathrm{~K}\right): \delta=3.68(\mathrm{t}, \mathrm{J}=6.7,2 \mathrm{H}), 3.45-3.39(\mathrm{~m}, 2 \mathrm{H}), 3.35-$ $3.29(\mathrm{~m}, 2 \mathrm{H}), 3.15-3.04(\mathrm{~m}, 4 \mathrm{H}), 2.98-2.88(\mathrm{~m}, 4 \mathrm{H}), 2.80-2.71(\mathrm{~m}, 3 \mathrm{H}), 2.64-2.62(\mathrm{~m}, 3 \mathrm{H})$, 2.53-2.49 (m, 1H, CH- $\beta-\mathrm{N}), 2.41$ (s, 3H, $\left.\mathrm{CH}_{3}\right), 2.40\left(\mathrm{~s}, 3 \mathrm{H}, \mathrm{CH}_{3}\right), 2.39-2.34\left(\mathrm{~m}, 1 \mathrm{H}, \mathrm{CH}_{2}-\beta-\mathrm{N}\right)$, $2.30(\mathrm{dd}, \mathrm{J}=14.5,3.1 \mathrm{~Hz}, 1 \mathrm{H}), 2.24(\mathrm{dd}, \mathrm{J}=14.7,3.1 \mathrm{~Hz}, 1 \mathrm{H}) 1.69-1.66\left(\mathrm{~m}, 1 \mathrm{H}, \mathrm{CH}_{2}-\beta-\mathrm{N}\right)$, 1.52-1.48 ppm (m, 2H, CH $\left.2-\beta-O H) .{ }^{13} \mathbf{C ~ N M R ~ ( 1 2 5 ~ M H z , ~} \mathrm{D}_{2} \mathrm{O}, 298 \mathrm{~K}\right): \delta=61.8\left(\mathrm{CH}_{2}-\alpha-\mathrm{OH}\right)$, $[66.9,66.2,61.1,60.8,60.6,60.6,53.7,53.6,52.7,52.7]\left(\mathrm{CH}_{2}-\alpha-\mathrm{N}\right),[49.9,49.7]\left(\mathrm{N}_{-} \mathrm{CH}_{3}\right), 36.8$ $\left(\mathrm{CH}_{2}-\beta-\mathrm{OH}\right), 30.9(\mathrm{CH}-\beta-\mathrm{N}), 24.8 \mathrm{ppm}\left(\mathrm{CH}_{2}-\beta-\mathrm{N}\right) . \underline{c i s-\mathrm{V} \text { syn: }}{ }^{1} \mathbf{H}$ NMR $\left(500 \mathrm{MHz}, \mathrm{D}_{2} \mathrm{O}, 298\right.$ $\mathrm{K}): \delta=3.70(\mathrm{t}, \mathrm{J}=6.7 \mathrm{~Hz}, 2 \mathrm{H}), 3.66-3.59(\mathrm{~m}, 1 \mathrm{H}), 3.50-3.42(\mathrm{~m}, 2 \mathrm{H}), 3.33-3.28(\mathrm{~m}, 1 \mathrm{H}), 3.25-$ $3.20(\mathrm{~m}, 3 \mathrm{H}), 3.17-3.07(\mathrm{~m}, 3 \mathrm{H}), 3.04-2.99(\mathrm{~m}, 2 \mathrm{H}), 2.96-2.93(\mathrm{~m}, 2 \mathrm{H}), 2.90-2.86(\mathrm{~m}, 2 \mathrm{H})$, 2.72-2.69 (m, 1H), $2.58\left(\mathrm{~s}, 3 \mathrm{H}, \mathrm{CH}_{3}\right), 6.61-6.51(\mathrm{~m}, 3 \mathrm{H}), 2.44\left(\mathrm{~s}, 3 \mathrm{H}, \mathrm{CH}_{3}\right), 2.47-2.41(\mathrm{~m}, 1 \mathrm{H})$, $2.28(\mathrm{dd}, \mathrm{J}=14.7,3.9 \mathrm{~Hz}, 1 \mathrm{H}) 1.76-1.69(\mathrm{~m}, 1 \mathrm{H}), 1.47 \mathrm{ppm}(\mathrm{dt}, \mathrm{J}=9.7,6.8 \mathrm{~Hz}, 2 \mathrm{H}) .{ }^{13} \mathbf{C} \mathbf{~ N M R}$ (125 MHz, $\left.\mathrm{D}_{2} \mathrm{O}, 298 \mathrm{~K}\right): \delta=61.8\left(\mathrm{CH}_{2}-\alpha-\mathrm{OH}\right),[67.9,63.4,62.4,61.8,61.4,60.6,59.9,59.5$, 53.8, 53.8, 53.0] $\left(\mathrm{CH}_{2}-\alpha-\mathrm{N}\right),[49.9,48.2]\left(\mathrm{N}_{-} \mathrm{CH}_{3}\right), 37.0\left(\mathrm{CH}_{2}-\beta-\mathrm{OH}\right), 34.4(\mathrm{CH}-\beta-\mathrm{N}), 24.9 \mathrm{ppm}$ $\left(\mathrm{CH}_{2}-\beta-\mathrm{N}\right)$. 


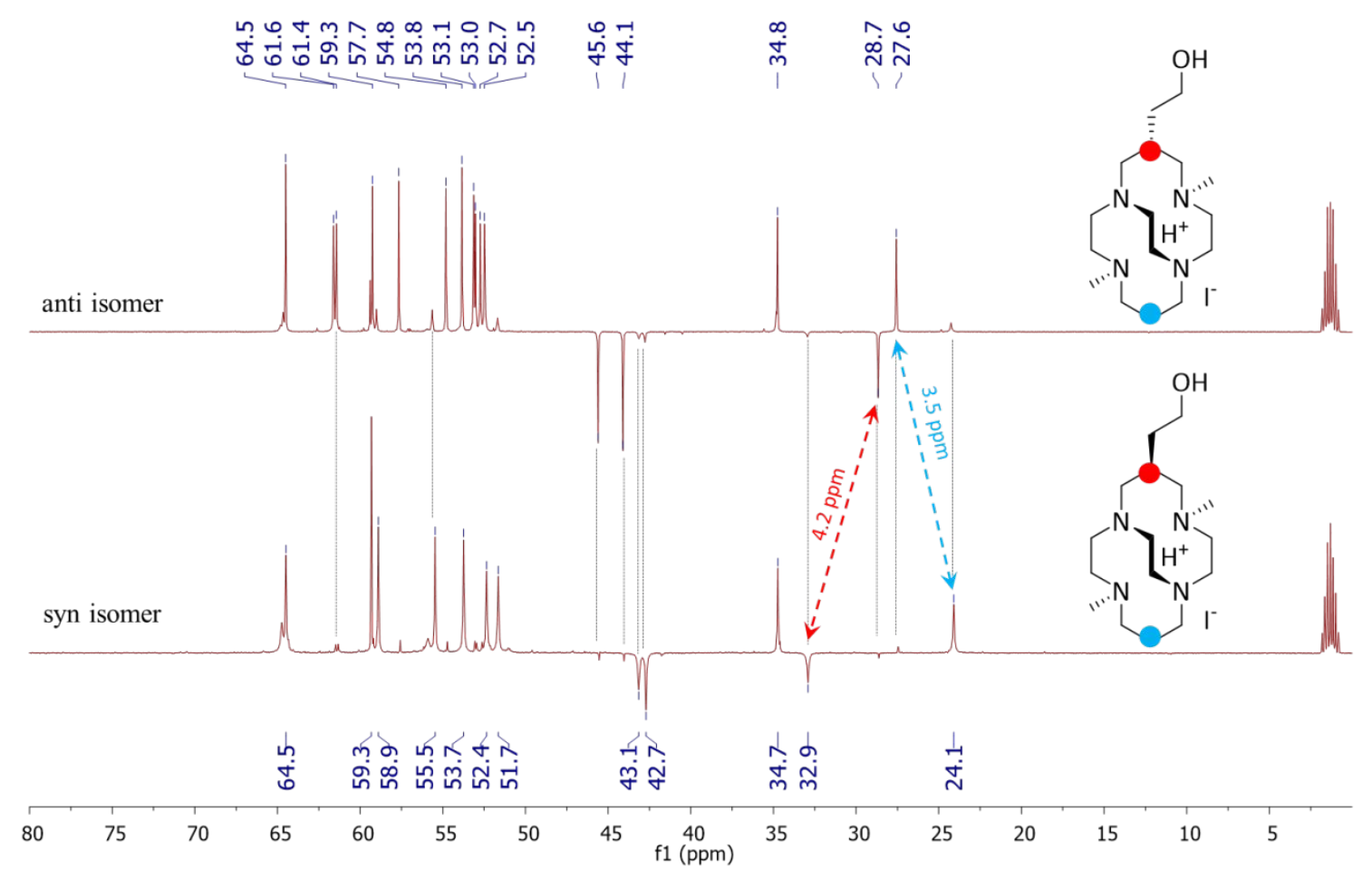

Figure S1. NMR ${ }^{13} \mathrm{C}$ Jmod $\left(125 \mathrm{MHz}, \mathrm{CD}_{3} \mathrm{CN}, 298 \mathrm{~K}\right)$ spectra of anti and syn isomers of CB-DMC-E.

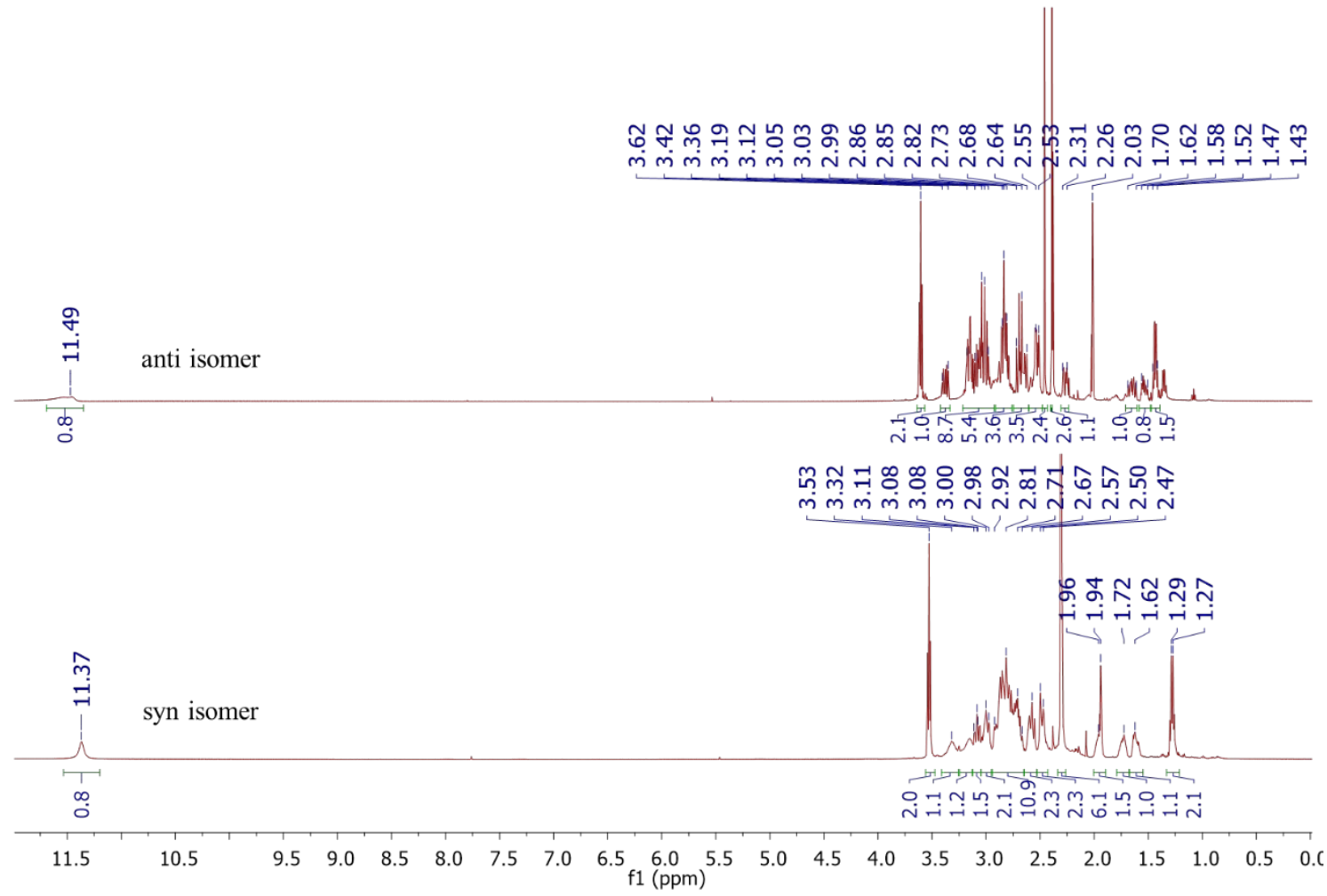

Figure S2. NMR ${ }^{1} \mathrm{H} \mathrm{Jmod}\left(125 \mathrm{MHz}, \mathrm{CD}_{3} \mathrm{CN}, 298 \mathrm{~K}\right)$ spectra of anti and syn isomers of CB-DMC-E. 
. Condition used to obtained crystals discussed in the main manuscript.

Crystals of CB-DMC-E, $[\mathrm{Cu}(\mathbf{D M C}-\mathbf{E}) \mathrm{Cl}] \mathrm{NO}_{3}, \quad[\mathrm{Cu}(\mathbf{T M C}-\mathbf{E}) \mathrm{Cl}]\left(\mathrm{NO}_{3}\right)$ and $[\mathrm{Zn}(\mathbf{T M C}-$ E)Cl] $\left(\mathrm{NO}_{3}\right),\left[\mathrm{Zn}(\mathbf{C B}-\mathbf{c y c l a m}-\mathbf{E}) \mathrm{Cl}_{2}\right]$ syn and anti diastereomers were isolated by diffusion of $\mathrm{Et}_{2} \mathrm{O}$ in a solution of the complex in $\mathrm{CH}_{3} \mathrm{CN}$

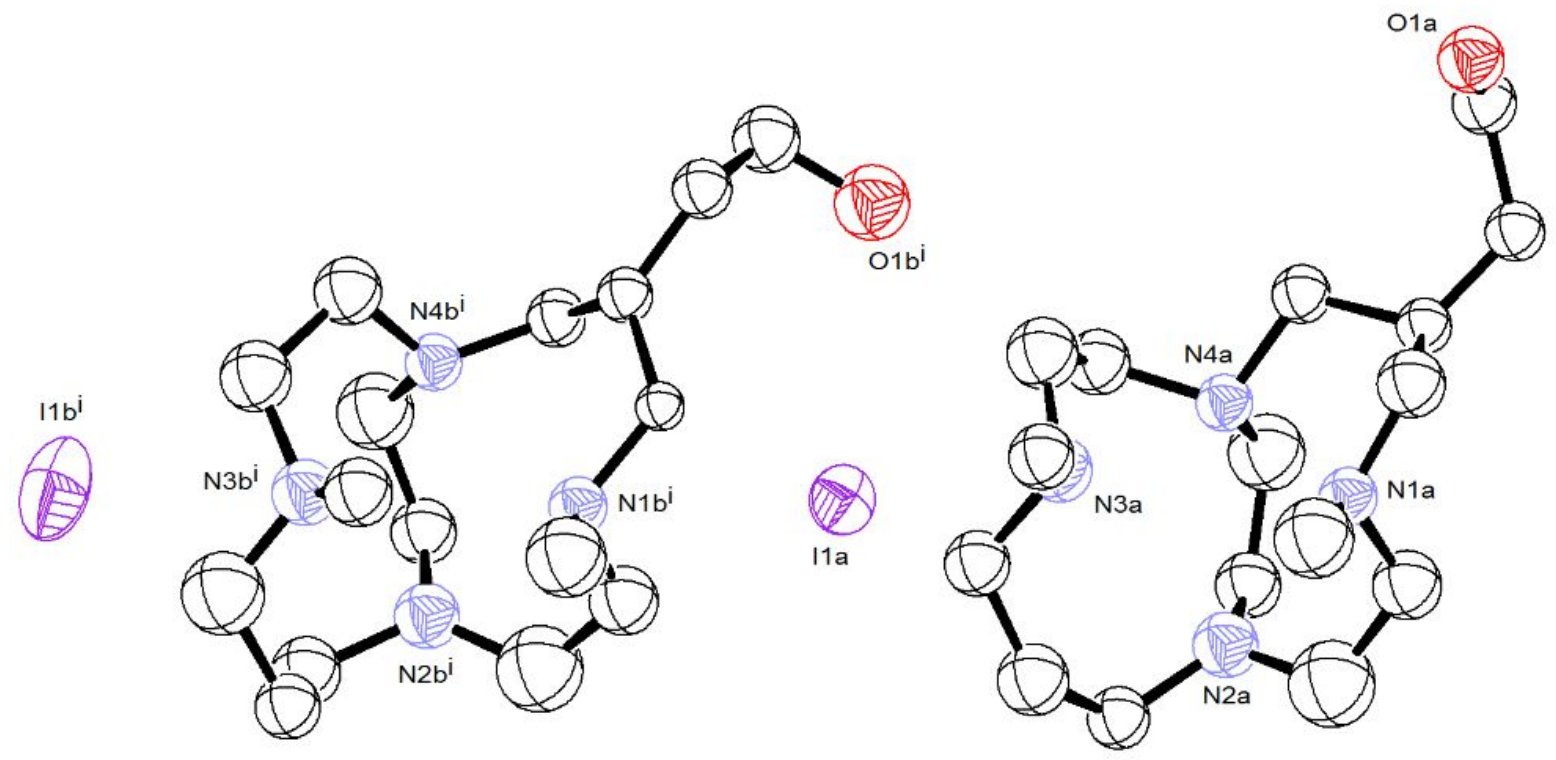

Figure S3. View of the crystal structure of CB-DMC-E showing the two distinct diastereoisomers. Crystals were obtained by slow vapor diffusion of $\mathrm{Et}_{2} \mathrm{O}$ in a solution of a mixture of diastereoisomers in $\mathrm{CH}_{3} \mathrm{CN}$. Hydrogen atoms and water molecules are omitted for clarity. The ORTEP plot is at the $30 \%$ probability level. 
Table S1. Crystallographic data of CB-DMC-E.

\begin{tabular}{ll}
\hline Empirical formula & $\mathrm{C}_{16} \mathrm{H}_{35} \mathrm{IN}_{4} \mathrm{O}$ \\
Formula weight & 426.38 \\
Temperature & $170(2) \mathrm{K}$ \\
Wavelength & $0.71073 \AA$ \\
Crystal system & Monoclinic \\
Space group & $\mathrm{C} 121$ \\
& $\mathrm{a}=15.4401(6) \AA$ \\
Unit cell dimensions & $\mathrm{b}=8.6818(4) \AA \quad \beta=103.363(5)^{\circ}$ \\
& $\mathrm{c}=15.1294(9) \AA$ \\
Volume & $1973.15(17) \AA^{3}$ \\
Z & 4 \\
Density (calculated) & $1.435 \mathrm{mg} / \mathrm{m}^{3}$ \\
Absorption coefficient & $1.631 \mathrm{~mm}{ }^{-1}$ \\
F(000) & 880 \\
Crystal size & $0.30 \times 0.27 \times 0.04 \mathrm{~mm}^{3}$ \\
$\theta$ range for data collection & 3.64 to $26.37 \circ$ \\
Index ranges & $-11<=\mathrm{h}<=19,-10<=\mathrm{k}<=9,-18<=1<=18$ \\
Reflections collected & 6323 \\
Independent reflections & $3697[\mathrm{R}(\mathrm{int})=0.0592]$ \\
Completeness to $\theta=25.242^{\circ}$ & $99.6 \%$ \\
Absorption correction & $\mathrm{Analytical}$ \\
Max. and min. transmission & 0.94 and 0.64 \\
Refinement method & $\mathrm{Full}-\mathrm{matrix}^{\circ} \mathrm{least}-\mathrm{squares}$ on $\mathrm{F}^{2}$ \\
Data/restraints/parameters & $3697 / 570 / 177$ \\
Goodness-of-fit on $\mathrm{F}^{2}$ & 1.043 \\
Final R indices $[\mathrm{I}>2 \mathrm{sigma}(\mathrm{I})]$ & $\mathrm{R} 1=0.0643, \mathrm{wR} 2=0.1611$ \\
$\mathrm{R}$ indices (all data) & $\mathrm{R} 1=0.0863, \mathrm{wR} 2=0.1862$ \\
Extinction coefficient & $\mathrm{n} . \mathrm{a}$. \\
Largest diff. peak and hole & $0.966 \mathrm{and}-0.429 \mathrm{e} \cdot \AA^{-3}$ \\
\hline &
\end{tabular}




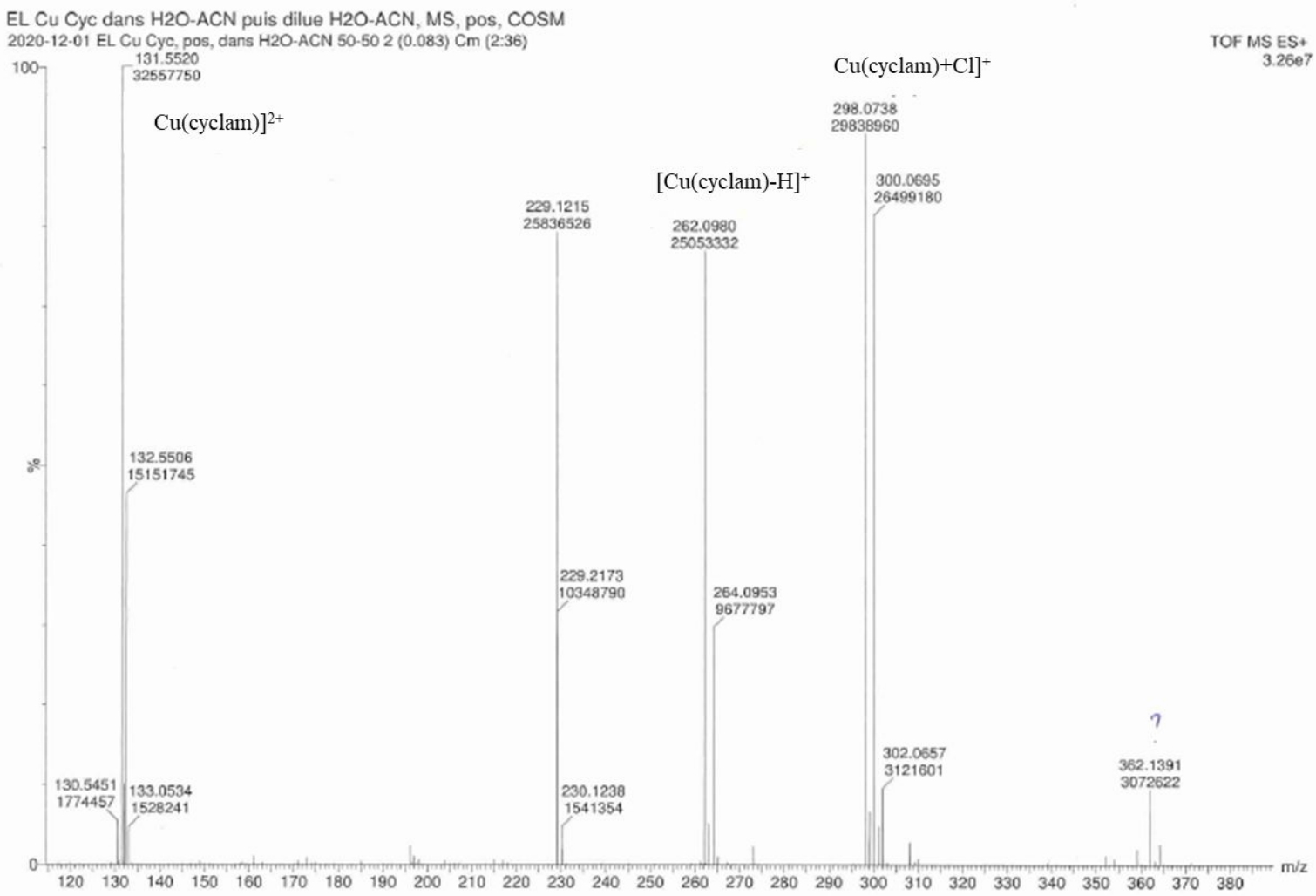

Figure S4. ESI-MS spectrum of $\left[\mathrm{Cu}(\right.$ cyclam $\left.)(\mathrm{Cl})_{2}\right]$.

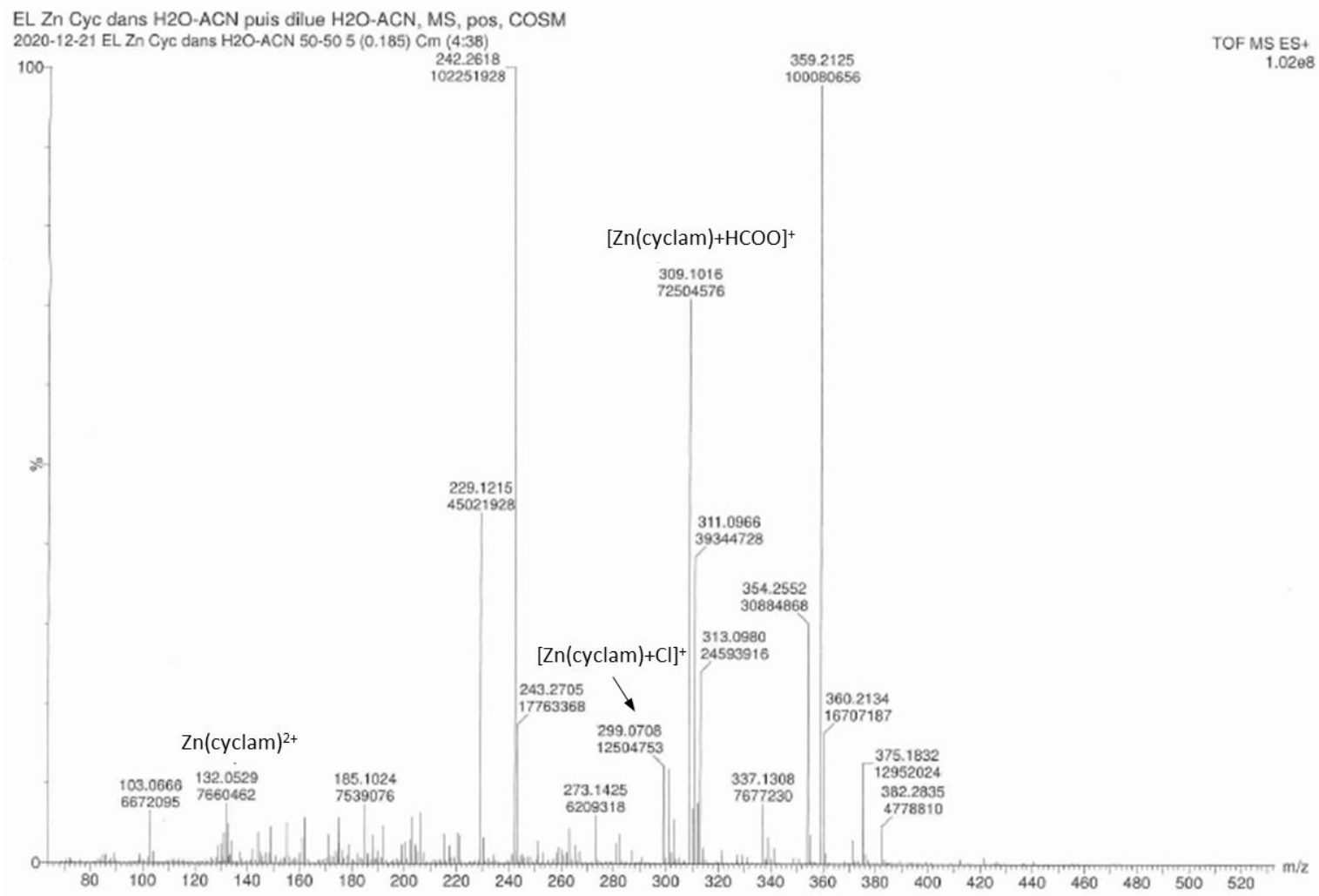

Figure S5. ESI-MS spectrum of $\left[\mathrm{Zn}(\mathbf{c y c l a m})(\mathrm{Cl})_{2}\right]$. 
Fédération de Recherche Physique et Chimie du Vivant (FR2708 : CBM/ICOA) HRAM Plate-forme de Spectrométrie de Masse Haute Résolution

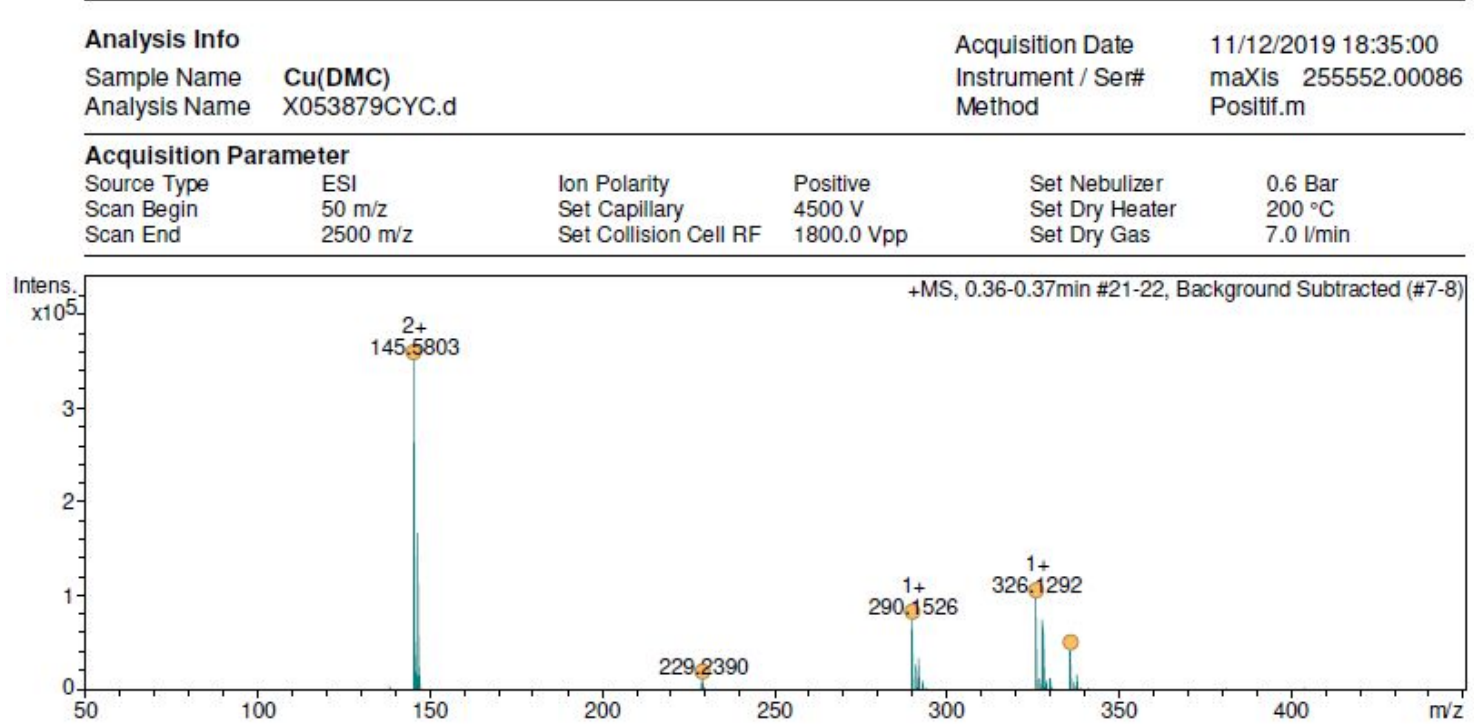

Figure S6. ESI-HRMS spectrum of $\mathrm{Cu}(\mathbf{D M C})^{2+}$.

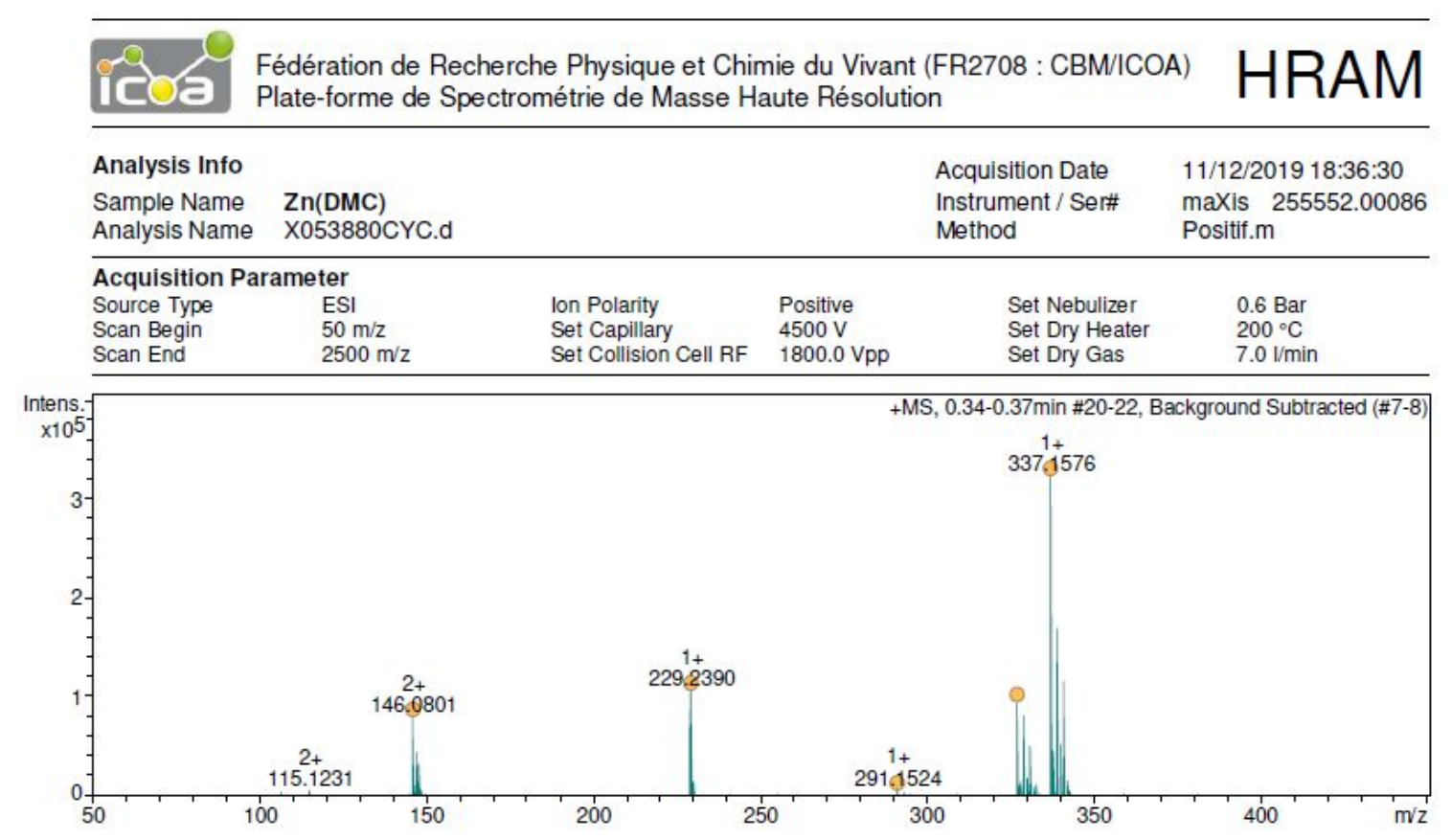

Figure S7. ESI-HRMS spectrum of $\mathrm{Zn}(\mathbf{D M C})^{2+}$. 


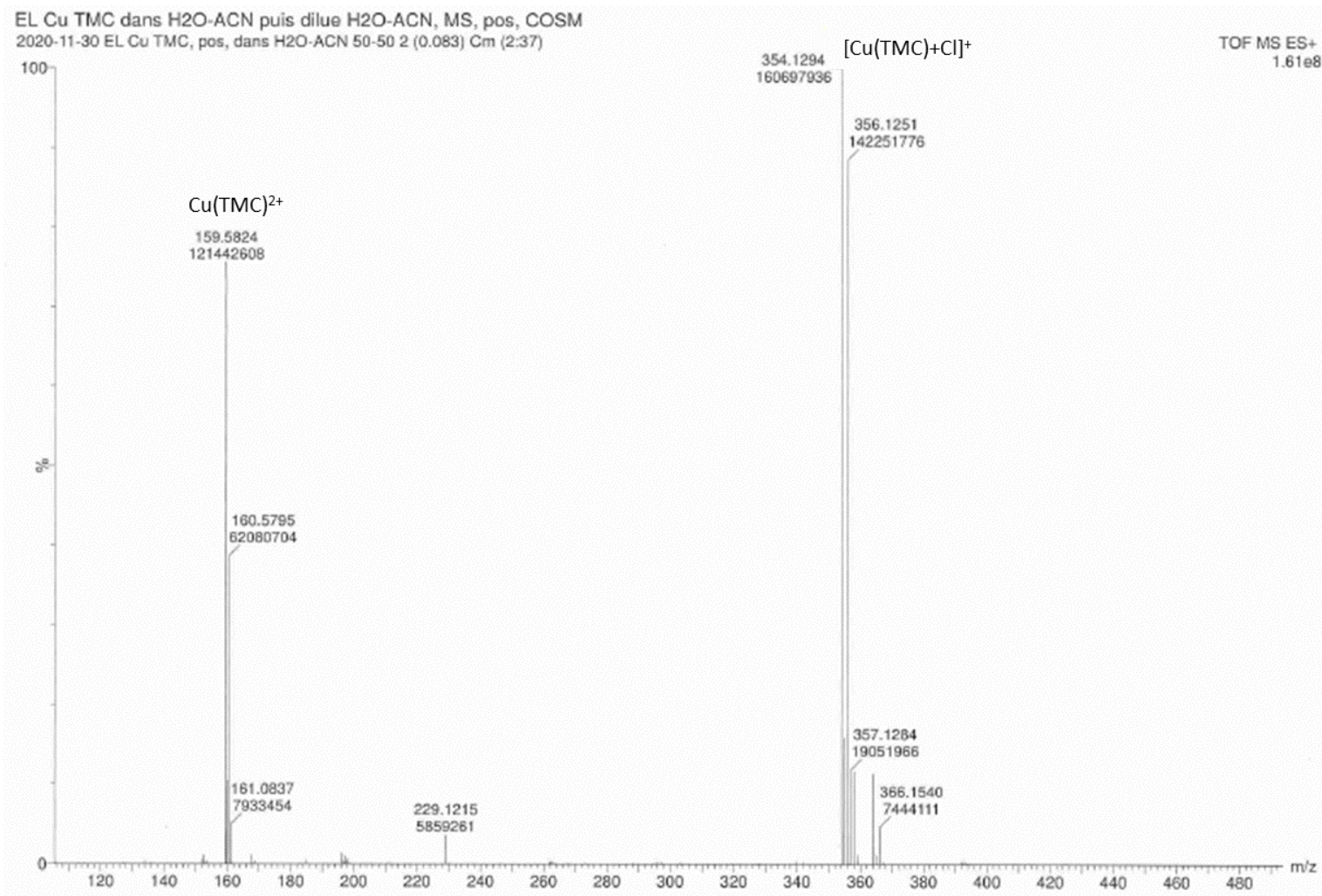

Figure S8. ESI-MS spectrum of $\left[\mathrm{Cu}(\mathbf{T M C})(\mathrm{Cl})_{2}\right]$.

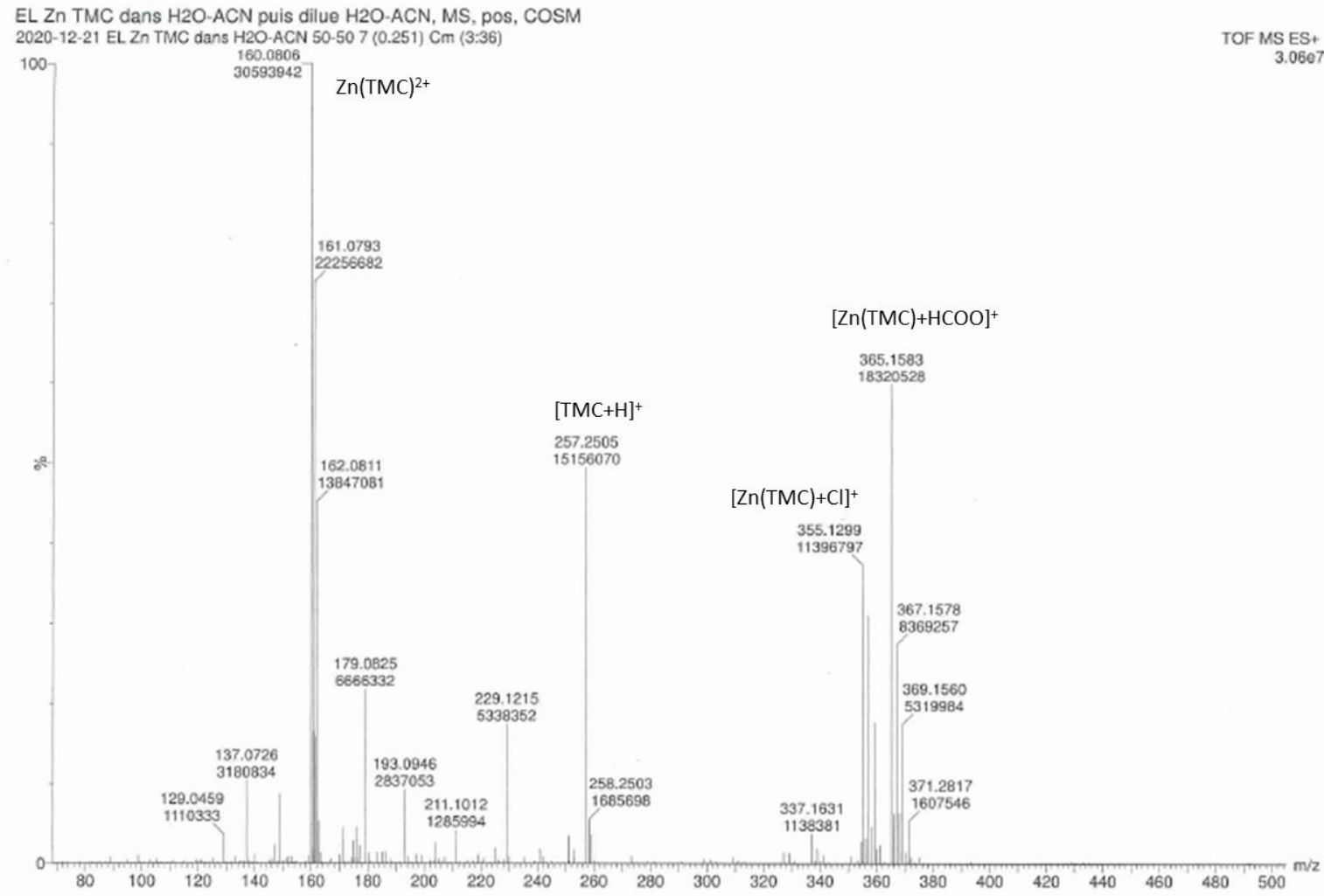

Figure S9. ESI-MS spectrum of $\left[\mathrm{Zn}(\mathbf{T M C})(\mathrm{Cl})_{2}\right]$. 


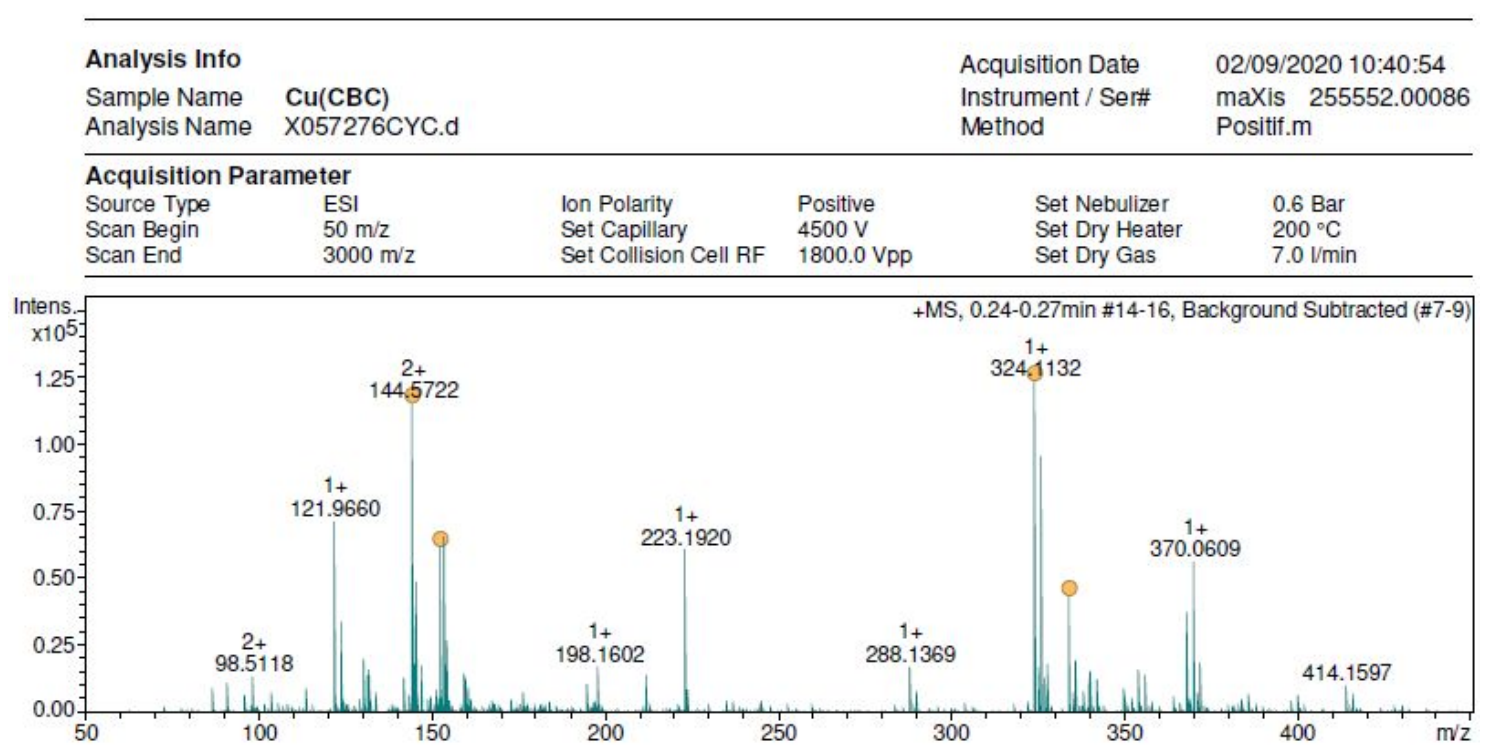

Figure S10. ESI-HRMS spectrum of $\mathrm{Cu}(\mathbf{C B} \text {-cyclam })^{2+}$.

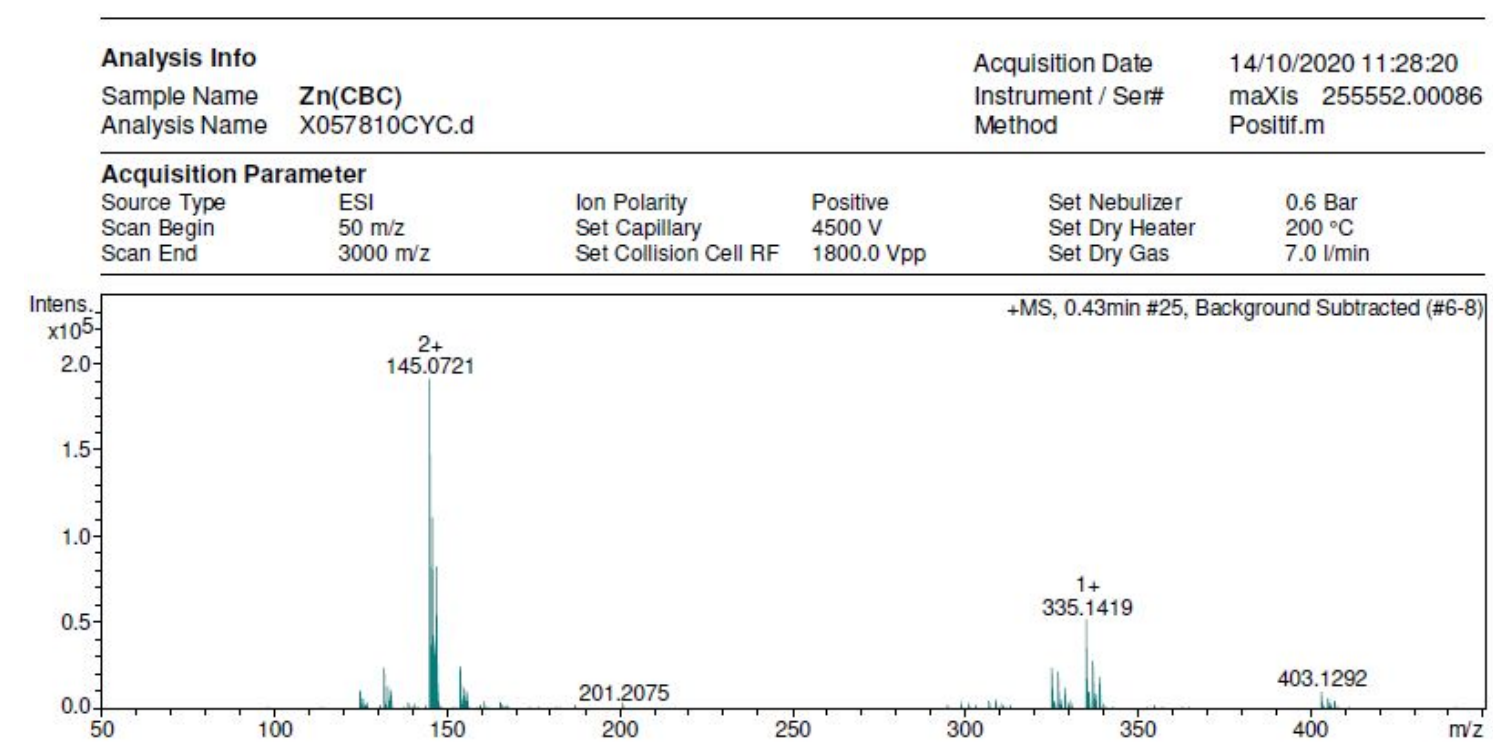

Figure S11. ESI-HRMS spectrum of $\mathrm{Zn}(\mathbf{C B}-\mathbf{c y c l a m})^{2+}$. 


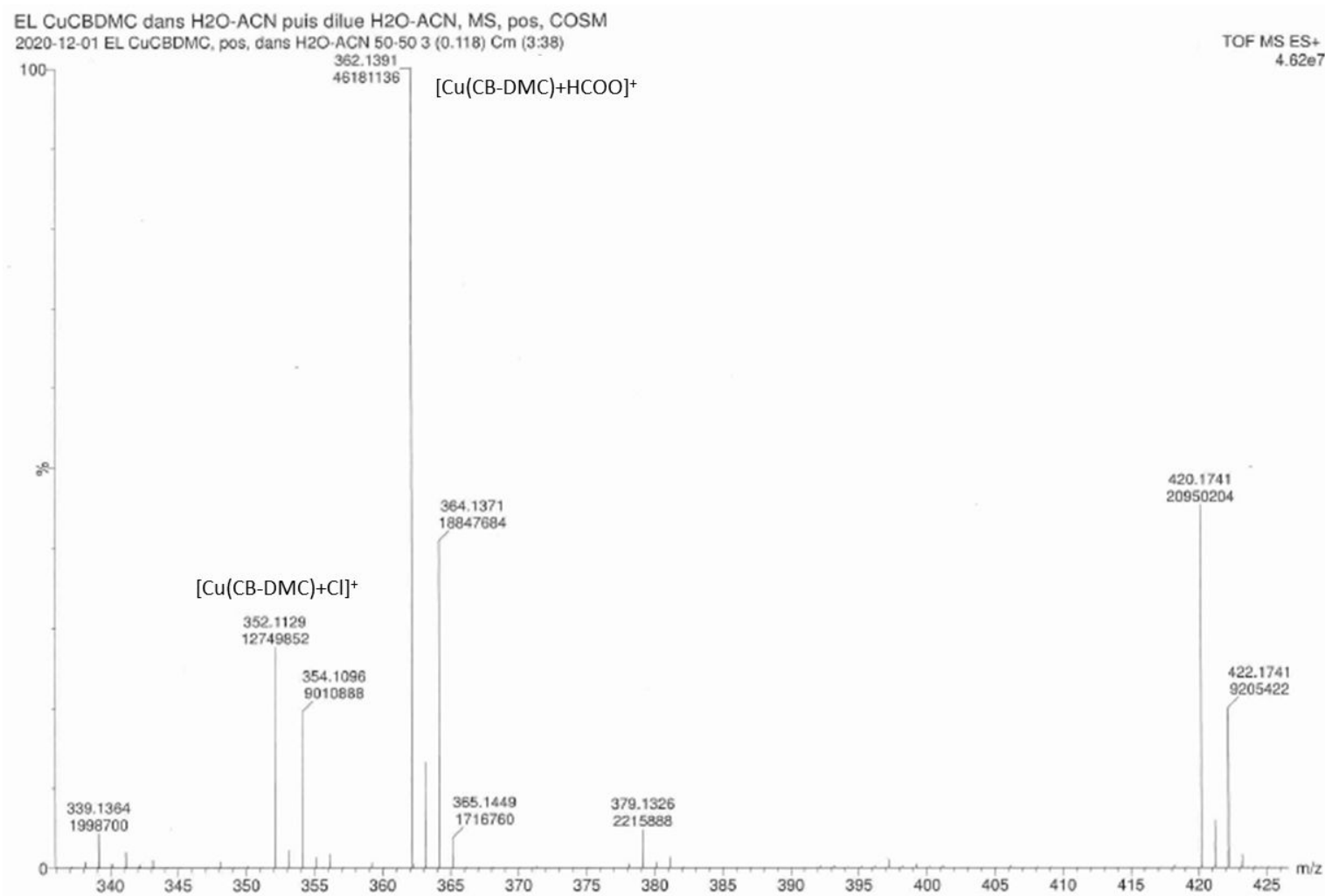

Figure S12. ESI-MS spectrum of $\mathrm{Cu}(\mathbf{C B}-\mathrm{DMC})^{2+}$.

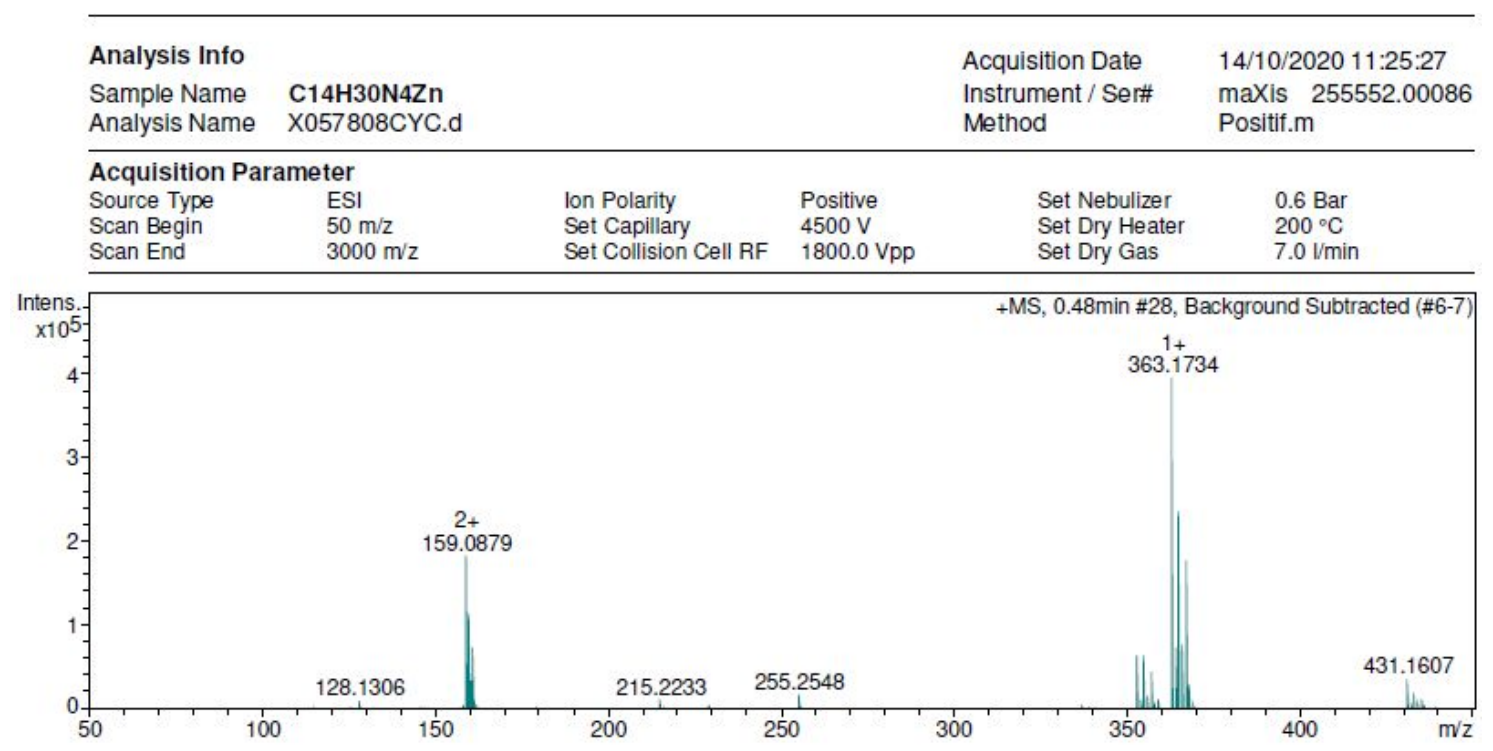

Figure S13. ESI-HRMS spectrum of $\mathrm{Zn}(\mathbf{C B}-\mathrm{DMC})^{2+}$. 


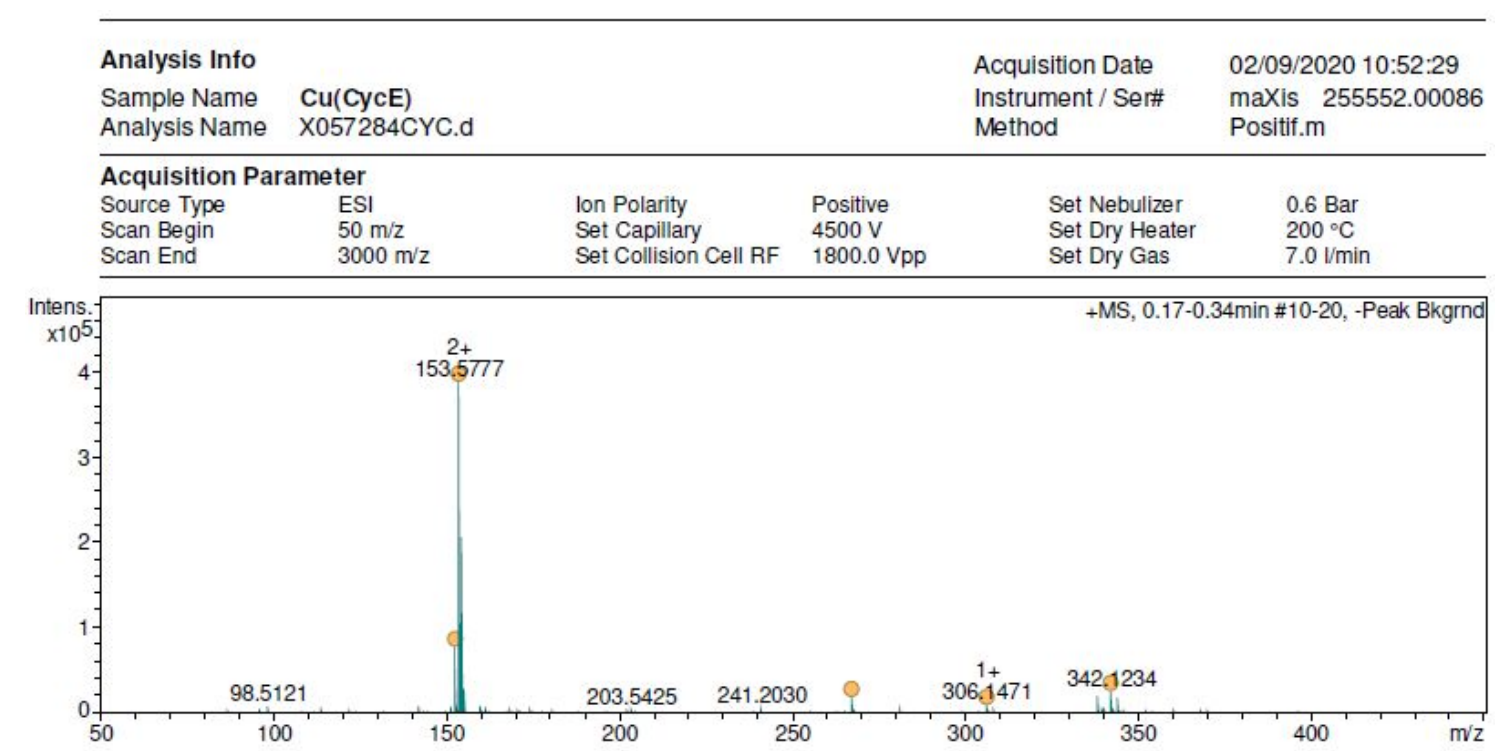

Figure S14. ESI-HRMS spectrum of $\mathrm{Cu}(\text { cyclam-E })^{+}$.

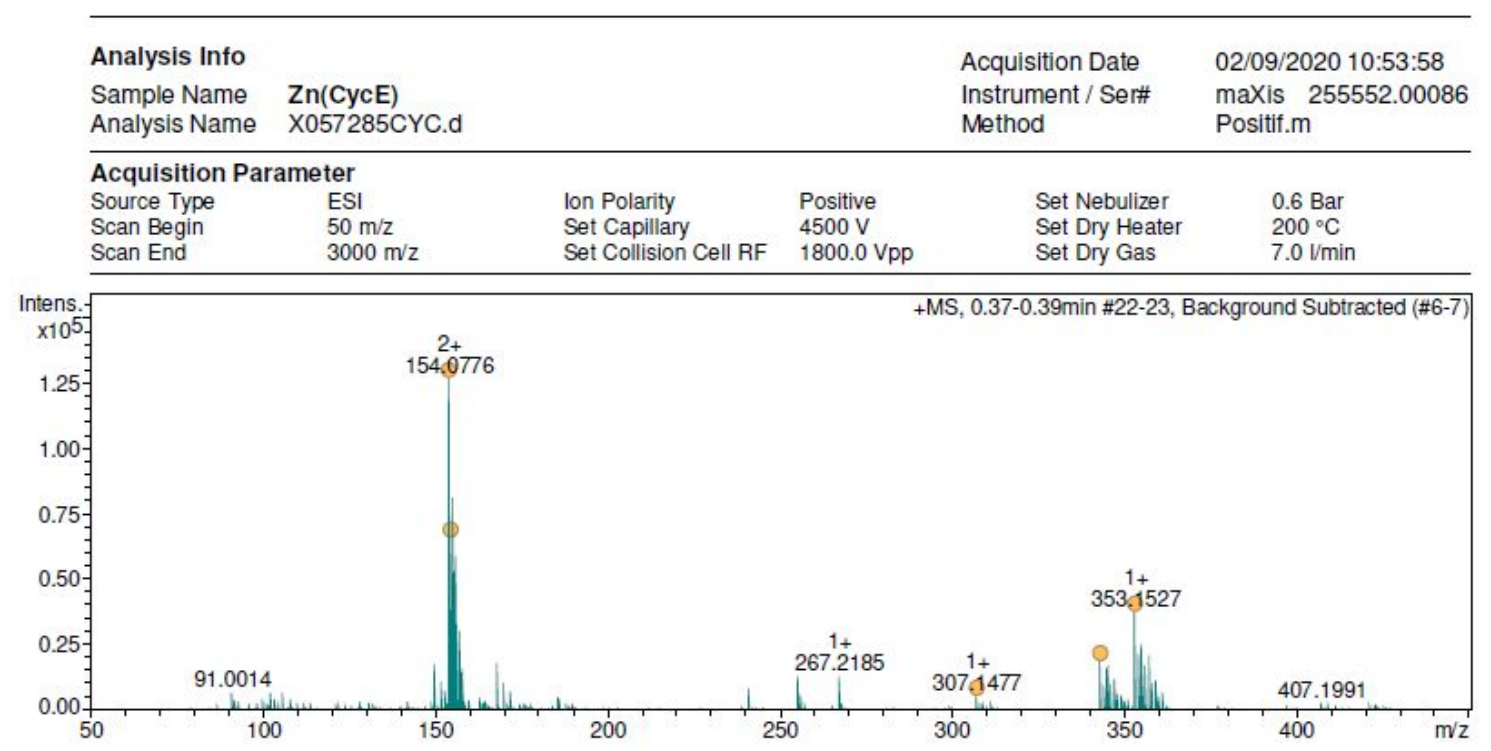

Figure S15. ESI-HRMS spectrum of Zn(cyclam-E $)^{2+}$. 


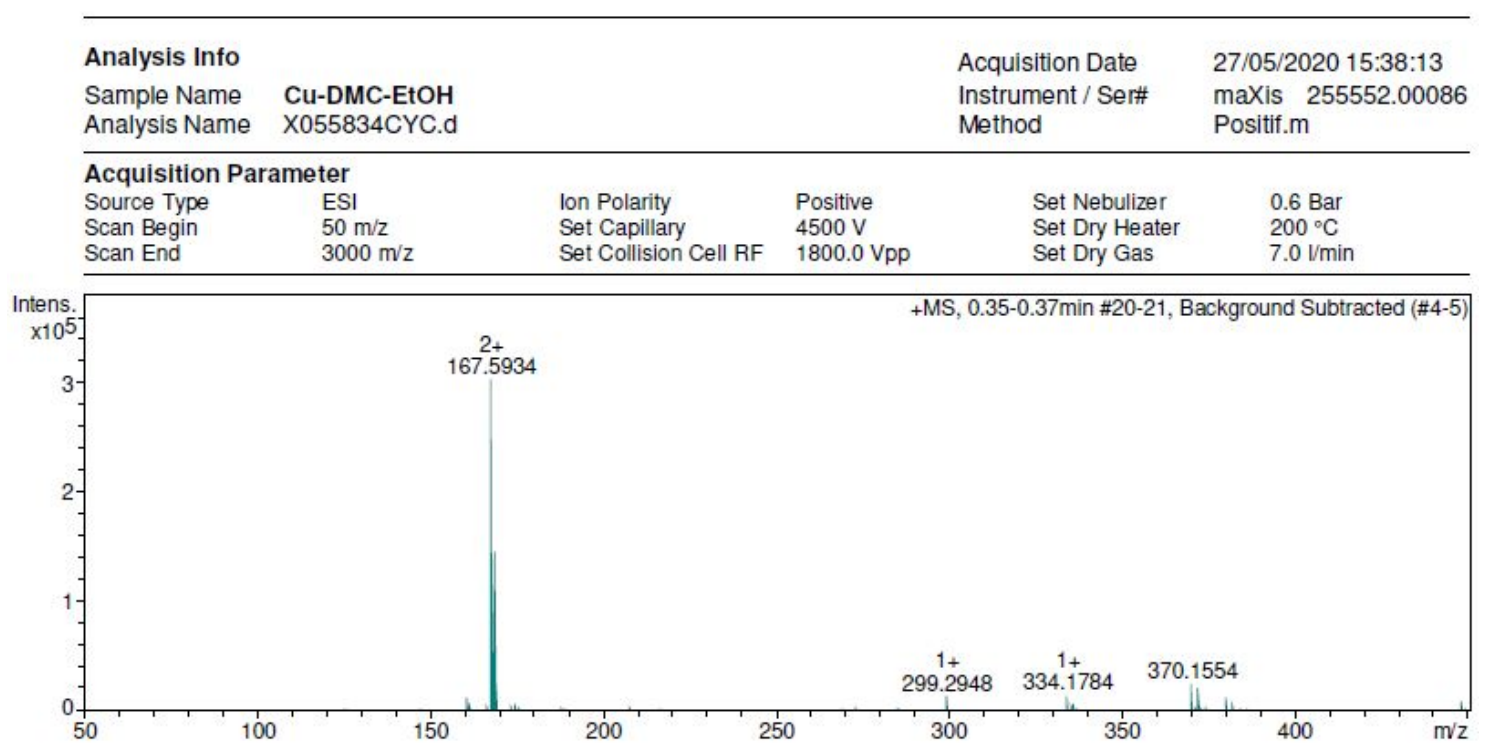

Figure S16. ESI-HRMS spectrum of $\mathrm{Cu}(\text { DMC-E })^{2+}$.

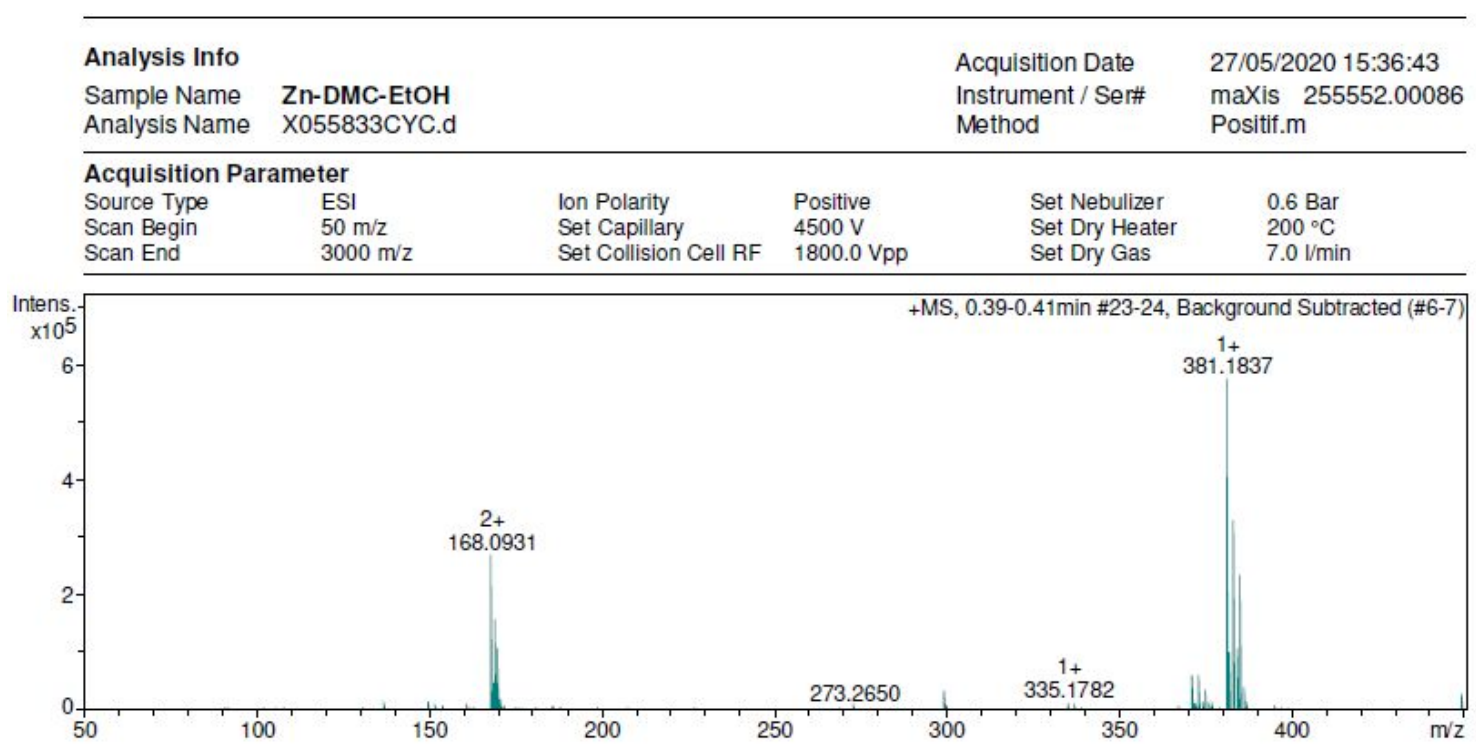

Figure S17. ESI-HRMS spectrum of compound Zn(DMC-E $)^{2+}$. 


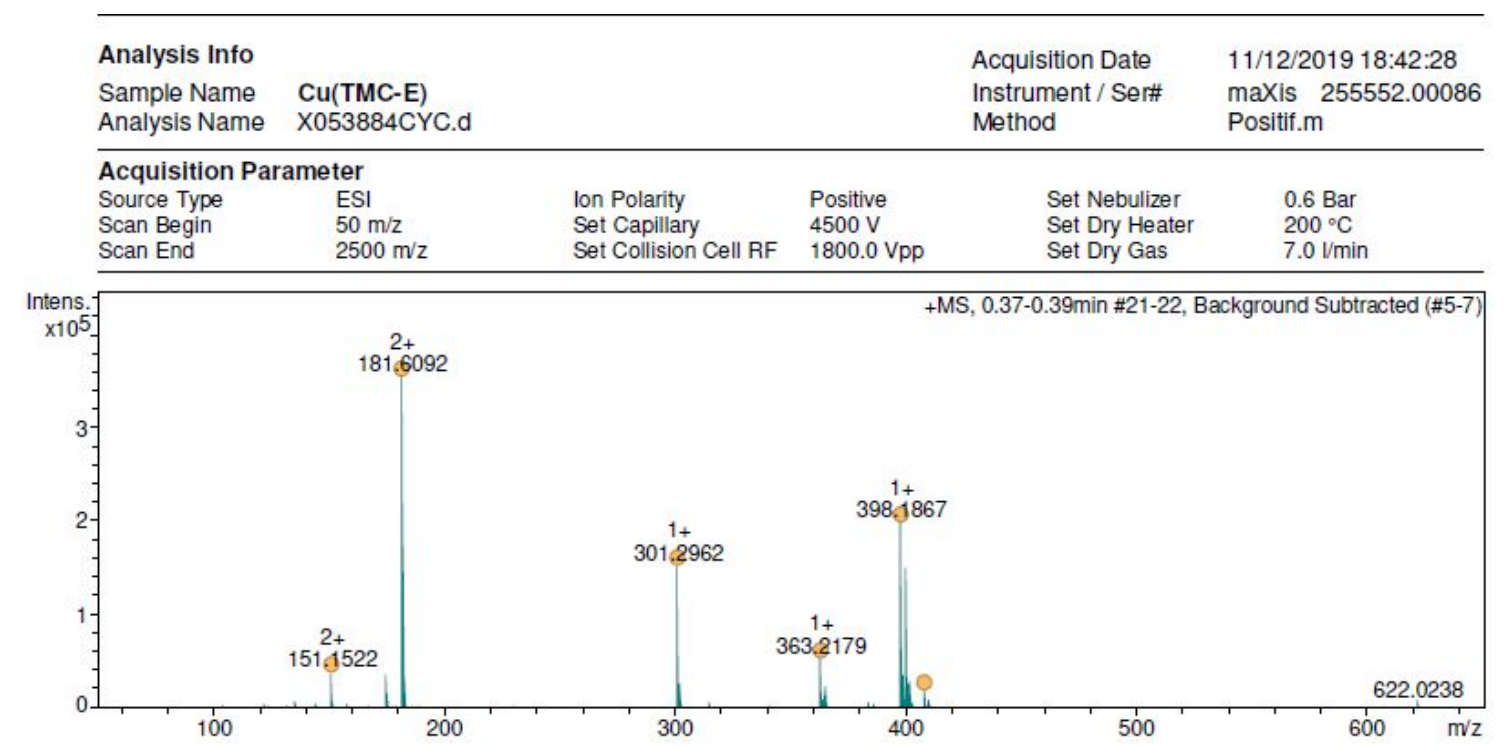

Figure S18. ESI-HRMS spectrum of $\mathrm{Cu}(\mathbf{T M C}-\mathbf{E})^{2+}$.

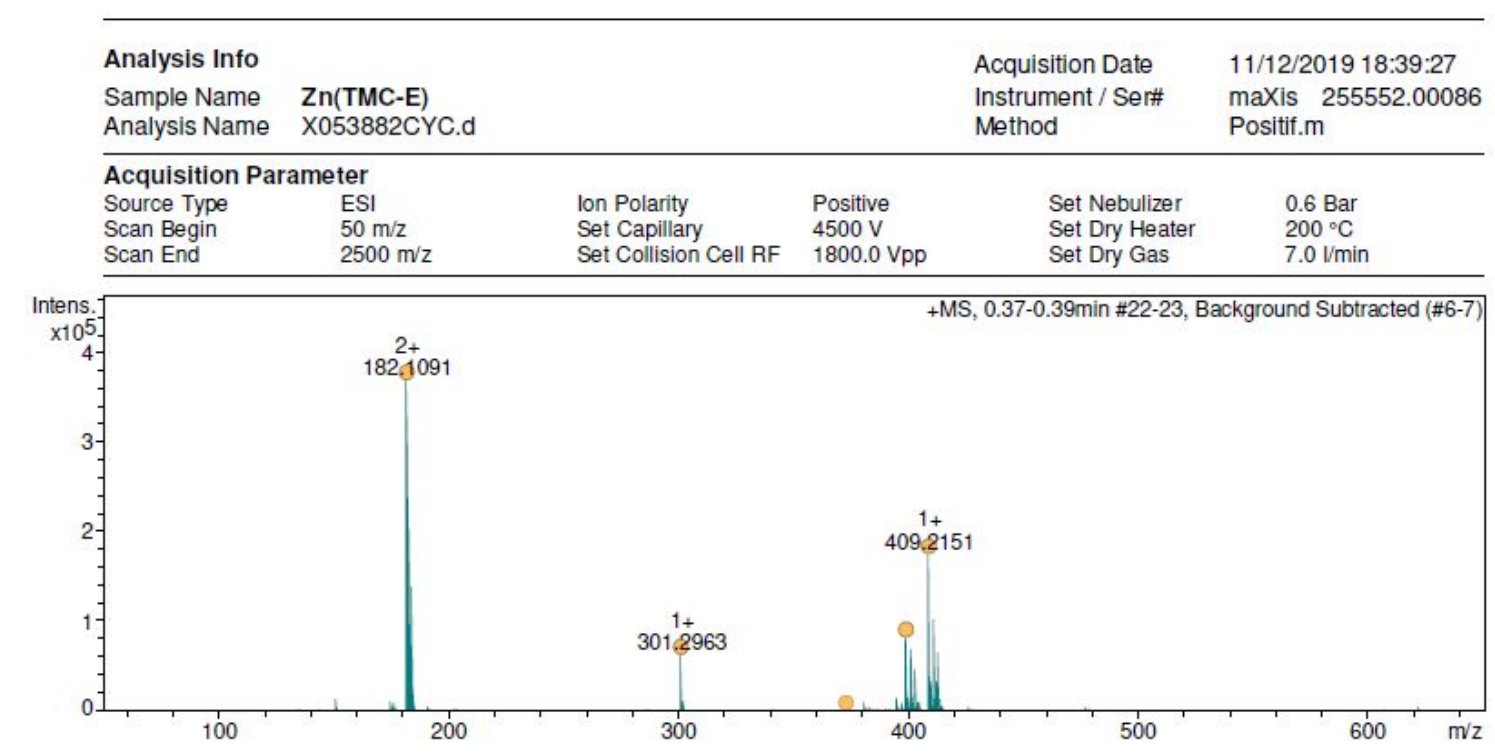

Figure S19. ESI-HRMS spectrum of $\mathrm{Zn}(\text { TMC-E })^{2+}$. 


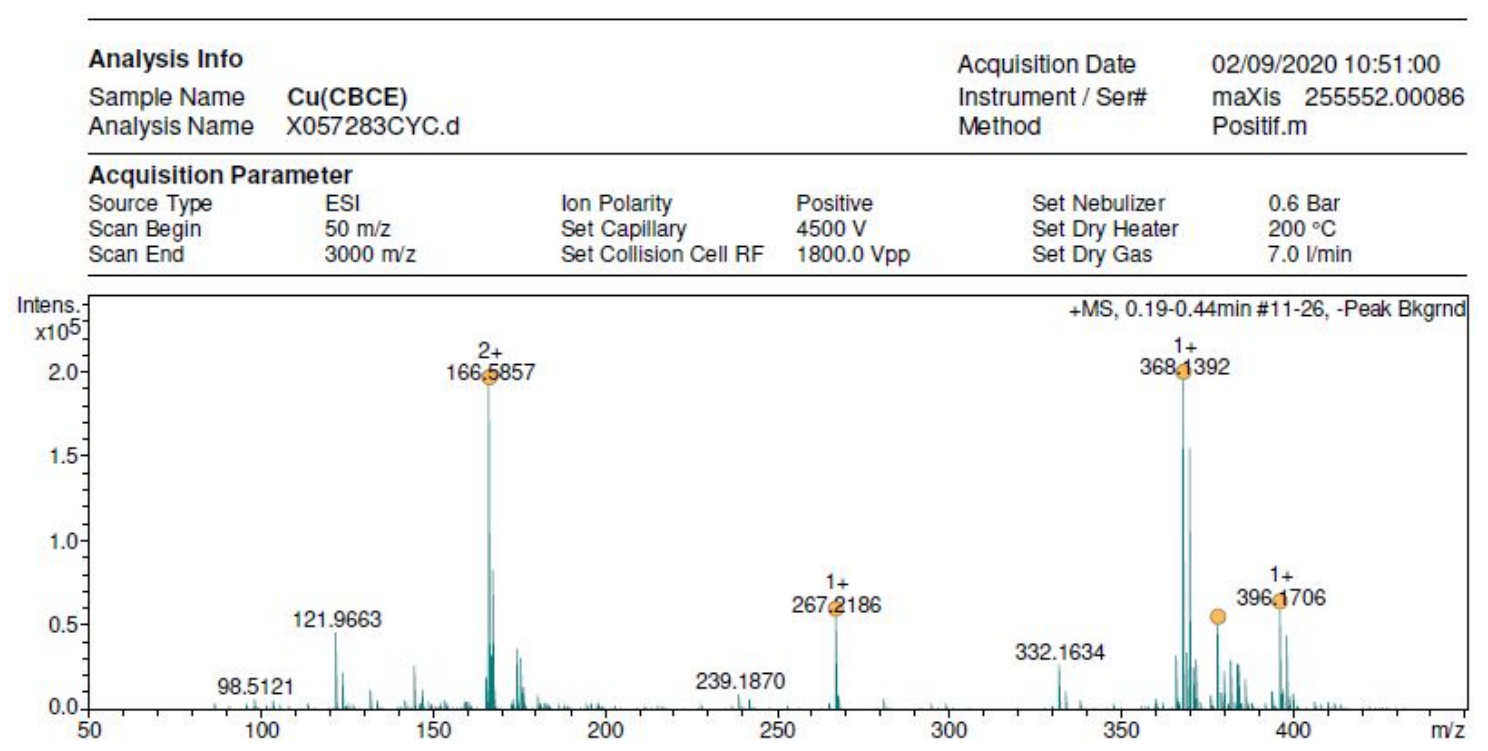

Figure S20. ESI-HRMS spectrum of compound $\mathrm{Cu}(\mathbf{C B}-\text { cyclam-E })^{2+}$.

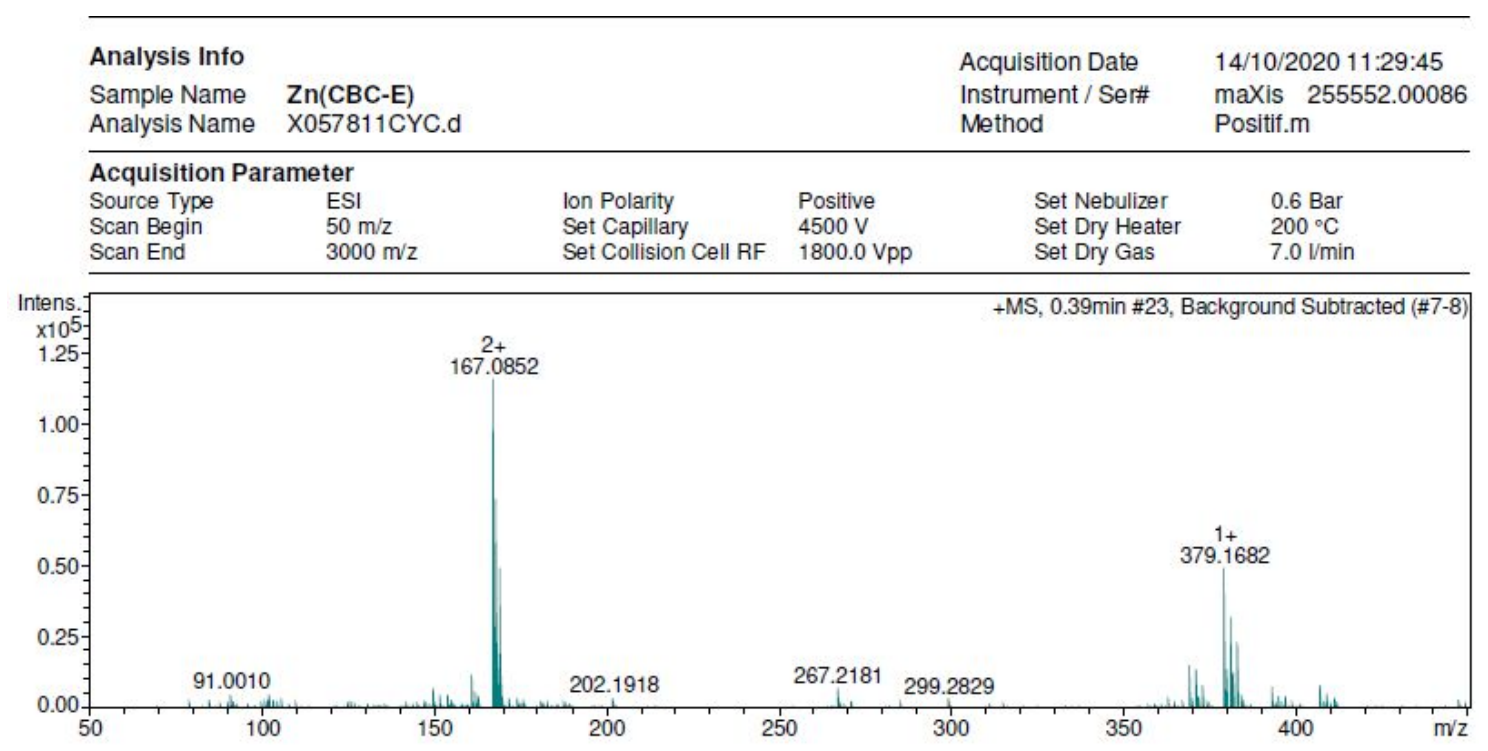

Figure S21. ESI-HRMS spectrum of compound $\mathrm{Zn}(\mathbf{C B}-\mathbf{c y c l a m}-\mathbf{E})^{2+}$. 


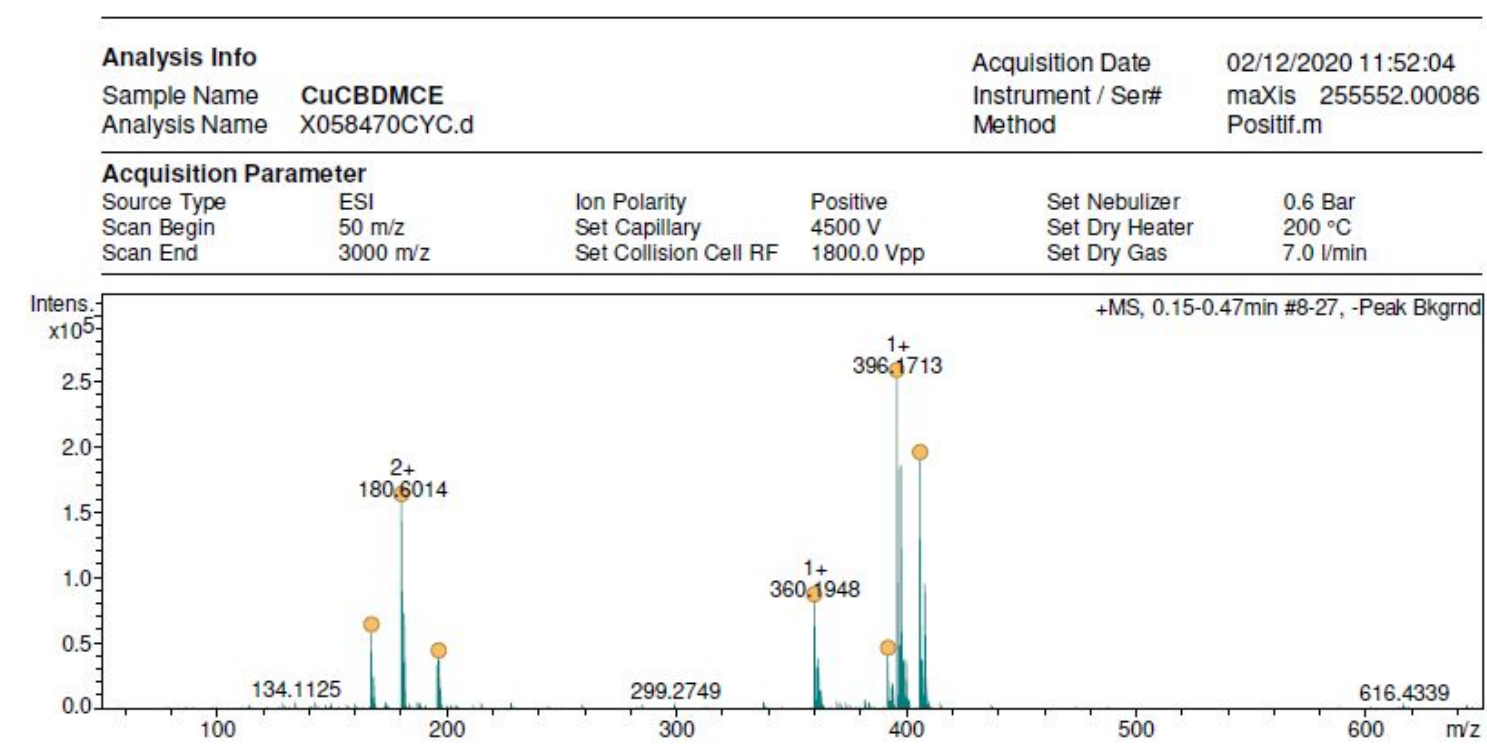

Figure S22. ESI-HRMS spectrum of compound $\mathrm{Cu}(\mathbf{C B}-\mathbf{D M C}-\mathbf{E})^{2+}$.

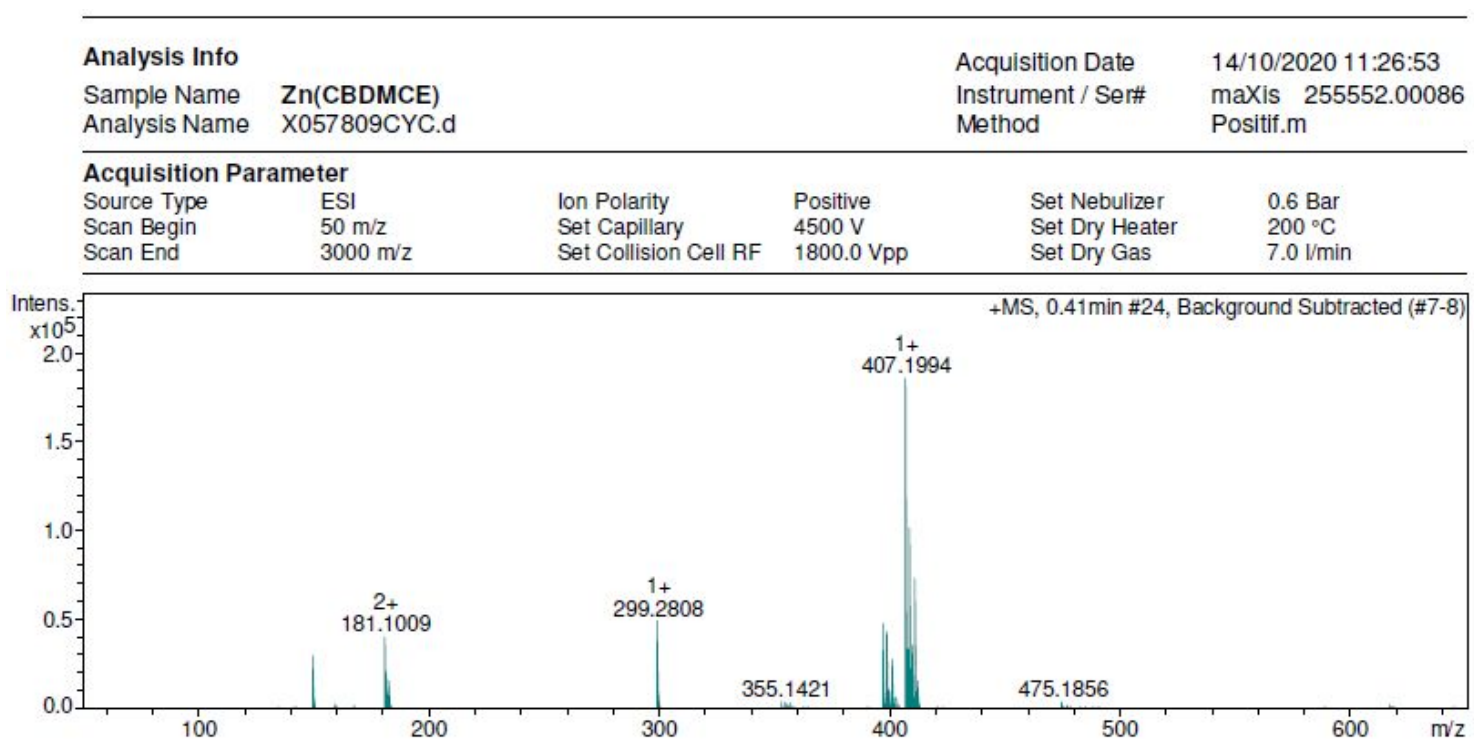

Figure S23. ESI-HRMS spectrum of compound Zn(CB-DMC-E $)^{2+}$. 

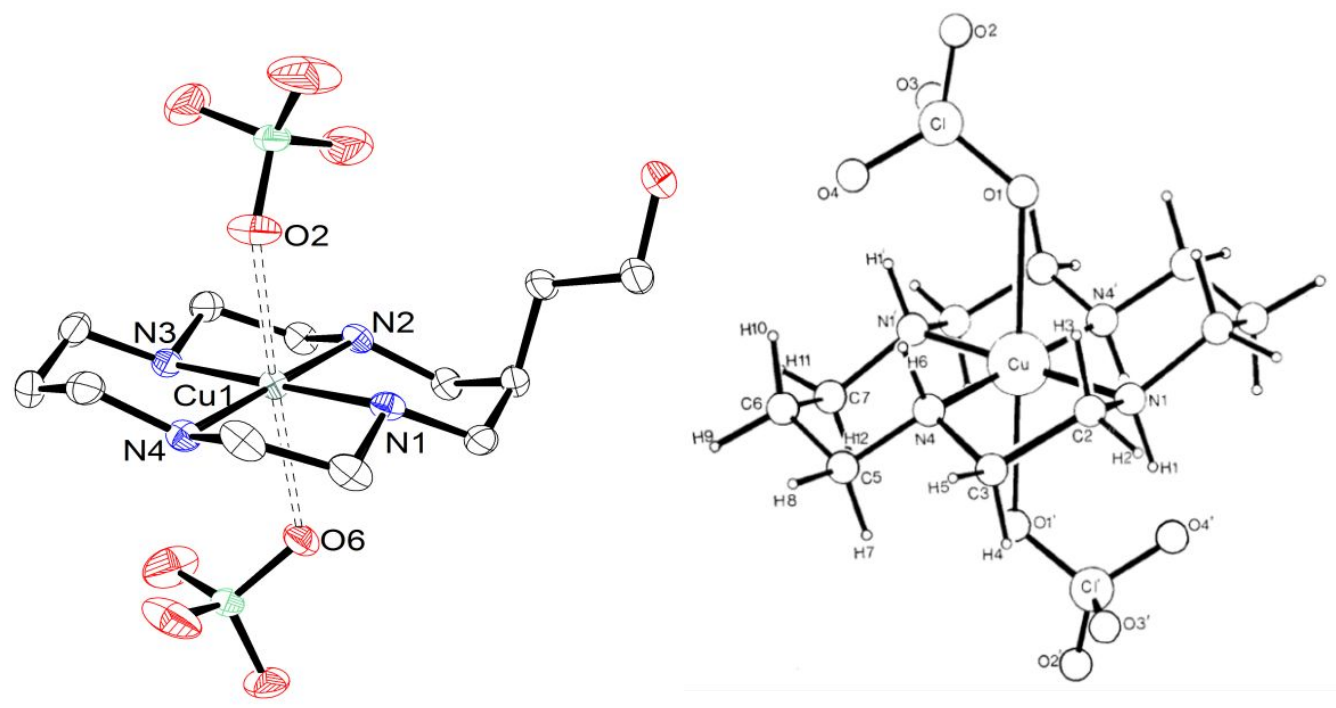

Figure S24. Comparative views of the crystal structures of $\left[\mathrm{Cu}(\mathbf{c y c l a m}-\mathbf{E})\left(\mathrm{ClO}_{4}\right)_{2}\right]$ and $\left[\mathrm{Cu}(\mathbf{c y c l a m})\left(\mathrm{ClO}_{4}\right)_{2}\right]$ from Tripier et al. ${ }^{1}$ and Sklar et al. ${ }^{2}$
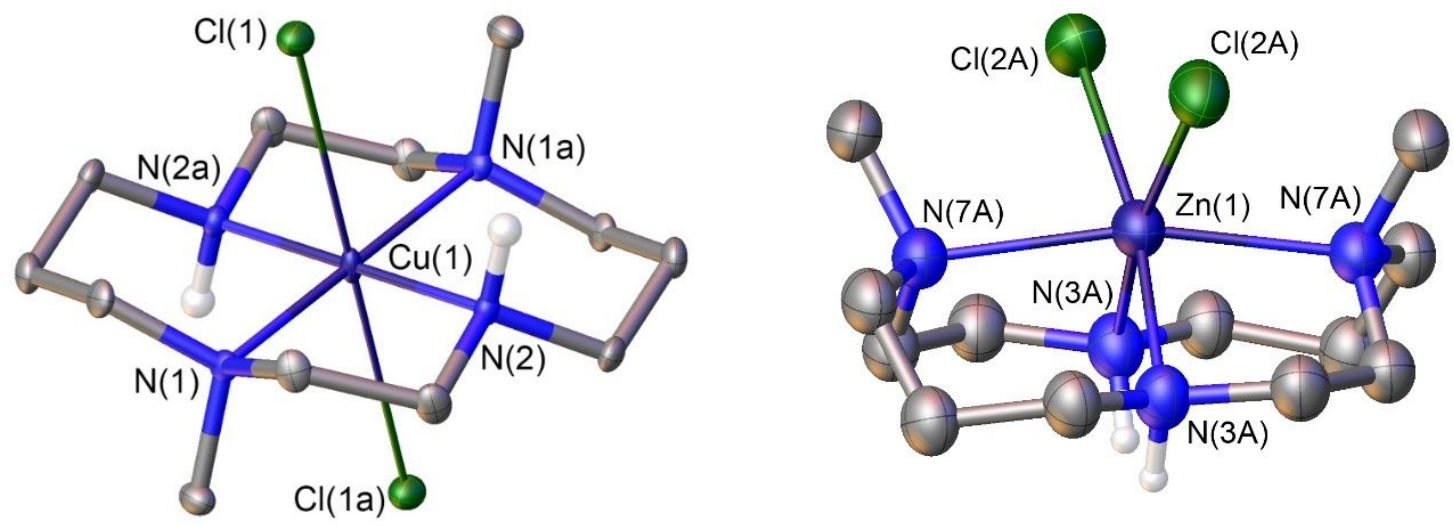

Figure S25. ORTEP views of the crystal structures of $\left.\left[\mathrm{Cu}(\mathbf{D M C})(\mathrm{Cl})_{2}\right)\right]$ (left) and $[\mathrm{Zn}(\mathbf{D M C})$ $\left.(\mathrm{Cl})_{2}\right)$ ] (right; from Lim et al. ${ }^{3}$ )plotted at $50 \%$ ellipsoid probability. Hydrogen atoms bound to $\mathrm{C}$ atoms are omitted for clarity. 
Table S2. Crystallographic data of $\left[(\mathrm{CuDMC}-\mathbf{E})_{2}(\mathrm{Cl})\left(\mathrm{NO}_{3}\right)\right]\left(\mathrm{NO}_{3}\right)_{2}$.

\begin{tabular}{|c|c|}
\hline Empirical formula & $\mathrm{C}_{28} \mathrm{H}_{64} \mathrm{ClCu}_{2} \mathrm{~N}_{11} \mathrm{O}_{11}$ \\
\hline Formula weight & 893.43 \\
\hline Temperature & $130 \mathrm{~K}$ \\
\hline Wavelength & 0.71073 \\
\hline Crystal system & monoclinic \\
\hline \multirow[t]{2}{*}{ Space group } & $\mathrm{I} 2 / \mathrm{a}$ \\
\hline & $\mathrm{a}=16.4888(9) \AA$ \\
\hline \multirow[t]{2}{*}{ Unit cell dimensions } & $\mathrm{b}=14.1520(7) \AA$ \\
\hline & $\mathrm{c}=17.5606(9) \AA$ \\
\hline Volume & $4026.6(4) \AA^{3}$ \\
\hline Z & 4 \\
\hline Density (calculated) & $1.474 \mathrm{mg} / \mathrm{m}^{3}$ \\
\hline Absorption coefficient & $1.189 \mathrm{~mm}^{-1}$ \\
\hline $\mathrm{F}(000)$ & 1888 \\
\hline Crystal size & $0.40 \times 0.19 \times 0.11 \mathrm{~mm}$ \\
\hline$\theta$ range for data collection & 3.27 to $26.37^{\circ}$ \\
\hline Index ranges & $-20<=\mathrm{h}<=20,-17<=\mathrm{k}<=17,-21<=\mathrm{l}<=21$ \\
\hline Reflections collected & 14359 \\
\hline Independent reflections & $4112[\mathrm{R}(\mathrm{int})=0.0564]$ \\
\hline Completeness to $\theta=25.242^{\circ}$ & 99.8 \\
\hline Absorption correction & MULTI-SCAN \\
\hline Max. and min. transmission & 0.880 and 0.439 \\
\hline Refinement method & Full-matrix least-squares on $\mathrm{F}^{2}$ \\
\hline Data/restraints/parameters & $4112 / 571 / 242$ \\
\hline Goodness-of-fit on $\mathrm{F}^{2}$ & 1.349 \\
\hline Final $R$ indices $[\mathrm{I}>2 \operatorname{sigma}(\mathrm{I})]$ & $\mathrm{R} 1=0.127, \mathrm{wR} 2=0.3623$ \\
\hline $\mathrm{R}$ indices (all data) & $\mathrm{R} 1=0.17, \mathrm{wR} 2=0.4089$ \\
\hline \multicolumn{2}{|l|}{ Extinction coefficient } \\
\hline Largest diff. peak and hole & 0.825 and $-0.469 \mathrm{e} \cdot \AA^{-3}$ \\
\hline
\end{tabular}


Table S3. Crystallographic data of $[\mathrm{Cu}(\mathbf{T M C}-\mathbf{E}) \mathrm{Cl}]\left(\mathrm{NO}_{3}\right)$.

\begin{tabular}{|c|c|}
\hline Empirical formula & $\mathrm{C}_{16} \mathrm{H}_{36} \mathrm{ClCuI}_{0.34} \mathrm{~N}_{4.66} \mathrm{O}_{2.98}$ \\
\hline Formula weight & 483.56 \\
\hline Temperature & $133(2) \mathrm{K}$ \\
\hline Wavelength & $0.71073 \AA$ \\
\hline Crystal system & Orthorhombic \\
\hline \multirow[t]{2}{*}{ Space group } & P n m a \\
\hline & $\mathrm{a}=22.5502(10) \AA$ \\
\hline \multirow[t]{2}{*}{ Unit cell dimensions } & $\mathrm{b}=10.7372(5) \AA$ \\
\hline & $\mathrm{c}=8.6348(4) \AA$ \\
\hline Volume & $2090.71(17) \AA^{3}$ \\
\hline $\mathrm{Z}$ & 4 \\
\hline Density (calculated) & $1.505 \mathrm{mg} / \mathrm{m}^{3}$ \\
\hline Absorption coefficient & $1.699 \mathrm{~mm}^{-1}$ \\
\hline $\mathrm{F}(000)$ & 994 \\
\hline Crystal size & $0.14 \times 0.11 \times 0.07 \mathrm{~mm}$ \\
\hline$\theta$ range for data collection & 3.53 to $26.36^{\circ}$ \\
\hline Index ranges & $\begin{array}{l}-28<=\mathrm{h}<=24,-13<=\mathrm{k}<=11,- \\
10<=\mathrm{l}<=10\end{array}$ \\
\hline Reflections collected & 13485 \\
\hline Independent reflections & $2250[\mathrm{R}(\mathrm{int})=0.0553]$ \\
\hline Completeness to $\theta=25.242^{\circ}$ & $99.7 \%$ \\
\hline Absorption correction & Analytical \\
\hline Max. and min. transmission & 0.69 and 0.505 \\
\hline Refinement method & Full-matrix least-squares on $\mathrm{F}^{2}$ \\
\hline Data/restraints/parameters & $2250 / 133 / 225$ \\
\hline Goodness-of-fit on $\mathrm{F}^{2}$ & 1.096 \\
\hline Final $R$ indices $[\mathrm{I}>2 \operatorname{sigma}(\mathrm{I})]$ & $\mathrm{R} 1=0.0626, \mathrm{wR} 2=0.1584$ \\
\hline $\mathrm{R}$ indices (all data) & $\mathrm{R} 1=0.0796, \mathrm{wR} 2=0.1695$ \\
\hline Extinction coefficient & n.a. \\
\hline Largest diff. peak and hole & 1.176 and $-0.603 \mathrm{e} \cdot \AA^{-3}$ \\
\hline
\end{tabular}


Table S4. Crystallographic data of $[\mathrm{Zn}(\mathbf{T M C}-\mathbf{E}) \mathrm{Cl}]\left(\mathrm{NO}_{3}\right)$.

\begin{tabular}{|c|c|}
\hline Empirical formula & $\mathrm{C}_{64} \mathrm{H}_{144} \mathrm{Cl}_{5} \mathrm{~N}_{19} \mathrm{O}_{13} \mathrm{Zn}_{4}$ \\
\hline Formula weight & 1826.71 \\
\hline Temperature & $150(2) \mathrm{K}$ \\
\hline Wavelength & $0.71073 \AA$ \\
\hline Crystal system & Monoclinic \\
\hline \multirow[t]{2}{*}{ Space group } & $\mathrm{P} 21 / \mathrm{n}$ \\
\hline & $\mathrm{a}=8.8664(4) \AA$ \\
\hline \multirow[t]{2}{*}{ Unit cell dimensions } & $\mathrm{b}=22.2898(9) \AA$ \\
\hline & $c=10.7157(5) \AA$ \\
\hline Volume & $2117.39(16) \AA^{3}$ \\
\hline $\mathrm{Z}$ & 1 \\
\hline Density (calculated) & $1.433 \mathrm{mg} / \mathrm{m}^{3}$ \\
\hline Absorption coefficient & $1.344 \mathrm{~mm}^{-1}$ \\
\hline $\mathrm{F}(000)$ & 970 \\
\hline Crystal size & $0.29 \times 0.16 \times 0.15 \mathrm{~mm}^{3}$ \\
\hline$\theta$ range for data collection & 3.34 to $26.37^{\circ}$ \\
\hline Index ranges & $\begin{array}{l}-9<=\mathrm{h}<=11,-27<=\mathrm{k}<=27,- \\
13<=1<=13\end{array}$ \\
\hline Reflections collected & 21603 \\
\hline Independent reflections & $4320[\mathrm{R}($ int $)=0.0890]$ \\
\hline Completeness to $\theta=25.242^{\circ}$ & $99.8 \%$ \\
\hline Absorption correction & Analytical \\
\hline Max. and min. transmission & 0.8239 and 0.6966 \\
\hline Refinement method & Full-matrix least-squares on $\mathrm{F}^{2}$ \\
\hline Data/restraints/parameters & 4320 / 0 / 249 \\
\hline Goodness-of-fit on $\mathrm{F}^{2}$ & 1.143 \\
\hline Final $\mathrm{R}$ indices [I>2sigma $(\mathrm{I})]$ & $\mathrm{R} 1=0.0469, \mathrm{wR} 2=0.1314$ \\
\hline $\mathrm{R}$ indices (all data) & $\mathrm{R} 1=0.0534, \mathrm{wR} 2=0.1369$ \\
\hline Extinction coefficient & n.a. \\
\hline Largest diff. peak and hole & 1.009 and $-0.412 \mathrm{e} \cdot \AA^{-3}$ \\
\hline
\end{tabular}



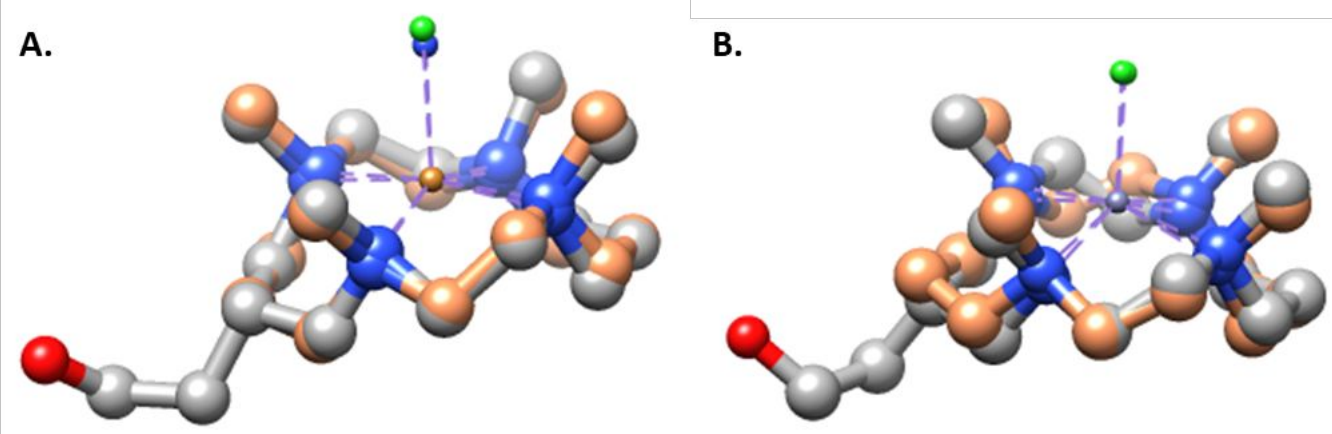

Figure S26. Superposition of the X-ray crystal structures of (A) $[\mathrm{Cu}(\mathrm{TMC})(\mathrm{NCS})]^{+}$(beige) and $[\mathrm{Cu}(\mathbf{T M C}-\mathbf{E}) \mathrm{Cl}]^{+}$(gray) and (B) $[\mathrm{Zn}(\mathbf{T M C}) \mathrm{Cl}]^{+}$(beige) and $[\mathrm{Zn}(\mathbf{T M C}-\mathbf{E}) \mathrm{Cl}]^{+}$(gray). The superposition is based on the best superposition of the 4 nitrogen atoms. Water molecules, noncoordinating counter ions, and hydrogen atoms have been omitted for clarity. The TMC complexes are adapted from $\mathrm{Lu}$ et $\mathrm{al} .{ }^{4}$ and Moore et al. ${ }^{5}$ 
Table S5. Crystallographic data of $\left[\mathrm{Zn}(\mathbf{C B}-\text { cyclam-E })_{2}(\mathrm{Cl})_{2}\right] \mathrm{Cl}_{2}$.

\begin{tabular}{ll}
\hline Empirical formula & $\mathrm{C}_{32} \mathrm{H}_{66} \mathrm{Cl}_{4} \mathrm{~N}_{8} \mathrm{O}_{2} \mathrm{Zn}_{2}$ \\
Formula weight & 895.48 \\
Temperature & $150(2) \mathrm{K}$ \\
Wavelength & $0.71073 \AA$ \\
Crystal system & Monoclinic \\
Space group & $\mathrm{P} 21 / \mathrm{c}$ \\
& $\mathrm{a}=9.1627(8) \AA$ \\
Unit cell dimensions & $\mathrm{b}=20.5046(18) \AA \quad \beta=106.902(4))^{\circ}$ \\
& $\mathrm{c}=11.4454(10) \AA$ \\
Volume & $2057.4(3) \AA^{3}$ \\
Z & 2 \\
Density (calculated) & $1.445 \mathrm{~g} / \mathrm{cm}^{3}$ \\
Absorption coefficient & $1.468 \mathrm{~mm}{ }^{-1}$ \\
F(000) & 944 \\
Crystal size & $0.350 \mathrm{x} 0.240 \mathrm{x} 0.170 \mathrm{~mm}$ \\
$\theta$ range for data collection & 2.527 to $27.491{ }^{\circ}$ \\
Index ranges & $-11<=\mathrm{h}<=11,-26<=\mathrm{k}<=26,-$ \\
Reflections collected & $14<=1<=14$ \\
Independent reflections & 14541 \\
Completeness to $\theta=25.242^{\circ}$ & $4683[\mathrm{R}(\mathrm{int})=0.0313]$ \\
Absorption correction & 0.993 \\
Max. and min. transmission & $0.779,0.705$ \\
Refinement method & $\mathrm{Full-matrix} \mathrm{least-squares} \mathrm{on} \mathrm{F}^{\wedge} 2$ \\
Data/restraints/parameters & $4683 / 41 / 275$ \\
Goodness-of-fit on $\mathrm{F}^{2}$ & 1.034 \\
Final R indices [I $>2 \mathrm{sigma}(\mathrm{I})]$ & $\mathrm{R} 1=0.0380, \mathrm{wR} 2=0.0876$ \\
R indices (all data) & $\mathrm{R} 1=0.0503, \mathrm{wR} 2=0.0929$ \\
Extinction coefficient & $\mathrm{n} . \mathrm{a}$. \\
Largest diff. peak and hole & $0.660 \mathrm{and}-0.449 \mathrm{e} . \AA^{-3}$ \\
\hline
\end{tabular}


A.

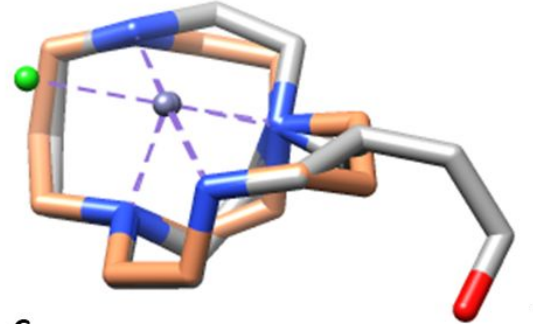

c.

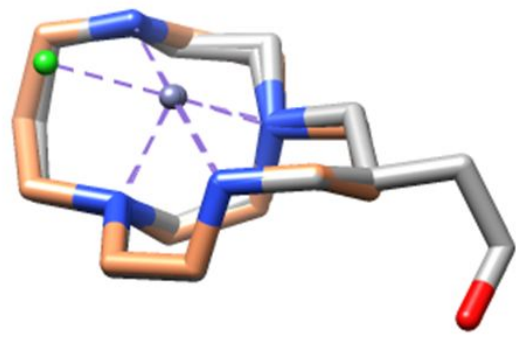

B.

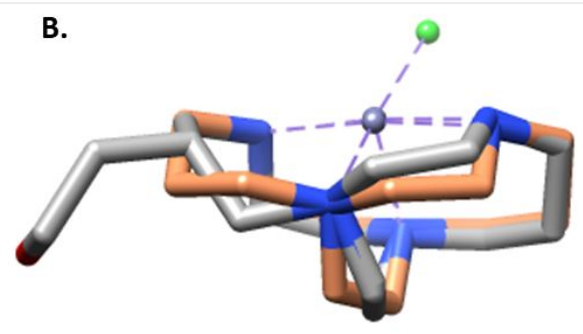

D.

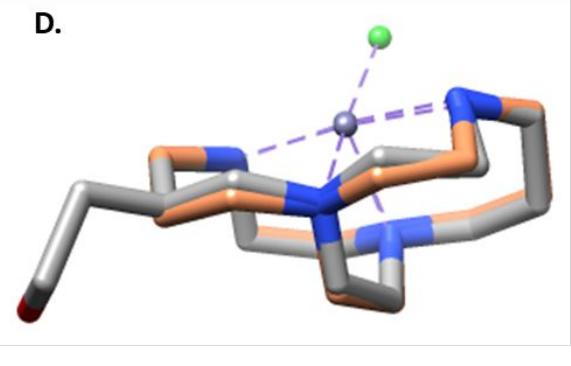

Figure 27. Superposition of the structures of $[\mathrm{Zn}(\mathbf{C B}-\mathbf{c y c l a m}) \mathrm{Cl}]^{+}$with: (A) / (B) the cis-isomer of $[\mathrm{Zn}(\mathbf{C B}-\mathbf{c y c l a m}-\mathrm{E}) \mathrm{Cl}]^{+}$and $(\mathrm{C}) /(\mathrm{D})$ the anti-isomer of $[\mathrm{Zn}(\mathbf{C B}-\mathbf{c y c l a m}-\mathbf{E}) \mathrm{Cl}]^{+}$. Structure of $[\mathrm{Zn}(\mathbf{C B} \text {-cyclam }) \mathrm{Cl}]^{+}$is adapted from Rheingold et al. ${ }^{6}$ 

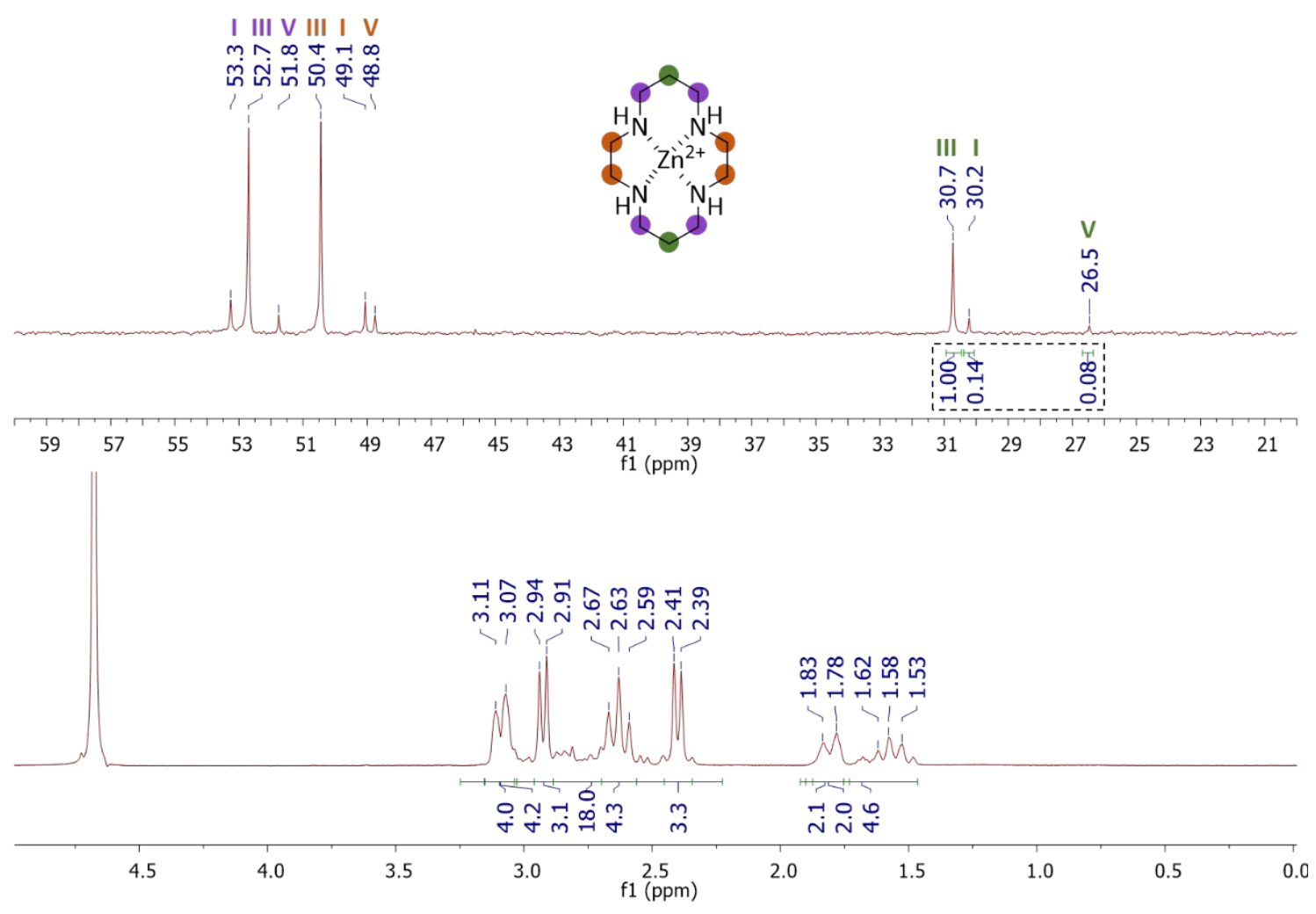

Figure S28. $\mathrm{NMR}{ }^{13} \mathrm{C}$ Jmod and ${ }^{1} \mathrm{H}\left(75\right.$ and $\left.300 \mathrm{MHz}, \mathrm{D}_{2} \mathrm{O}, 298 \mathrm{~K}\right)$ spectra of $\left[\mathrm{Zn}(\mathbf{c y c l a m}) \mathrm{Cl}_{2}\right]$. The identification of the three conformations present in solution and the attribution of the chemical shifts have been done following Sadler et al. ${ }^{7}$ 


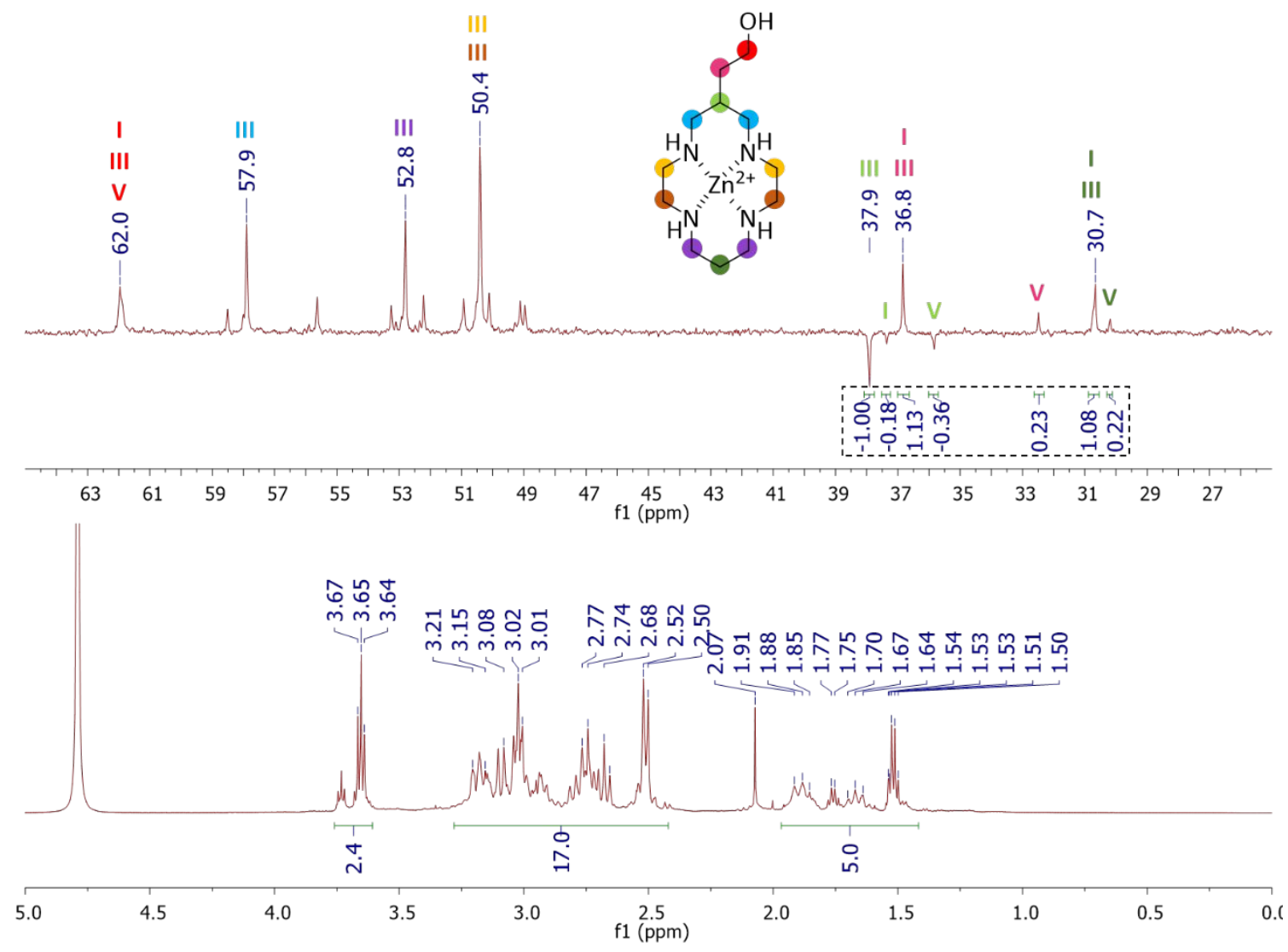

Figure S29. NMR ${ }^{13} \mathrm{C}$ Jmod and ${ }^{1} \mathrm{H}\left(125\right.$ and $\left.500 \mathrm{MHz}, \mathrm{D}_{2} \mathrm{O}, 298 \mathrm{~K}\right)$ spectra of $\left[\mathrm{Zn}(\mathbf{c y c l a m}-\mathbf{E}) \mathrm{Cl}_{2}\right]$. The attribution of the chemical shifts was carried out thanks to the $2 \mathrm{D}$ NMR analyzes and the identification of the conformations by deduction from the data of [Zn(cyclam $) \mathrm{Cl}_{2}$ ]. 

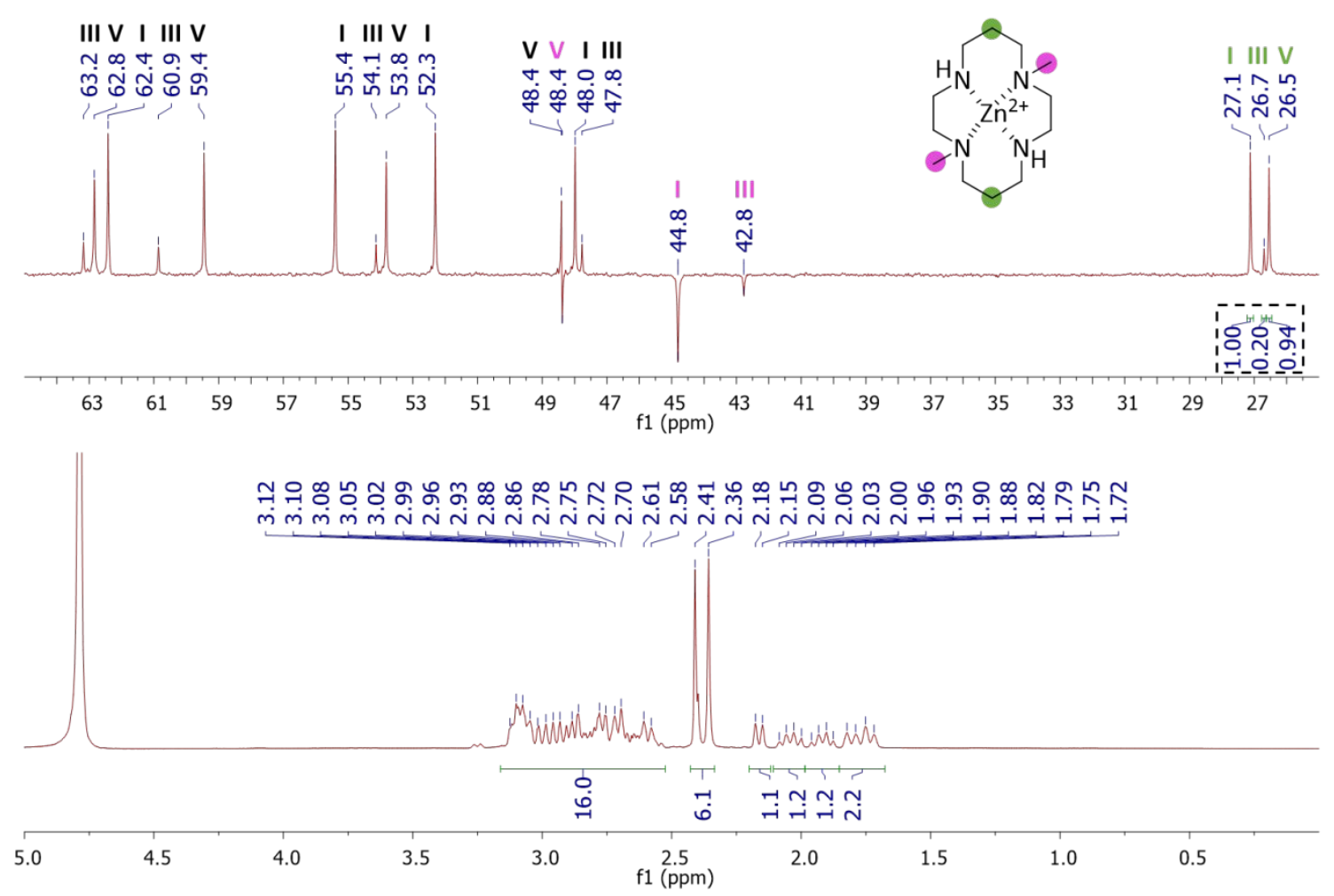

Figure S30. NMR ${ }^{13} \mathrm{C}$ Jmod and ${ }^{1} \mathrm{H}\left(125\right.$ and $\left.500 \mathrm{MHz}, \mathrm{D}_{2} \mathrm{O}, 298 \mathrm{~K}\right)$ spectra of $\left[\mathrm{Zn}(\mathbf{D M C}) \mathrm{Cl}_{2}\right]$. The identification of the isomers was made by deduction from the previous analyzes. 

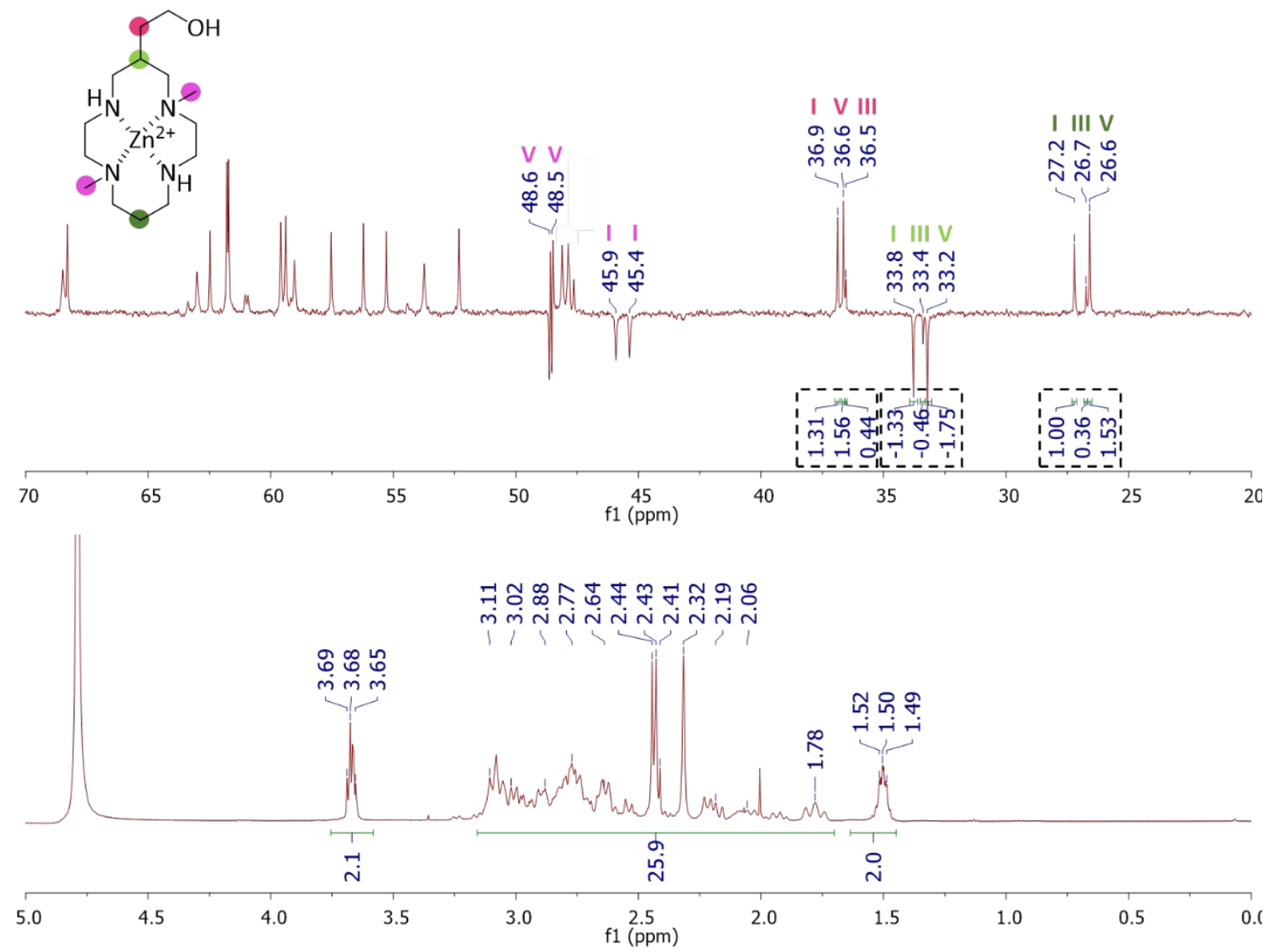

Figure S31. NMR ${ }^{13} \mathrm{C}$ Jmod and ${ }^{1} \mathrm{H}\left(125\right.$ and $\left.500 \mathrm{MHz}, \mathrm{D}_{2} \mathrm{O}, 298 \mathrm{~K}\right)$ spectra of [Zn(DMCE) $\mathrm{Cl}_{2}$ ]. The identification of the isomers was made by deduction from the previous analyzes. 

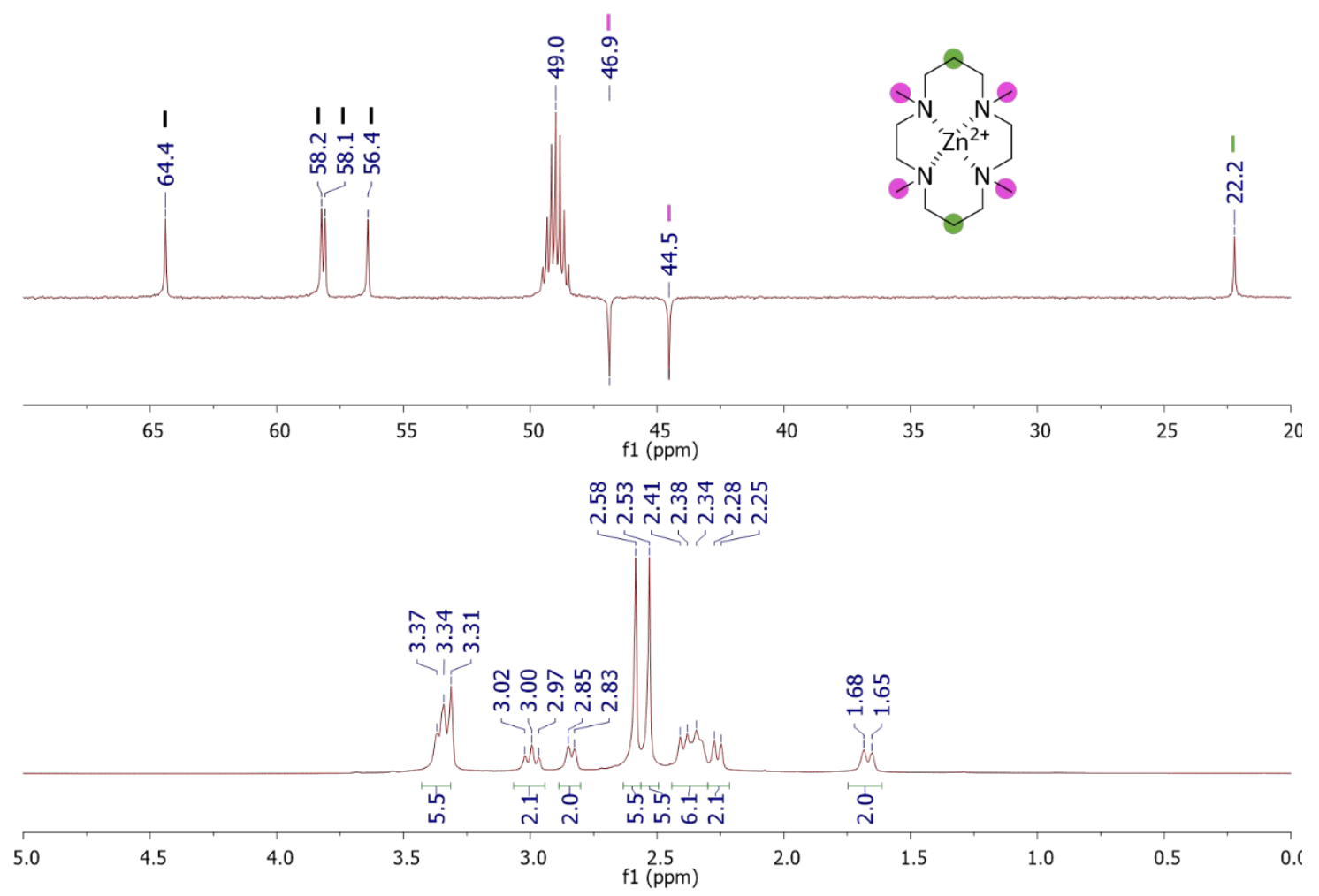

Figure S32. NMR ${ }^{13} \mathrm{C}$ Jmod and ${ }^{1} \mathrm{H}\left(125\right.$ and $\left.500 \mathrm{MHz}, \mathrm{CD}_{3} \mathrm{OD}, 298 \mathrm{~K}\right)$ spectra of $\left[\mathrm{Zn}(\mathbf{T M C}) \mathrm{Cl}_{2}\right]$.
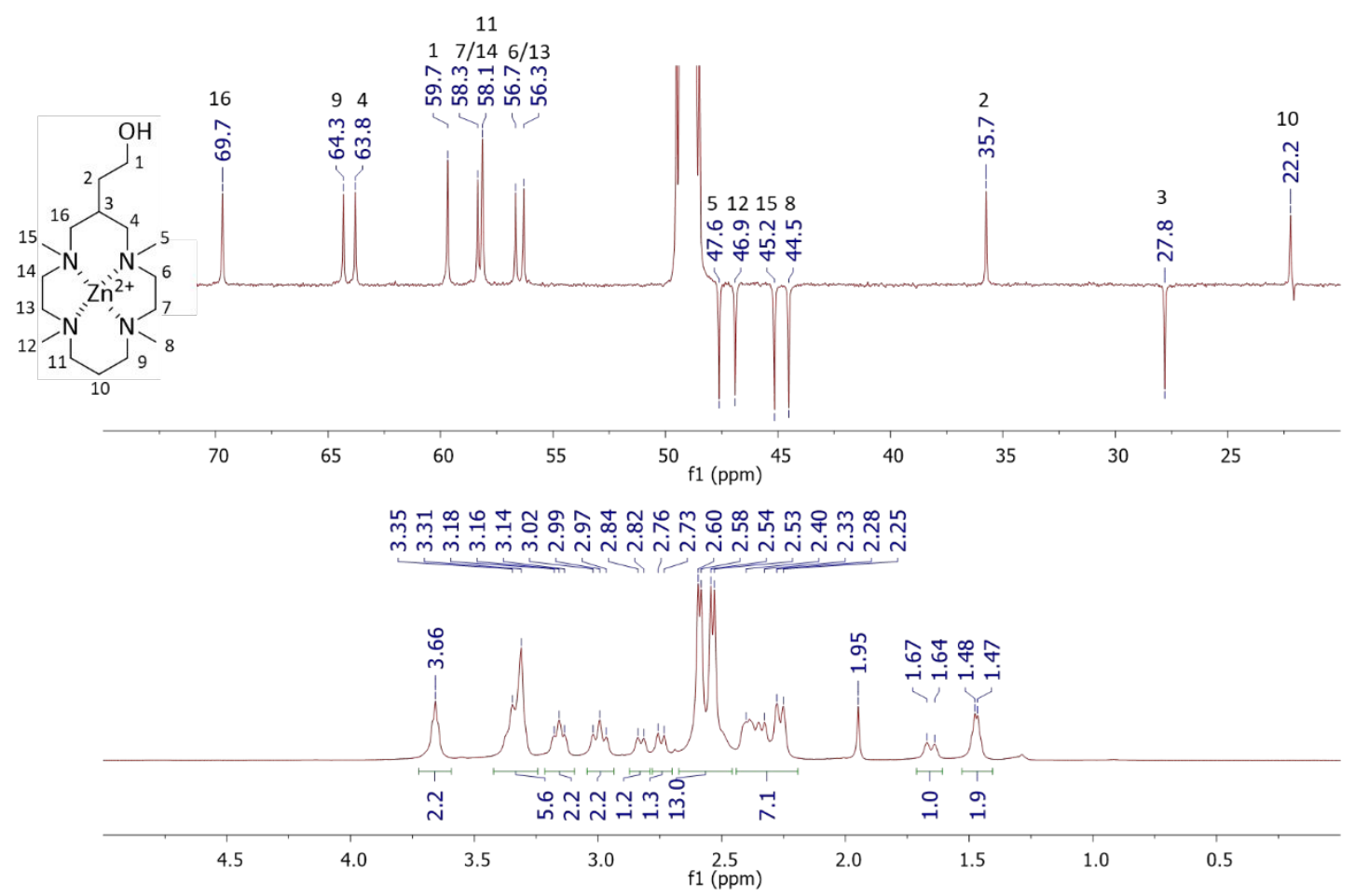

Figure S33. $\mathrm{NMR}{ }^{13} \mathrm{C}$ Jmod and ${ }^{1} \mathrm{H}\left(125\right.$ and $\left.500 \mathrm{MHz}, \mathrm{CD}_{3} \mathrm{OD}, 298 \mathrm{~K}\right)$ spectra of $\left[\mathrm{Zn}(\mathbf{T M C}-\mathbf{E}) \mathrm{Cl}_{2}\right]$. 


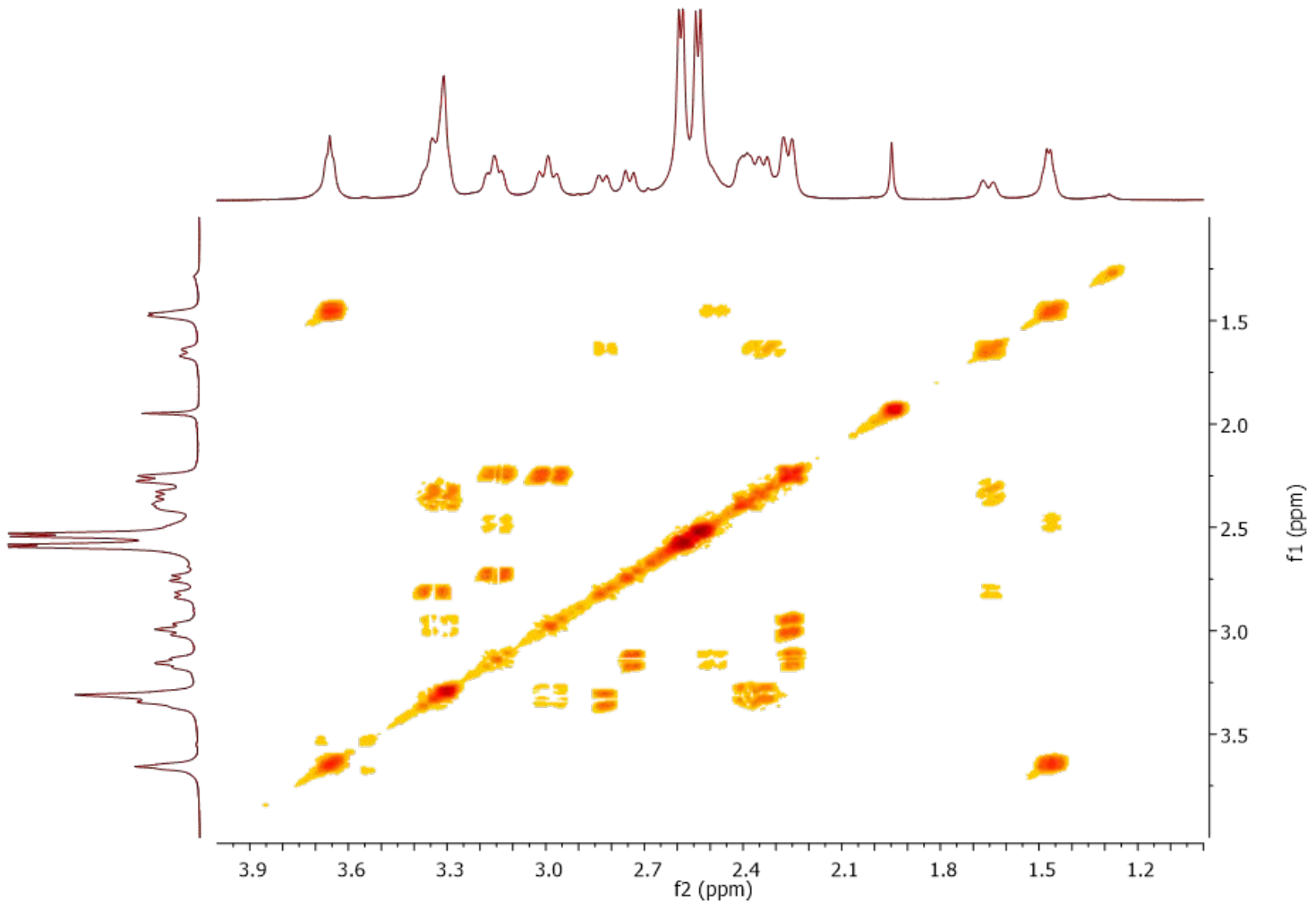

Figure S34. 2D COSY ${ }^{1} \mathrm{H}^{1}{ }^{1} \mathrm{H}$ NMR spectrum $\left(500 \mathrm{MHz}, \mathrm{CD}_{3} \mathrm{OD}, 223 \mathrm{~K}\right)$ of $\left[\mathrm{Zn}(\mathbf{T M C}-\mathbf{E}) \mathrm{Cl}_{2}\right]$.

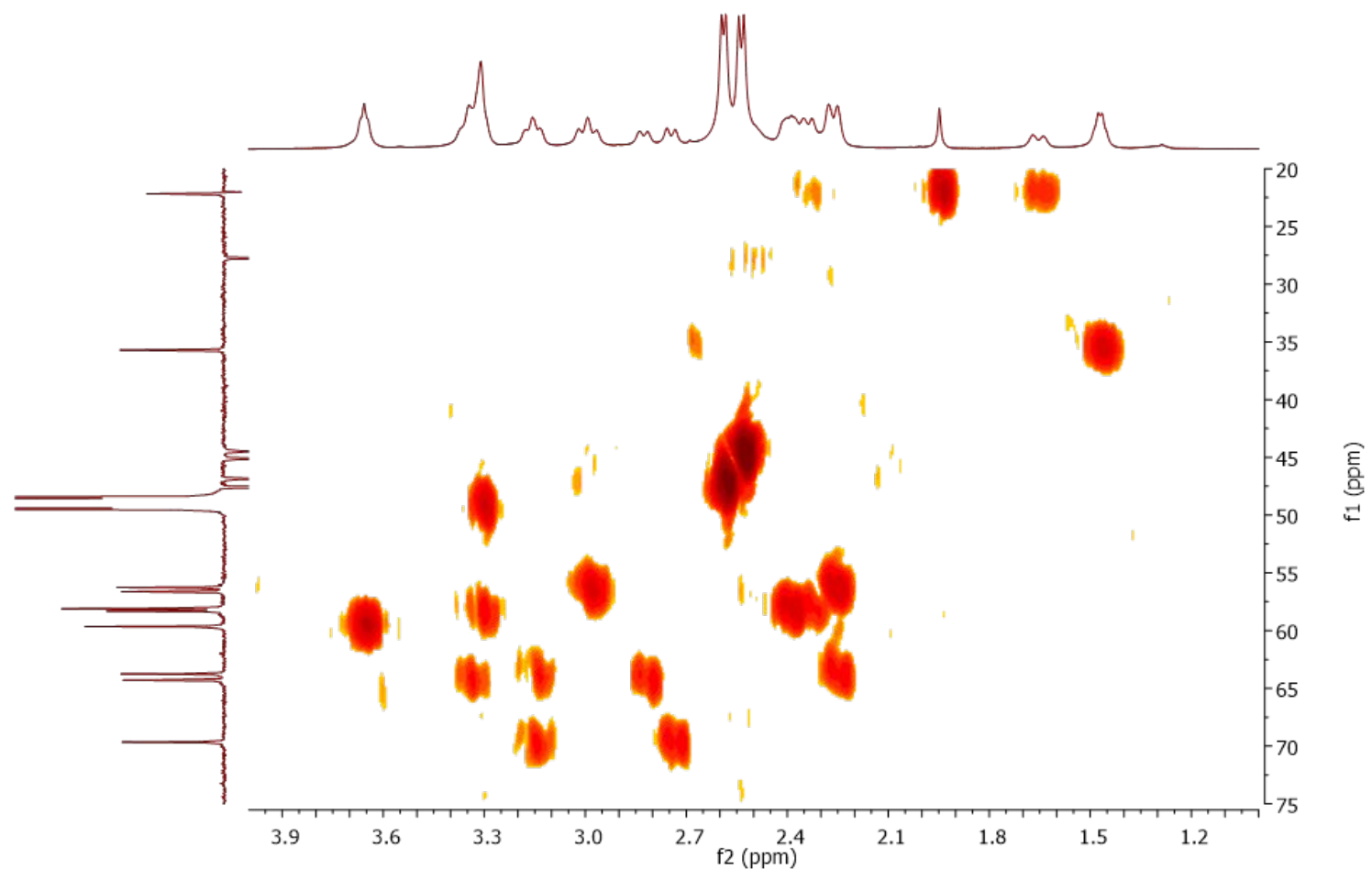

Figure S35. 2D HMQC ${ }^{1} \mathrm{H}_{-}{ }^{13} \mathrm{C}$ NMR spectrum (500 MHz-125 MHz, $\mathrm{CD}_{3} \mathrm{OD}, 223 \mathrm{~K}$ ) of $\left[\mathrm{Zn}(\mathbf{T M C}-\mathbf{E}) \mathrm{Cl}_{2}\right]$. 


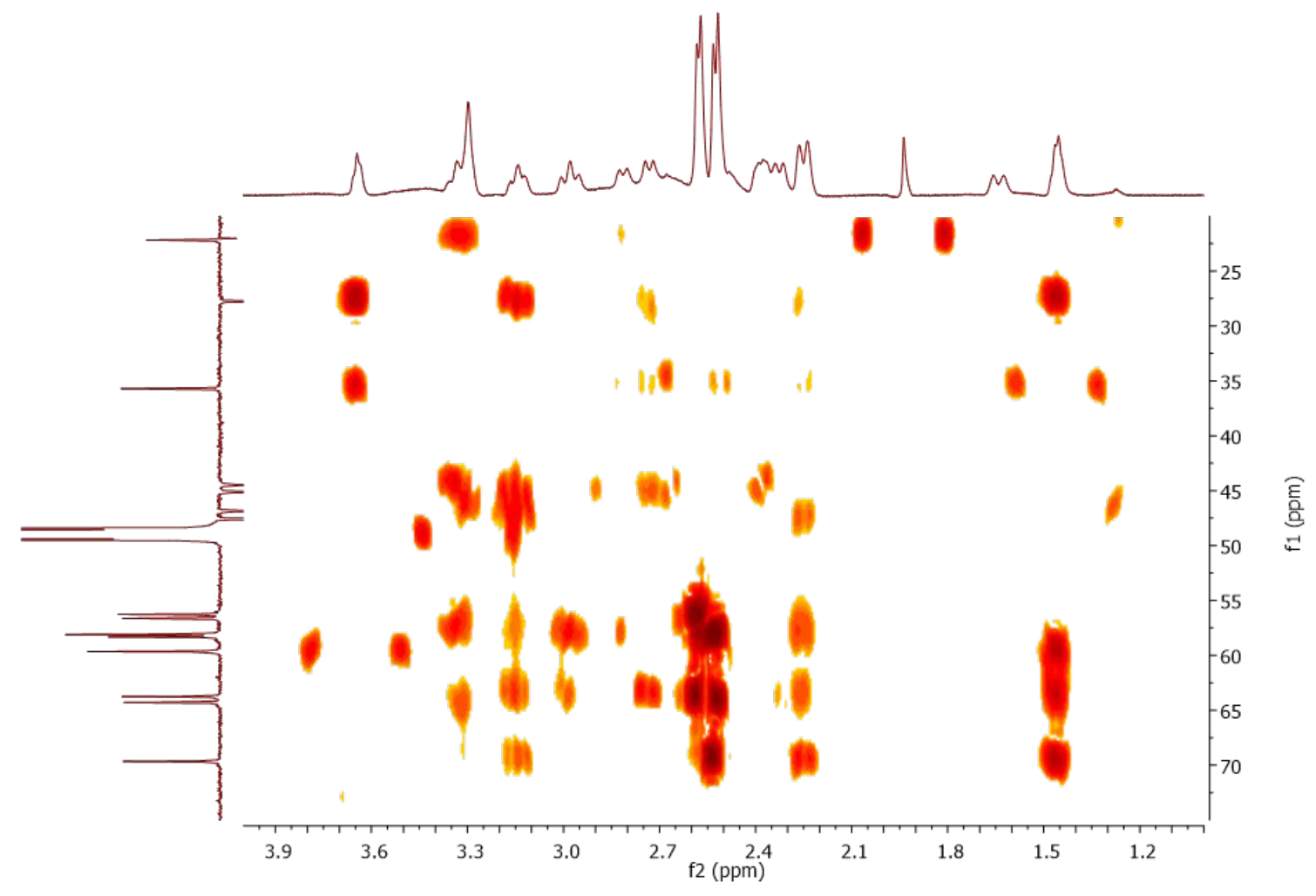

Figure S36. 2D HMBC ${ }^{1} \mathrm{H}_{-13}{ }^{13} \mathrm{C}$ NMR spectrum $\left(500 \mathrm{MHz}-125 \mathrm{MHz}, \mathrm{CD}_{3} \mathrm{OD}, 223 \mathrm{~K}\right)$ of $\left[\mathrm{Zn}(\mathbf{T M C}-\mathbf{E}) \mathrm{Cl}_{2}\right]$.

Table S6. ${ }^{13} \mathrm{C}$ and ${ }^{1} \mathrm{H}$ NMR shifts (ppm) of $\left[\mathrm{Zn}(\mathbf{T M C}-\mathbf{E}) \mathrm{Cl}_{2}\right]$.

\begin{tabular}{|lcc|}
\hline & $\delta^{13} \mathrm{C}(\mathrm{ppm})$ & $\delta^{1} \mathrm{H}$ NMR $(\mathrm{ppm})$ \\
\hline 1 & 59.7 & 3.66 \\
2 & 35.7 & $1.48-1.47$ \\
3 & 27.8 & $2.53-2.49$ \\
4 & 63.8 & $3.18-3.14 / 2.28-2.25$ \\
6 & 47.6 & 2.60 or 2.58 \\
7 & 56.7 or 56.3 & $3.02-2.97 / 2.28-2.25$ \\
8 & 58.1 or 58.3 & $3.38-3.31 / 2.40-2.33$ \\
9 & 44.5 & 2.54 or 2.53 \\
10 & 58.1 & $3.38-3.31 / 2.40-2.33$ \\
11 & 22.2 & $2.40-2.33 / 1.67-1.64$ \\
12 & 64.3 & $3.38-3.31 / 2.84-2.84$ \\
13 & 46.9 & 2.60 or 2.58 \\
14 & 56.7 or 56.3 & $3.02-2.97 / 2.28-2.25$ \\
15 & 58.1 or 58.3 & $3.38-3.31 / 2.40-2.33$ \\
16 & 45.2 & 2.54 or 2.53 \\
\hline
\end{tabular}

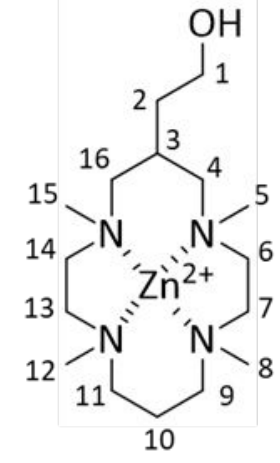



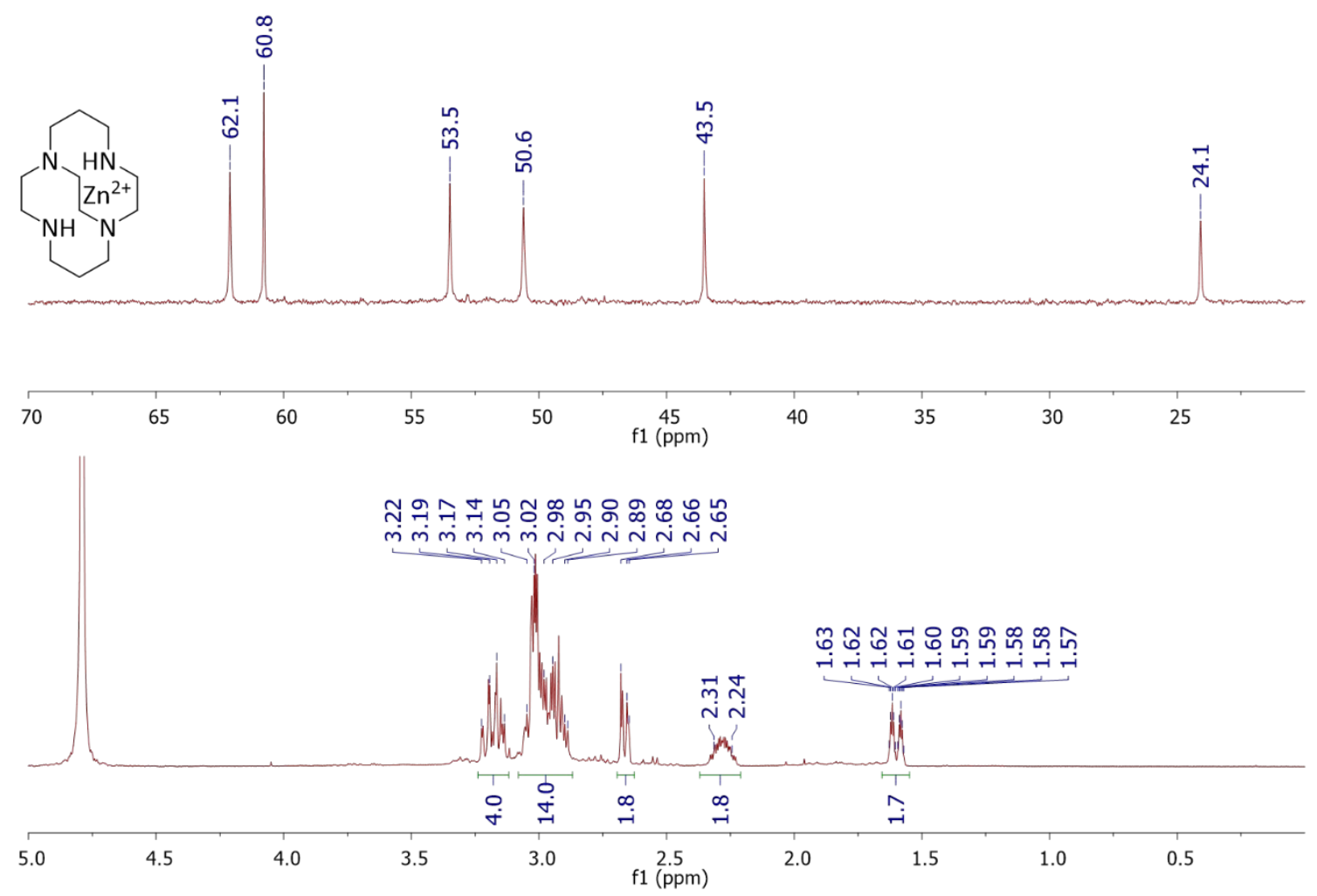

Figure S37. NMR ${ }^{13} \mathrm{C}$ Jmod and ${ }^{1} \mathrm{H}\left(125\right.$ and $\left.500 \mathrm{MHz}, \mathrm{D}_{2} \mathrm{O}, 298 \mathrm{~K}\right)$ spectra of $\left[\mathrm{Zn}(\mathbf{C B}-\right.$ cyclam $\left.) \mathrm{Cl}_{2}\right]$.

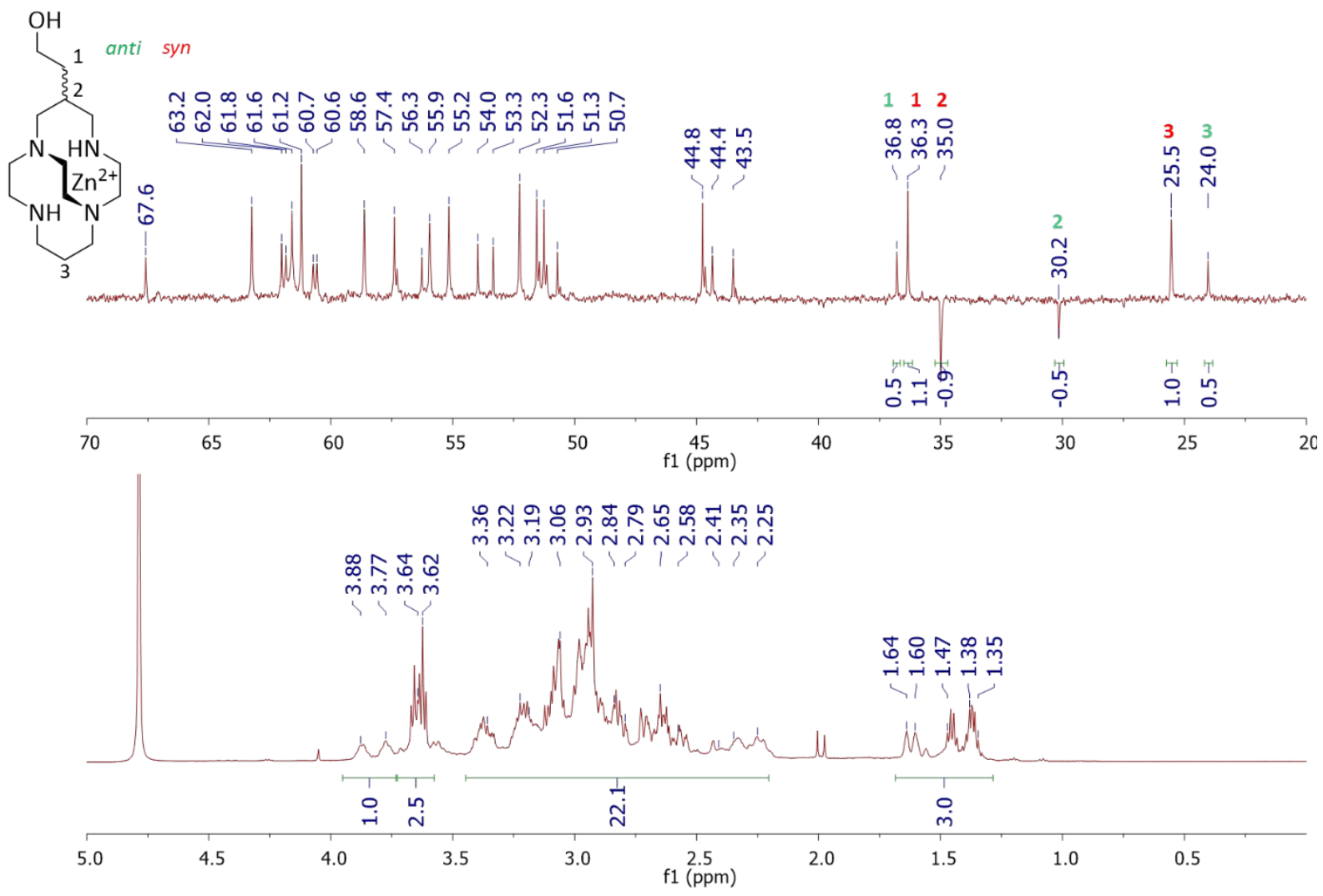

Figure S38. $\mathrm{NMR}{ }^{13} \mathrm{C}$ Jmod and ${ }^{1} \mathrm{H}\left(125\right.$ and $\left.500 \mathrm{MHz}, \mathrm{D}_{2} \mathrm{O}, 298 \mathrm{~K}\right)$ spectra of $[\mathrm{Zn}(\mathbf{C B}-$ cyclam-E) $\mathrm{Cl}_{2}$. 

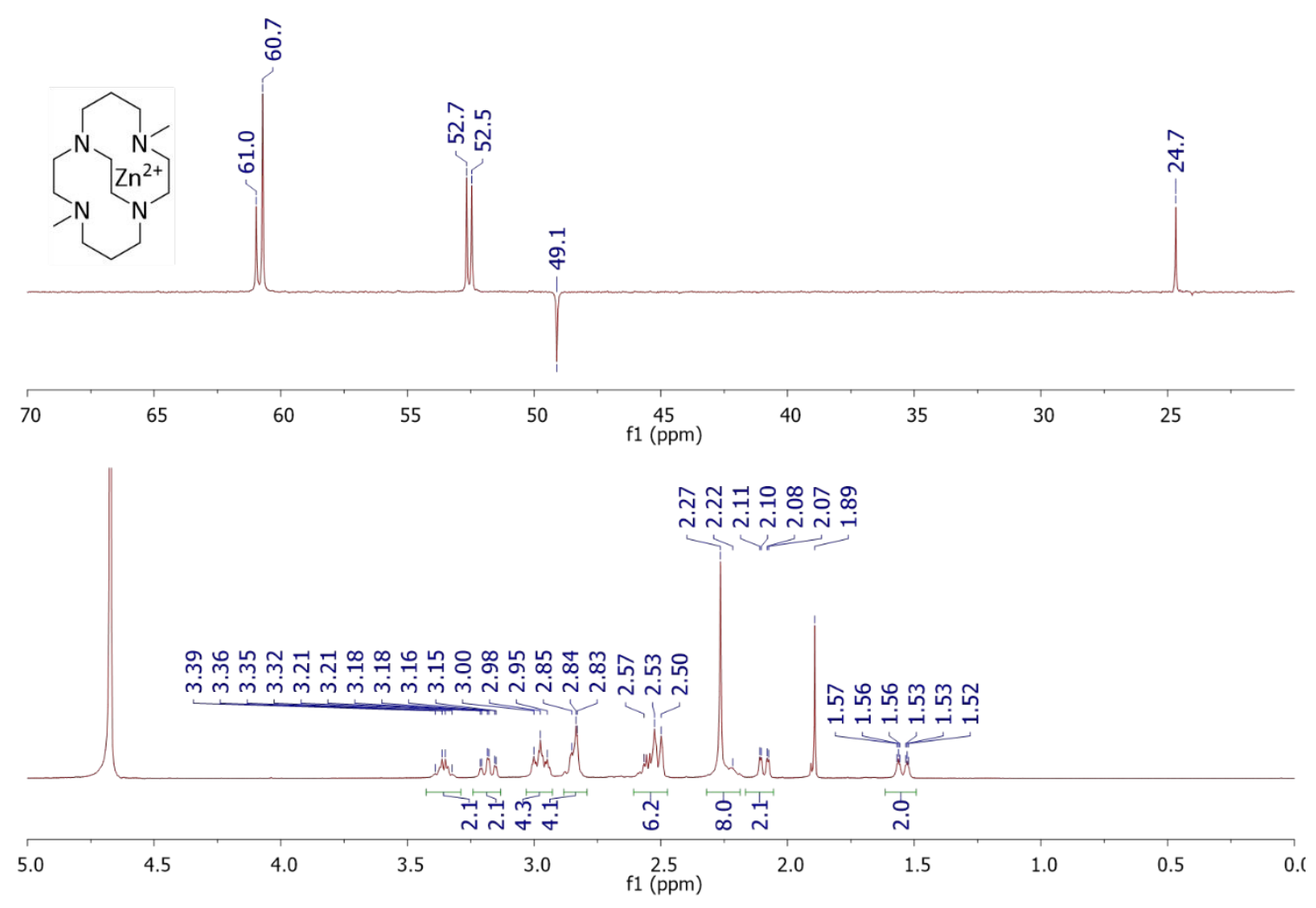

Figure S39. NMR ${ }^{13} \mathrm{C}$ Jmod and ${ }^{1} \mathrm{H}\left(125\right.$ and $\left.500 \mathrm{MHz}, \mathrm{D}_{2} \mathrm{O}, 298 \mathrm{~K}\right)$ spectra of [Zn(CB-DMC $) \mathrm{Cl}_{2}$ ].
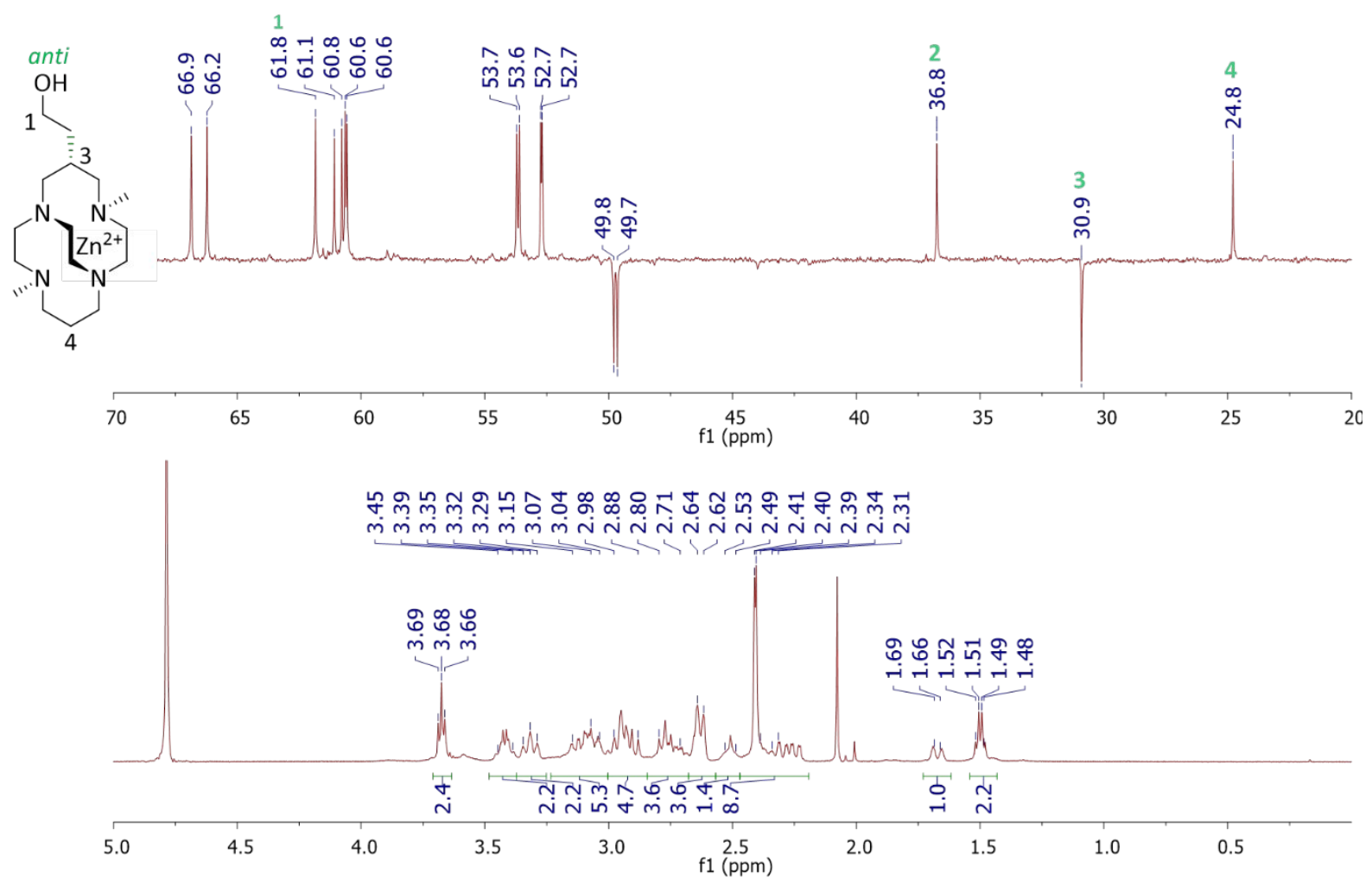

Figure S40. NMR ${ }^{13} \mathrm{C}$ Jmod and ${ }^{1} \mathrm{H}\left(125\right.$ and $\left.500 \mathrm{MHz}, \mathrm{D}_{2} \mathrm{O}, 298 \mathrm{~K}\right)$ spectra of anti-[Zn(CB-DMC-E) $\left.\mathrm{Cl}_{2}\right]$. 

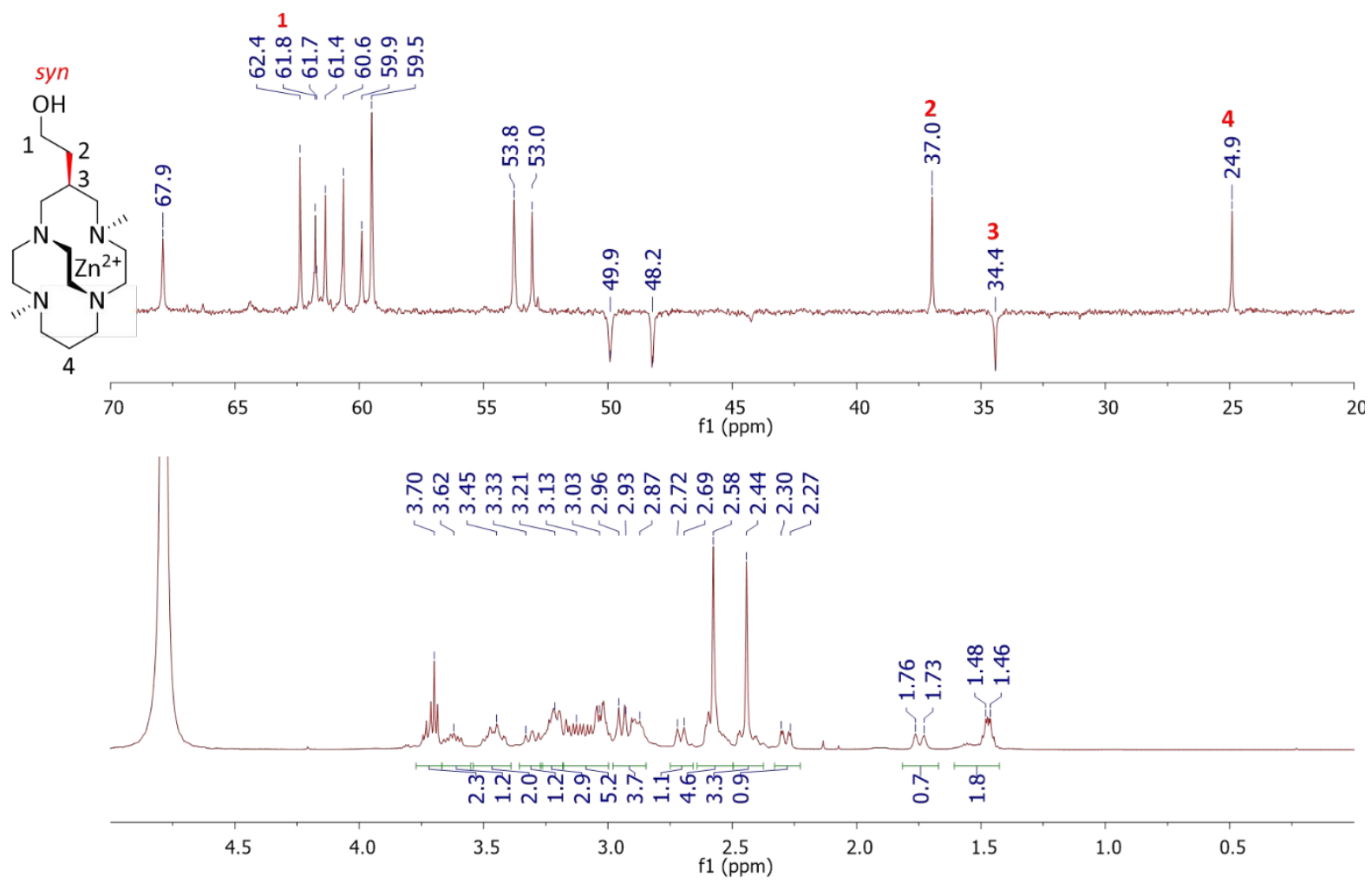

Figure S41. NMR ${ }^{13} \mathrm{C}$ Jmod and ${ }^{1} \mathrm{H}\left(125\right.$ and $\left.500 \mathrm{MHz}, \mathrm{D}_{2} \mathrm{O}, 298 \mathrm{~K}\right)$ spectra of syn-[Zn(CB-DMC-E) $\left.\mathrm{Cl}_{2}\right]$.

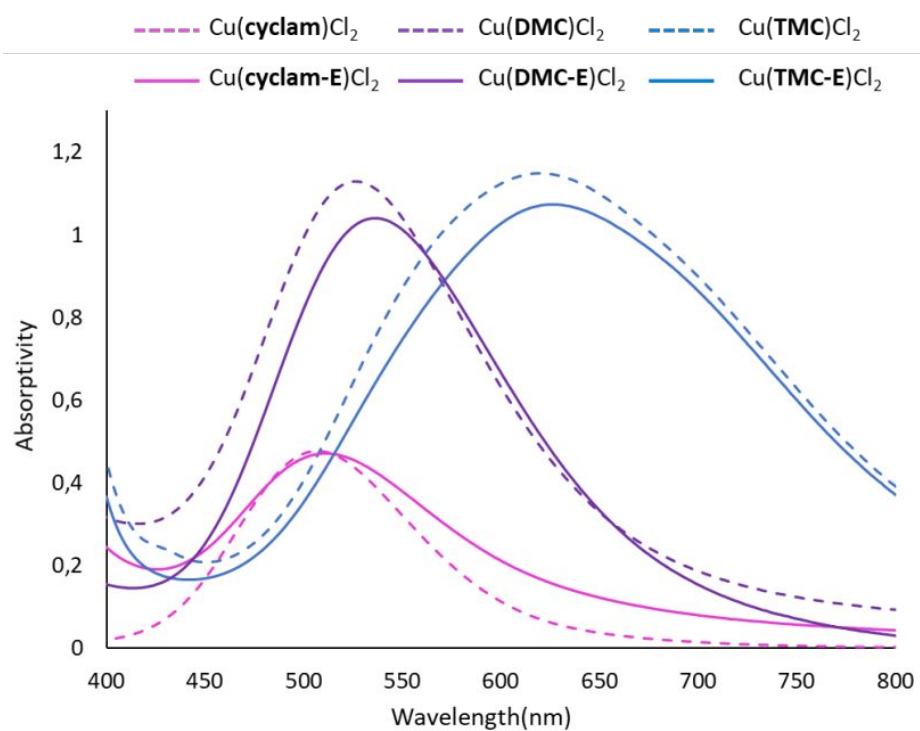

Figure S42. Visible spectra of $\left[\mathrm{Cu}\left(\right.\right.$ cyclam) $\left.\mathrm{Cl}_{2}\right]$ (pink dashed), $\left[\mathrm{Cu}\left(\right.\right.$ cyclam-E) $\left.\mathrm{Cl}_{2}\right]$ (pink), $\left[\mathrm{Cu}(\mathbf{D M C}) \mathrm{Cl}_{2}\right]$ (purple dashed), [Cu(DMC-E)Cl $\left.{ }_{2}\right]$ (purple), [Cu(TMC)Cl$\left.{ }_{2}\right]$ (blue dashed) and $\left[\mathrm{Cu}(\mathbf{T M C}-\mathbf{E}) \mathrm{Cl}_{2}\right]$ (blue) in aqueous solution $(\mathrm{pH} 7 . \mathrm{x})$ at $25^{\circ} \mathrm{C}$. 


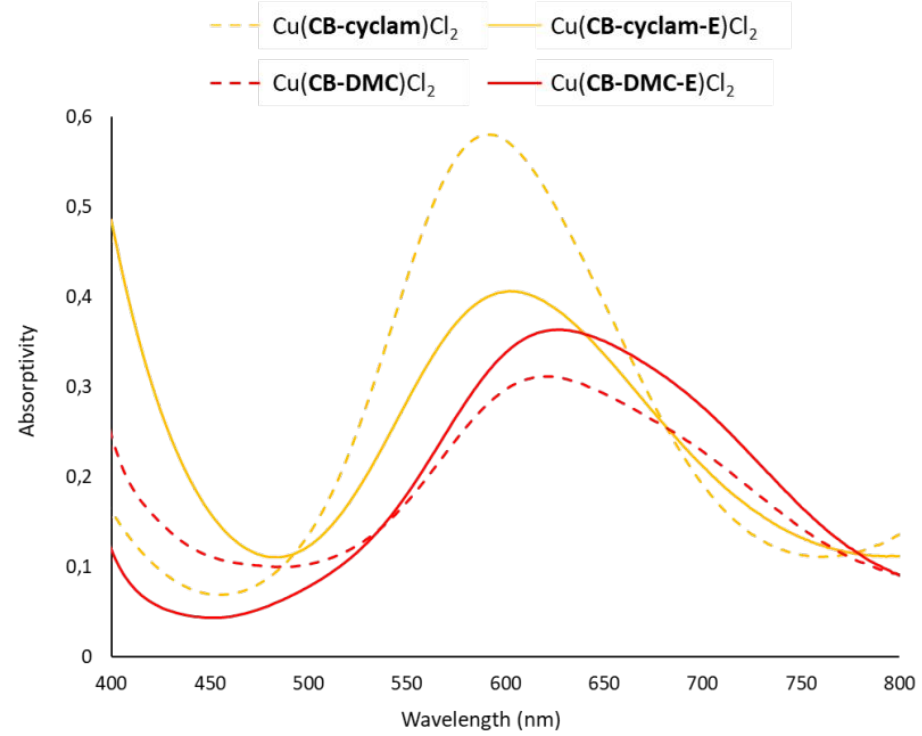

Figure 43. Visible spectra of $\left[\mathrm{Cu}(\mathbf{C B}-\right.$ cyclam $\left.) \mathrm{Cl}_{2}\right]$ (yellow dashed), $\left.\mathrm{Cu}(\mathbf{C B}-\mathbf{c y c l a m}-\mathbf{E}) \mathrm{Cl}_{2}\right]$ (yellow), $\left[\mathrm{Cu}(\mathbf{C B}-\mathbf{D M C}) \mathrm{Cl}_{2}\right]$ (red dashed) and $\left[\mathrm{Cu}(\mathbf{C B}-\mathbf{D M C}-\mathbf{E}) \mathrm{Cl}_{2}\right]$ (red) in aqueous solution (pH 7.x).

\section{Table S7}

BH\&HLYP functional of $g$ - and $A$-values 

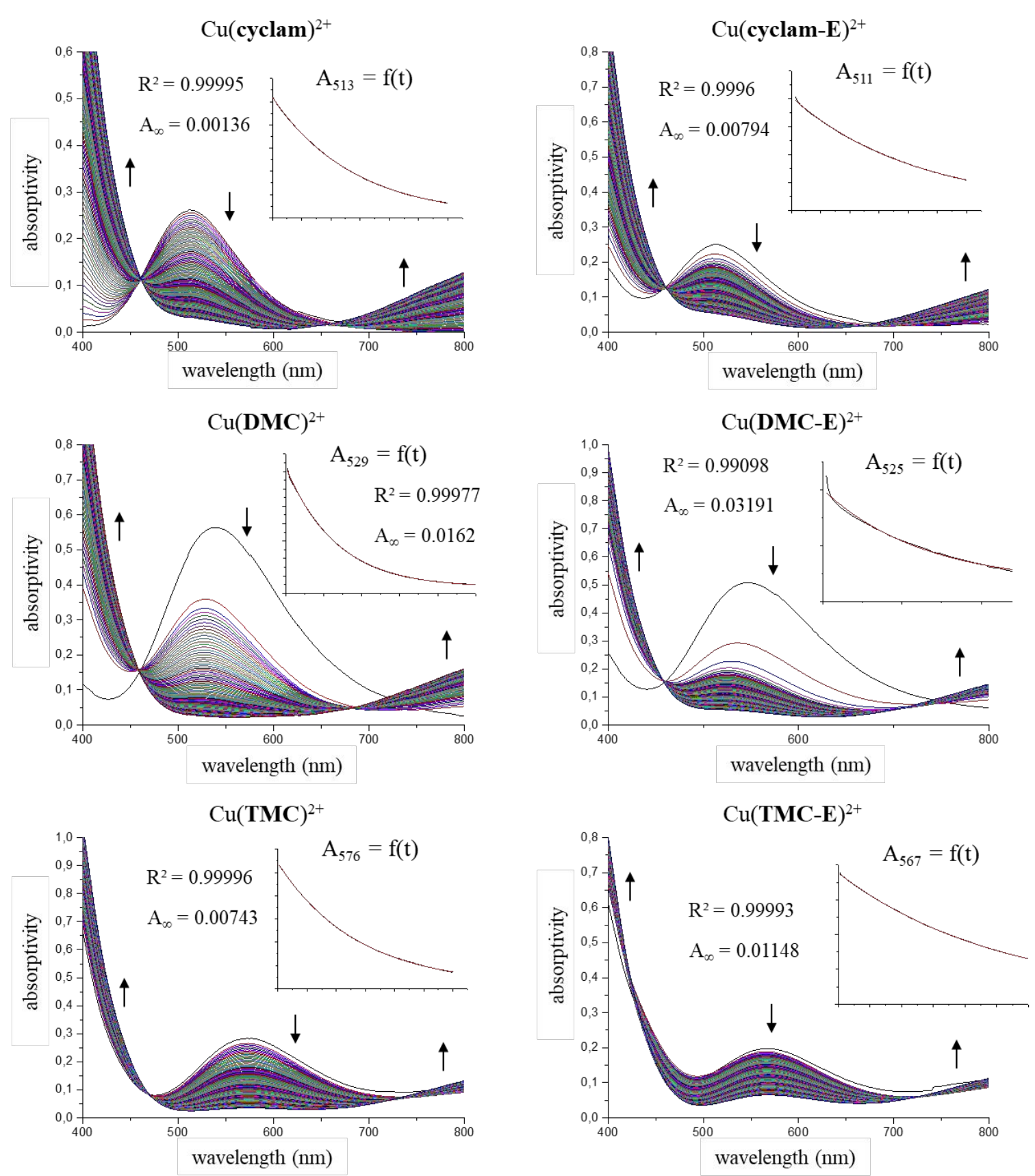

Figure S44. Visible spectra of acid-mediated dissociation of $\mathrm{Cu}(\mathbf{c y c l a m})^{2+}, \mathrm{Cu}(\mathbf{c y c l a m}-\mathrm{E})^{2+}$, $\mathrm{Cu}(\mathbf{D M C})^{2+}, \mathrm{Cu}(\mathbf{D M C}-\mathbf{E})^{2+}$, trans-III-Cu(TMC) $)^{2+}$ and trans-III-Cu(TMC-E) $)^{2+}$ at $2.85 \mathrm{mM}$ in $5 \mathrm{M} \mathrm{HCl}$ at $50{ }^{\circ} \mathrm{C}$ with $10 \mathrm{~min}$ gap. Arrows indicate the changes in absorbance during the experiment on both sides of isosbestic points. Exponential curves corresponding to experimental/model fitting of the function $A_{\lambda \max }=f(t)$ made on OriginPro9. $R^{2}$ is the adjusting parameter and $\mathrm{A}_{\infty}$ the asymptote of the exponential model. The first curve of each experiment was excluded due to fast changes of absorbance between 0 and 10 min following $\mathrm{HCl}$ addition. 

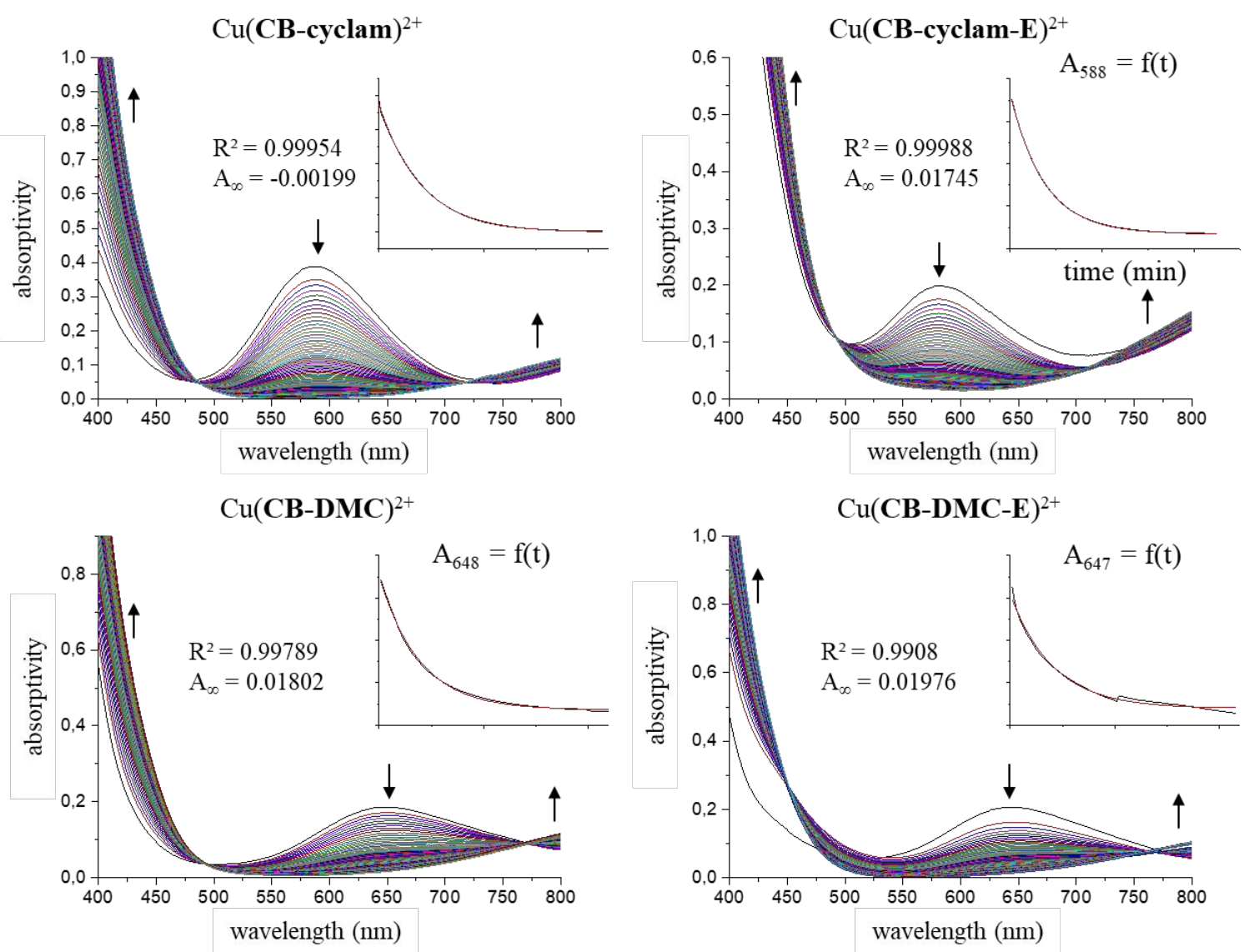

Figure S45. UV-Visible spectra of acid-mediated dissociation of $\mathrm{Cu}(\mathbf{C B}-\mathbf{c y c l a m})^{2+}, \mathrm{Cu}(\mathbf{C B}-$ cyclam-E $)^{2+}, \mathrm{Cu}(\mathbf{C B}-\mathbf{D M C})^{2+}$ and $\mathrm{Cu}(\mathbf{C B}-\mathbf{D M C}-\mathbf{E})^{2+}$, at $2.85 \mathrm{mM}$ in $5 \mathrm{M} \mathrm{HCl}$ at $70{ }^{\circ} \mathrm{C}$ with 10 min gap. Arrows indicate the changes in absorbance during the experiment on both sides of isosbestic points. Exponential curves corresponding to experimental/model fitting of the function $A_{\lambda \max }=f(t)$ made on OriginPro9. $R^{2}$ is the adjusting parameter and $A_{\infty}$ the asymptote of the exponential model.

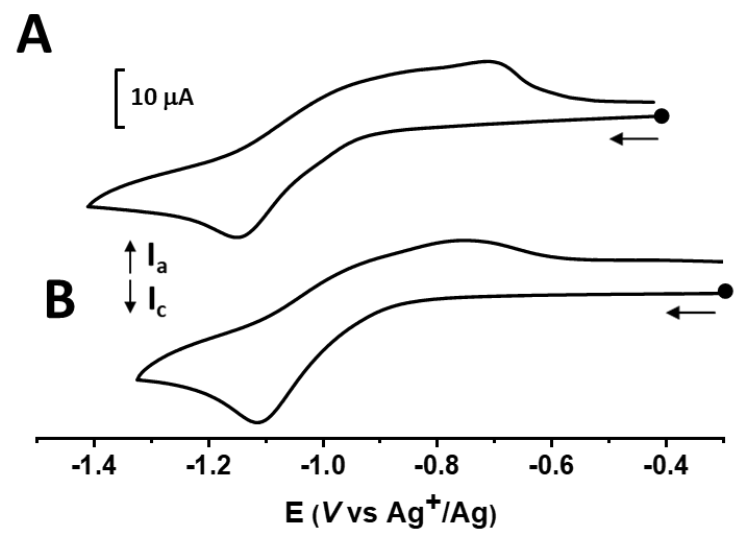

Figure 46. Cyclic voltammograms of (A) $\mathrm{Cu}(\mathbf{D M C})^{2+}$ and (B) $\mathrm{Cu}(\mathbf{D M C}-\mathbf{E})^{2+}$ in $\mathrm{CH}_{3} \mathrm{CN}+$ $\mathrm{TBAPF}_{6}(0.1 \mathrm{M})$. Scan rate $0.1 \mathrm{~V} \cdot \mathrm{s}^{-1}$. 

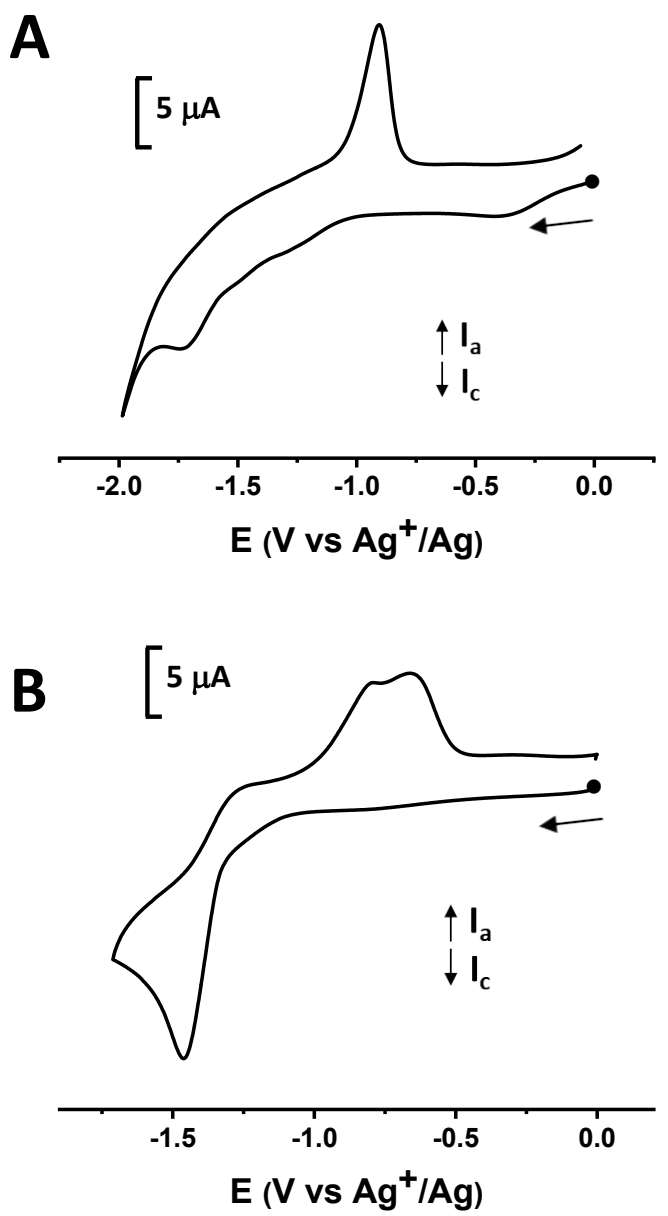

Figure 47. Cyclic voltammograms of $(\mathrm{A}) \mathrm{Cu}(\text { cyclam-E })^{2+}$ in $\mathrm{DMF}+\mathrm{TBACl}(0.1 \mathrm{M})$ and (B) $\mathrm{Cu}(\text { cyclam })^{2+}$ in $\mathrm{CH}_{3} \mathrm{CN}+\mathrm{TBAPF}_{6}(0.1 \mathrm{M})$. Scan rate $0.1{\mathrm{~V} . \mathrm{s}^{-1}}^{-}$. 
Table S7. Cartesian coordinates $(\AA)$ of $[\mathrm{Cu}(\text { cyclam }) \mathrm{Cl}]^{+}$(trans-III) optimized at the uTPSSh/Def2-TZVPP level (scrf=pcm).

40

\section{$\mathrm{Cu}($ cyclam $) \mathrm{Cl}$}

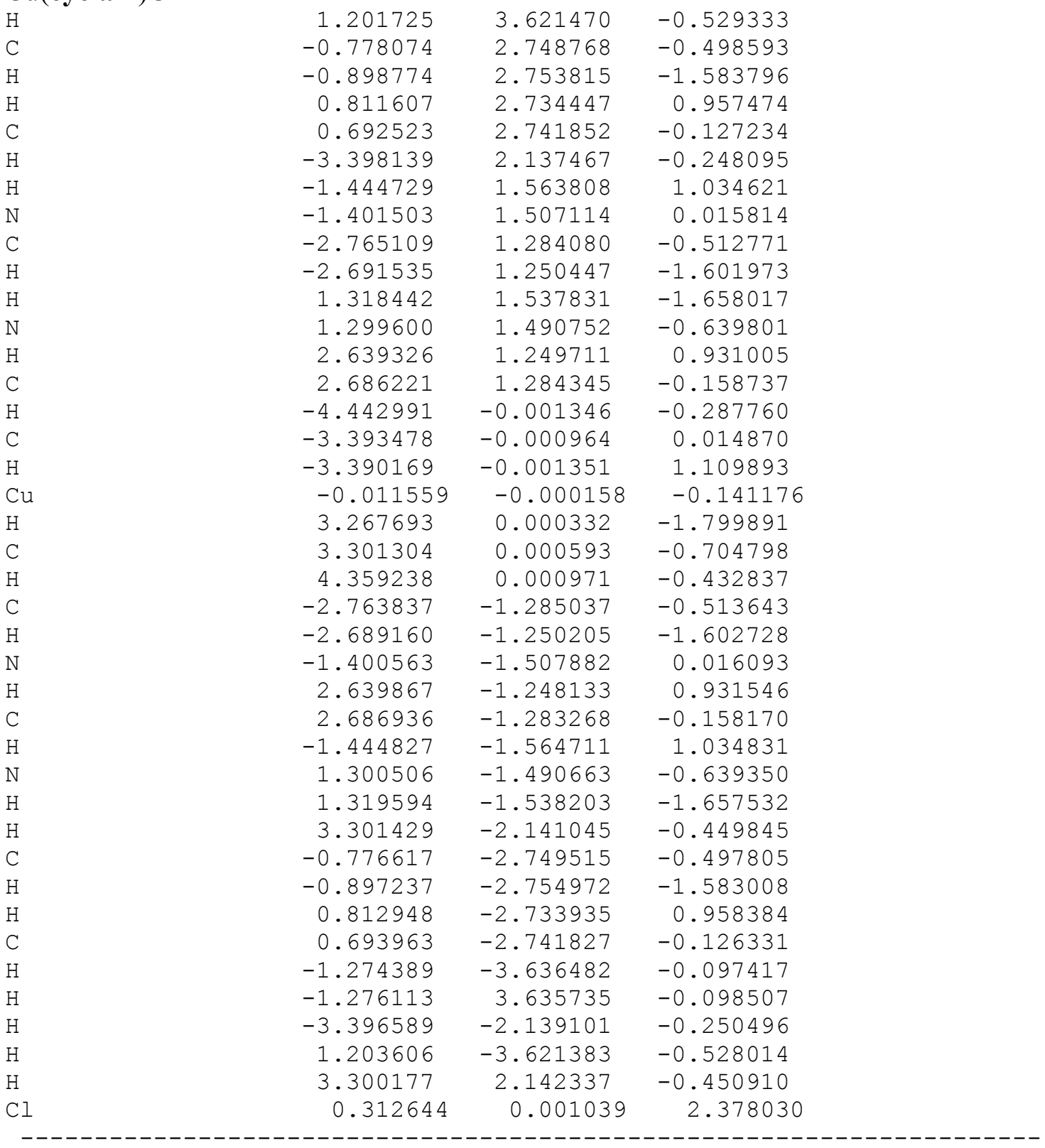

$\mathrm{E}(\mathrm{UTPSSh})=-2715.5433105$ Hartree

Zero-point correction $=0.361611$

Thermal correction to Energy $=0.378817$

Thermal correction to Enthalpy $=0.379761$

Thermal correction to Gibbs Free Energy $=0.317013$

Sum of electronic and zero-point Energies $=-2715.181700$

Sum of electronic and thermal Energies $=-2715.164494$

Sum of electronic and thermal Enthalpies $=-2715.163549$

Sum of electronic and thermal Free Energies = -2715.226298 
Table S8. Cartesian coordinates $(\AA)$ of $\left[\mathrm{Cu}(\text { cyclam })\left(\mathrm{H}_{2} \mathrm{O}\right)\right]^{2+}$ (trans-III) optimized at the uTPSSh/Def2-TZVPP level (scrf=pcm).

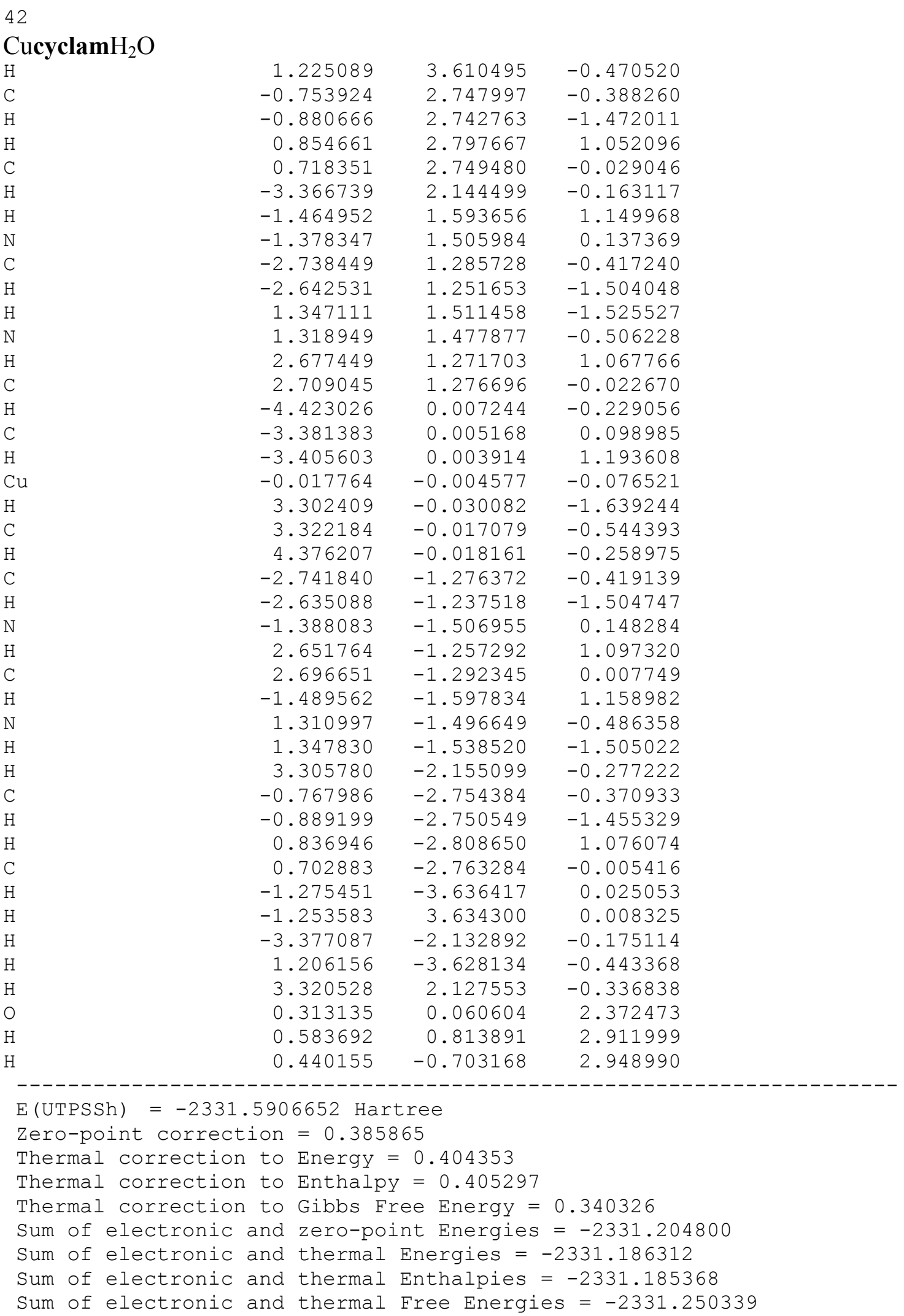


Table S9. Cartesian coordinates $(\AA)$ of $\left[\mathrm{Cu}(\text { cyclam-E) })\left(\mathrm{H}_{2} \mathrm{O}\right)\right]^{2+}$ (trans-III) optimized at the uTPSSh/Def2-TZVPP level (scrf=pcm).

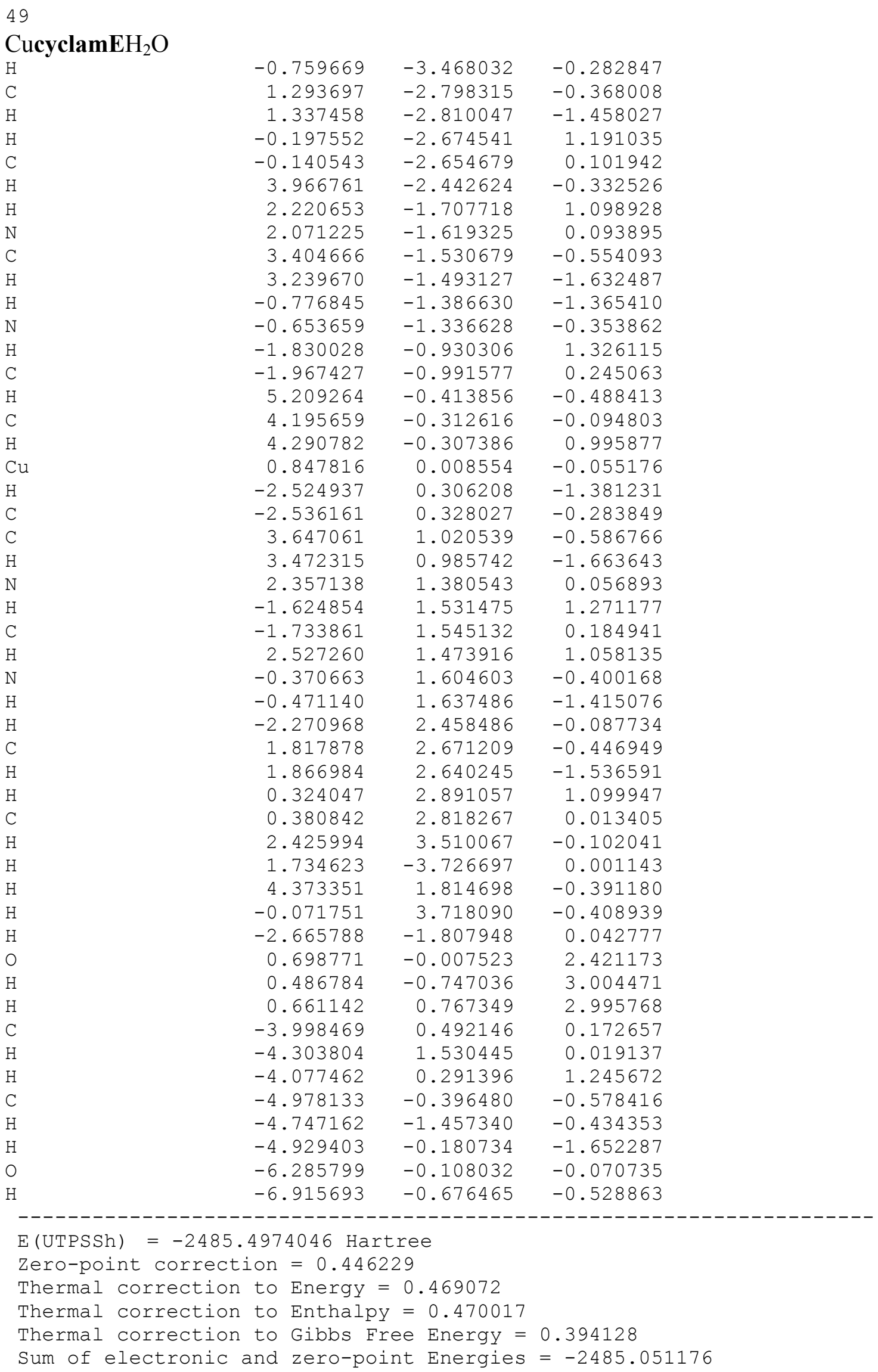


Sum of electronic and thermal Energies $=-2485.028332$

Sum of electronic and thermal Enthalpies $=-2485.027388$

Sum of electronic and thermal Free Energies $=-2485.103276$

Table S10. Cartesian coordinates $(\AA)$ of $\left[\mathrm{Cu}(\mathbf{D M C})\left(\mathrm{H}_{2} \mathrm{O}\right)\right]^{2+}$ (trans-III) optimized at the uTPSSh/Def2-TZVPP level (scrf=pcm).

48

\section{$\mathrm{CuDMCH}{ }_{2} \mathrm{O}$}

\begin{tabular}{|c|c|c|c|}
\hline $\mathrm{H}$ & 2.410179 & 2.957651 & 0.234194 \\
\hline C & 0.257864 & 2.864455 & 0.091931 \\
\hline $\mathrm{H}$ & 0.231285 & 3.162738 & -0.955247 \\
\hline $\mathrm{H}$ & 1.622754 & 2.037314 & 1.528386 \\
\hline $\mathrm{C}$ & 1.596972 & 2.261703 & 0.462253 \\
\hline $\mathrm{H}$ & -2.385546 & 3.194396 & -0.124498 \\
\hline $\mathrm{H}$ & -1.035583 & 1.838345 & 1.291058 \\
\hline $\mathrm{N}$ & -0.812592 & 1.849471 & 0.296619 \\
\hline C & -2.046723 & 2.207318 & -0.452579 \\
\hline $\mathrm{H}$ & -1.771298 & 2.283693 & -1.505740 \\
\hline $\mathrm{N}$ & 1.799532 & 0.976891 & -0.265874 \\
\hline $\mathrm{H}$ & 2.806747 & 0.237022 & 1.408641 \\
\hline C & 2.961363 & 0.255453 & 0.329810 \\
\hline $\mathrm{H}$ & -4.037655 & 1.586020 & -0.809336 \\
\hline C & -3.168829 & 1.196373 & -0.273752 \\
\hline $\mathrm{H}$ & -3.469922 & 1.137852 & 0.774817 \\
\hline $\mathrm{Cu}$ & -0.016793 & -0.005107 & -0.026520 \\
\hline $\mathrm{H}$ & 3.383623 & -1.162245 & -1.261769 \\
\hline C & 3.176856 & -1.162460 & -0.188639 \\
\hline $\mathrm{H}$ & 4.087706 & -1.532595 & 0.287285 \\
\hline C & -2.882809 & -0.185348 & -0.850598 \\
\hline $\mathrm{H}$ & -2.521284 & -0.088677 & -1.875864 \\
\hline $\mathrm{N}$ & -1.864306 & -0.978136 & -0.098962 \\
\hline $\mathrm{H}$ & 1.861204 & -2.192192 & 1.197440 \\
\hline C & 2.064443 & -2.152776 & 0.126425 \\
\hline $\mathrm{N}$ & 0.791070 & -1.796446 & -0.551534 \\
\hline $\mathrm{H}$ & 0.981534 & -1.723112 & -1.550642 \\
\hline $\mathrm{H}$ & 2.368372 & -3.153959 & -0.192828 \\
\hline $\mathrm{C}$ & -1.576381 & -2.238290 & -0.846983 \\
\hline $\mathrm{H}$ & -1.514587 & -1.986627 & -1.906833 \\
\hline $\mathrm{H}$ & -0.291346 & -3.102334 & 0.673003 \\
\hline $\mathrm{C}$ & -0.262000 & -2.828846 & -0.381188 \\
\hline $\mathrm{H}$ & -2.396083 & -2.949905 & -0.714405 \\
\hline $\mathrm{H}$ & 0.063763 & 3.754322 & 0.693679 \\
\hline $\mathrm{H}$ & -3.810105 & -0.768410 & -0.877166 \\
\hline $\mathrm{H}$ & -0.020453 & -3.726675 & -0.954188 \\
\hline $\mathrm{H}$ & 3.862626 & 0.849227 & 0.140310 \\
\hline $\mathrm{C}$ & -2.387823 & -1.322946 & 1.246715 \\
\hline $\mathrm{H}$ & -1.673579 & -1.949827 & 1.773705 \\
\hline $\mathrm{H}$ & -2.560957 & -0.420923 & 1.827169 \\
\hline $\mathrm{H}$ & -3.334037 & -1.863174 & 1.143633 \\
\hline C & 2.047318 & 1.238979 & -1.706903 \\
\hline $\mathrm{H}$ & 2.931828 & 1.873063 & -1.821176 \\
\hline $\mathrm{H}$ & 2.213159 & 0.303270 & -2.233572 \\
\hline $\mathrm{H}$ & 1.188968 & 1.733587 & -2.153957 \\
\hline O & 0.315081 & -0.099105 & 2.460072 \\
\hline $\mathrm{H}$ & 1.104007 & -0.342649 & 2.959813 \\
\hline $\mathrm{H}$ & -0.406421 & -0.208410 & 3.091904 \\
\hline
\end{tabular}


Thermal correction to Energy $=0.461861$

Thermal correction to Enthalpy $=0.462805$

Thermal correction to Gibbs Free Energy $=0.392805$

Sum of electronic and zero-point Energies $=-2409.783033$

Sum of electronic and thermal Energies $=-2409.761797$

Sum of electronic and thermal Enthalpies $=-2409.760853$

Sum of electronic and thermal Free Energies $=-2409.830852$

Table S11. Cartesian coordinates $(\AA)$ of $\left[\mathrm{Cu}(\mathbf{D M C}-\mathbf{E})\left(\mathrm{H}_{2} \mathrm{O}\right)\right]^{2+}$ (trans-III) optimized at the uTPSSh/Def2-TZVPP level (scrf=pcm).

55

\section{$\mathrm{CuDMCEH}_{2} \mathrm{O}$}

\begin{tabular}{|c|c|c|c|}
\hline $\mathrm{H}$ & 0.987550 & 3.420293 & 0.188575 \\
\hline C & -1.102431 & 2.882091 & 0.109772 \\
\hline $\mathrm{H}$ & -1.223225 & 3.176143 & -0.931972 \\
\hline $\mathrm{H}$ & 0.449358 & 2.355898 & 1.497593 \\
\hline C & 0.344138 & 2.570123 & 0.434341 \\
\hline $\mathrm{H}$ & -3.763206 & 2.657123 & -0.018359 \\
\hline $\mathrm{H}$ & -2.103959 & 1.588222 & 1.331960 \\
\hline N & -1.931388 & 1.665310 & 0.330090 \\
\hline C & -3.237717 & 1.765677 & -0.372667 \\
\hline H & -3.018321 & 1.907048 & -1.432408 \\
\hline $\mathrm{N}$ & 0.789002 & 1.356566 & -0.311083 \\
\hline $\mathrm{H}$ & 1.937182 & 0.791734 & 1.342682 \\
\hline $\mathrm{C}$ & 2.080206 & 0.882882 & 0.265165 \\
\hline $\mathrm{H}$ & -5.066056 & 0.747019 & -0.682246 \\
\hline C & -4.118226 & 0.540653 & -0.179886 \\
\hline $\mathrm{H}$ & -4.366557 & 0.405707 & 0.875299 \\
\hline $\mathrm{Cu}$ & -0.782120 & 0.027396 & -0.052576 \\
\hline $\mathrm{H}$ & 2.718476 & -0.381932 & -1.371179 \\
\hline C & 2.611705 & -0.445304 & -0.283138 \\
\hline $\mathrm{C}$ & -3.567933 & -0.741930 & -0.792918 \\
\hline $\mathrm{H}$ & -3.266423 & -0.554874 & -1.825109 \\
\hline $\mathrm{N}$ & -2.381502 & -1.311047 & -0.086079 \\
\hline $\mathrm{H}$ & 1.537258 & -1.714601 & 1.115599 \\
\hline $\mathrm{C}$ & 1.706863 & -1.634591 & 0.040438 \\
\hline N & 0.376746 & -1.541749 & -0.613742 \\
\hline $\mathrm{H}$ & 0.532510 & -1.421759 & -1.614639 \\
\hline $\mathrm{H}$ & 2.196664 & -2.554837 & -0.291356 \\
\hline C & -1.854269 & -2.471291 & -0.864546 \\
\hline $\mathrm{H}$ & -1.875559 & -2.197713 & -1.920544 \\
\hline $\mathrm{H}$ & -0.378041 & -3.061003 & 0.611288 \\
\hline C & -0.432888 & -2.775424 & -0.438717 \\
\hline $\mathrm{H}$ & -2.500114 & -3.343068 & -0.726520 \\
\hline $\mathrm{H}$ & -1.458351 & 3.707968 & 0.728663 \\
\hline $\mathrm{H}$ & -4.352335 & -1.506820 & -0.806483 \\
\hline H & -0.021882 & -3.595688 & -1.030796 \\
\hline H & 2.822396 & 1.671475 & 0.102077 \\
\hline C & -2.773317 & -1.771842 & 1.269474 \\
\hline $\mathrm{H}$ & -1.922828 & -2.233160 & 1.764536 \\
\hline $\mathrm{H}$ & -3.118214 & -0.932423 & 1.867427 \\
\hline $\mathrm{H}$ & -3.582537 & -2.503878 & 1.188779 \\
\hline $\mathrm{C}$ & 0.959267 & 1.678629 & -1.751518 \\
\hline $\mathrm{H}$ & 1.737045 & 2.438831 & -1.871652 \\
\hline H & 1.245142 & 0.787456 & -2.303639 \\
\hline $\mathrm{H}$ & 0.025746 & 2.050510 & -2.165634 \\
\hline 0 & -0.421695 & 0.015087 & 2.453813 \\
\hline $\mathrm{H}$ & 0.397209 & 0.086724 & 2.959645 \\
\hline $\mathrm{H}$ & -1.061182 & -0.331059 & 3.088648 \\
\hline
\end{tabular}




$\begin{array}{lrrr}\mathrm{C} & 4.011758 & -0.718751 & 0.302223 \\ \mathrm{H} & 4.271281 & -1.764277 & 0.116202 \\ \mathrm{H} & 3.996249 & -0.581994 & 1.387947 \\ \mathrm{C} & 5.110385 & 0.145021 & -0.298763 \\ \mathrm{H} & 4.937137 & 1.208129 & -0.101295 \\ \mathrm{H} & 5.145251 & 0.001777 & -1.385241 \\ \mathrm{O} & 6.344211 & -0.267989 & 0.299098 \\ \mathrm{H} & 7.048441 & 0.286544 & -0.056260\end{array}$

$\mathrm{E}(\mathrm{UTPSSh})=-2564.1302239$ Hartree

Zero-point correction $=0.500626$

Thermal correction to Energy $=0.525600$

Thermal correction to Enthalpy $=0.526544$

Thermal correction to Gibbs Free Energy $=0.447259$

Sum of electronic and zero-point Energies $=-2563.629598$

Sum of electronic and thermal Energies $=-2563.604624$

Sum of electronic and thermal Enthalpies $=-2563.603680$

Sum of electronic and thermal Free Energies $=-2563.682965$

Table S12. Cartesian coordinates $(\AA)$ of $\left[\mathrm{Cu}(\mathbf{T M C})\left(\mathrm{H}_{2} \mathrm{O}\right)\right]^{2+}$ (trans-I) optimized at the uTPSSh/Def2-TZVPP level (scrf=pcm).

54

(uTMC) $\mathrm{H}_{2} \mathrm{O}$

\begin{tabular}{l} 
N \\
N \\
N \\
N \\
C \\
H \\
H \\
C \\
H \\
H \\
C \\
H \\
H \\
C \\
H \\
H \\
C \\
H \\
H \\
C \\
H \\
H \\
C \\
H \\
C \\
H \\
H \\
C \\
H \\
H \\
C \\
H \\
H \\
H \\
Cu \\
C \\
\hline
\end{tabular}

$$
\begin{array}{rr}
1.758343 & -1.204909 \\
1.016130 & 1.755931 \\
-1.765654 & 1.189225 \\
-1.021968 & -1.778129 \\
2.577460 & -0.924439 \\
1.974066 & -1.173935 \\
3.433788 & -1.607796 \\
3.073521 & 0.508176 \\
3.754110 & 0.527585 \\
3.678419 & 0.799218 \\
1.986059 & 1.528738 \\
2.443104 & 2.493428 \\
1.406454 & 1.204199 \\
-0.059406 & 2.652900 \\
0.313927 & 3.678913 \\
-0.312623 & 2.322033 \\
-1.262486 & 2.590219 \\
-1.010555 & 2.925393 \\
-2.058372 & 3.241311 \\
-2.589519 & 0.879053 \\
-1.989769 & 1.101676 \\
-3.444188 & 1.564727 \\
-3.087884 & -0.555756 \\
-3.689446 & -0.820578 \\
-2.000998 & -1.583725 \\
-2.458040 & -2.556177 \\
-1.427194 & -1.285510 \\
0.045085 & -2.692871 \\
0.287515 & -2.392094 \\
-0.333201 & -3.718769 \\
1.259210 & -2.610168 \\
1.021895 & -2.927426 \\
2.051953 & -3.265866 \\
-3.771133 & -0.601508 \\
-0.000959 & -0.004851 \\
-1.694236 & -2.414725
\end{array}
$$

0.184937 $-0.337721$

0.163885

$-0.276827$

$-1.034186$

$-1.907825$

$-1.017527$

$-1.158585$

$-2.013293$

$-0.298540$

$-1.450493$

$-1.698479$

$-2.316350$

$-0.850168$

$-0.922457$

$-1.856692$

0.056450

1.061562

$-0.317557$

$-1.044111$

$-1.927547$

$-1.042975$

$-1.123577$

$-0.252874$

$-1.388677$

$-1.604260$

$-2.267637$

$-0.776609$

$-1.795152$

$-0.816071$

0.112500

1.126930

$-0.259862$

$-1.975151$

0.194546

0.884154 


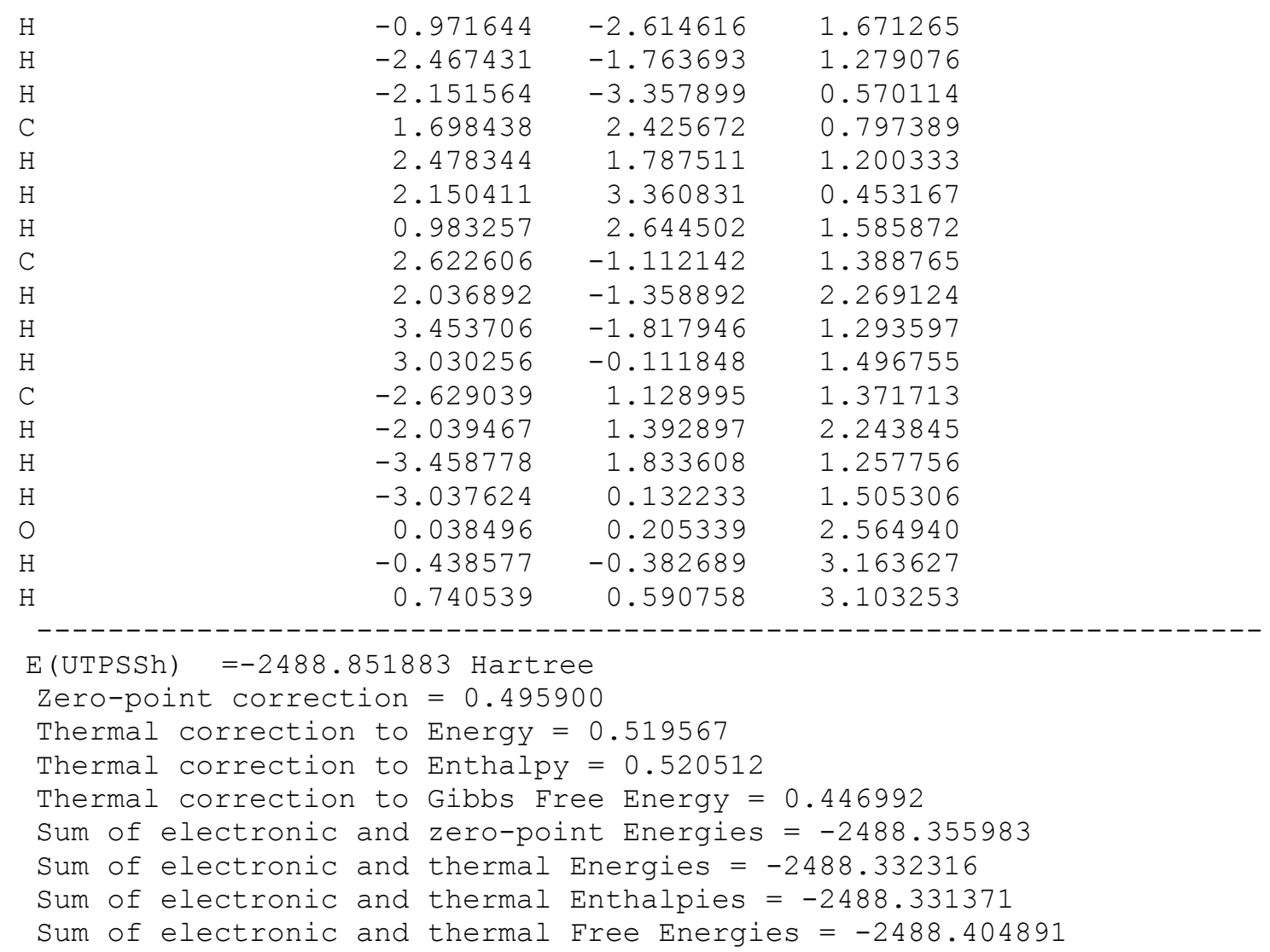

Table S13. Cartesian coordinates $(\AA)$ of $\left[\mathrm{Cu}(\mathbf{C B} \text {-cyclam })\left(\mathrm{H}_{2} \mathrm{O}\right)\right]^{2+}$ optimized at the uTPSSh/Def2-TZVPP level (scrf=pcm).

46

\section{$\mathrm{CuCBcyclam}_{2} \mathrm{O}$}

$\mathrm{C}$
$\mathrm{H}$
$\mathrm{C}$
$\mathrm{H}$
$\mathrm{C}$
$\mathrm{H}$
$\mathrm{H}$
$\mathrm{C}$
$\mathrm{H}$
$\mathrm{H}$
$\mathrm{C}$
$\mathrm{H}$
$\mathrm{H}$
$\mathrm{C}$
$\mathrm{H}$
$\mathrm{H}$
$\mathrm{C}$
$\mathrm{H}$
$\mathrm{C}$

$$
\begin{array}{r}
-2.967644 \\
-3.910963 \\
-2.957591 \\
-2.873779 \\
-2.804763 \\
-1.784370 \\
-1.911690 \\
-1.902819 \\
0.528274 \\
0.102487 \\
0.582109 \\
1.921804 \\
2.501168 \\
2.460518 \\
2.969303 \\
3.910212 \\
2.981031 \\
2.853945 \\
3.802198 \\
1.762447 \\
1.892771 \\
1.876063 \\
-0.546406 \\
-0.109026 \\
-0.615275 \\
-1.933632
\end{array}
$$

$$
\begin{array}{r}
-0.137893 \\
0.413653 \\
-0.845490 \\
-0.886518 \\
-0.195669 \\
-1.951546 \\
-2.540177 \\
-2.629783 \\
-2.554669 \\
-3.032585 \\
-3.303779 \\
-2.033650 \\
-2.810326 \\
-1.774031 \\
0.135129 \\
-0.421882 \\
0.843715 \\
0.882615 \\
1.404471 \\
1.947014 \\
2.625005 \\
2.536100 \\
2.549791 \\
3.035591 \\
3.293174 \\
2.028158
\end{array}
$$

$$
\begin{array}{r}
-0.606422 \\
-0.647845 \\
-1.436934 \\
0.719612 \\
1.562605 \\
0.790018 \\
1.705780 \\
-0.056648 \\
0.366294 \\
-0.516343 \\
1.164063 \\
0.052645 \\
-0.448771 \\
0.961500 \\
-0.552594 \\
-0.576755 \\
-1.382255 \\
0.772543 \\
0.923194 \\
0.825968 \\
-0.019200 \\
1.743294 \\
0.376702 \\
-0.495946 \\
1.178672 \\
0.038384
\end{array}
$$




\begin{tabular}{|c|c|c|c|}
\hline $\mathrm{H}$ & -0.635820 & -3.290442 & -1.308776 \\
\hline C & -1.959563 & -2.009906 & -0.189273 \\
\hline $\mathrm{H}$ & -2.497973 & -1.739839 & -1.095813 \\
\hline $\mathrm{H}$ & -2.542578 & -2.789921 & 0.303887 \\
\hline C & -0.073701 & -0.768580 & -2.141288 \\
\hline $\mathrm{H}$ & -1.114173 & -1.028898 & -2.317829 \\
\hline $\mathrm{H}$ & 0.493635 & -1.165582 & -2.989081 \\
\hline C & 0.073525 & 0.763151 & -2.143206 \\
\hline $\mathrm{H}$ & 1.113951 & 1.023174 & -2.320476 \\
\hline $\mathrm{H}$ & -0.493868 & 1.158018 & -2.991951 \\
\hline $\mathrm{N}$ & -1.875712 & -0.803234 & 0.676674 \\
\hline $\mathrm{N}$ & 0.356743 & -1.436999 & -0.884349 \\
\hline $\mathrm{N}$ & -0.357038 & 1.434640 & -0.887825 \\
\hline $\mathrm{N}$ & 1.875942 & 0.805000 & 0.674276 \\
\hline $\mathrm{Cl}$ & 0.000418 & 0.003533 & 2.998579 \\
\hline $\mathrm{H}$ & 2.782894 & -0.229773 & -1.732286 \\
\hline $\mathrm{Cu}$ & 0.000009 & 0.000995 & 0.682361 \\
\hline $\mathrm{H}$ & -3.791882 & 1.456708 & -1.037019 \\
\hline $\mathrm{H}$ & -1.925187 & -1.089333 & 1.652333 \\
\hline $\mathrm{H}$ & 1.925651 & 1.093355 & 1.649242 \\
\hline \multicolumn{4}{|c|}{$\mathrm{E}(\mathrm{UTPSS})=-2792.9550011$ Hartree } \\
\hline \multicolumn{4}{|c|}{ Zero-point correction $=0.396666$} \\
\hline \multicolumn{4}{|c|}{ Thermal correction to Energy $=0.414432$} \\
\hline \multicolumn{4}{|c|}{ Thermal correction to Enthalpy $=0.415376$} \\
\hline \multicolumn{4}{|c|}{ Thermal correction to Gibbs Free Energy $=0.352012$} \\
\hline \multicolumn{4}{|c|}{ Sum of electronic and zero-point Energies $=-2792.558335$} \\
\hline \multicolumn{4}{|c|}{ Sum of electronic and thermal Energies $=-2792.540569$} \\
\hline \multirow{2}{*}{\multicolumn{4}{|c|}{$\begin{array}{l}\text { Sum of electronic and thermal Enthalpies }=-2792.539625 \\
\text { Sum of electronic and thermal Free Energies }=-2792.602989\end{array}$}} \\
\hline & and thermal & Free Energi & $e s=-2792.602989$ \\
\hline
\end{tabular}

Table S15. Cartesian coordinates $(\AA)$ of $[\mathrm{Cu}(\mathbf{C B}-\mathbf{c y c l a m}-\mathbf{E})(\mathrm{Cl})]^{+}$optimized the uTPSSh/Def2-TZVPP level (scrf=pcm).

51

\section{CuCBcyclam-ECl}

$\mathrm{C}$
$\mathrm{H}$
$\mathrm{H}$
$\mathrm{C}$
$\mathrm{H}$
$\mathrm{C}$
$\mathrm{H}$
$\mathrm{H}$
$\mathrm{C}$
$\mathrm{H}$
$\mathrm{H}$
$\mathrm{C}$
$\mathrm{H}$
$\mathrm{H}$
$\mathrm{C}$
$\mathrm{H}$
$\mathrm{H}$
$\mathrm{C}$
$\mathrm{C}$
$\mathrm{H}$
$\mathrm{H}$
$\mathrm{C}$
$\mathrm{H}$

-2.064928
-2.855723
-1.993041
-2.431352
-2.356432
-1.552089
-1.970703
-1.597659
0.636857
0.345470
0.357756
2.139380
2.656121
2.490432
3.673660
4.492552
3.984578
3.395875
2.530720
2.967724
2.550250
0.534596
1.235406
0.427576

0.968436

1.702401

0.820227

$-0.361150$

$-0.265352$

$-1.527772$

$-2.462899$

$-1.579994$

$-2.427918$

$-2.227391$

$-3.461769$

$-2.248977$

$-2.787708$

$-2.664959$

$-0.470590$

$-1.153620$

0.537320

$-0.534036$

0.594265

1.547921

0.556491

1.950620

2. 675609

2. 161812
-0.719996
-0.540010
-1.799362
-0.049920
1.037542
-0.514631
-0.123065
-1.604762
-1.000487
-2.031956
-0.766266
-0.845361
-1.641656
0.096951
-0.029943
-0.275946
-0.309981
1.470382
2.026457
1.724405
3.122909
1.796067
1.380728
2.866990 


\begin{tabular}{|c|c|c|c|}
\hline $\mathrm{C}$ & 3.526203 & -0.291390 & -1.273085 \\
\hline $\mathrm{C}$ & 2.653036 & -1.541945 & -1.253164 \\
\hline $\mathrm{H}$ & 3.048943 & -2.235230 & -0.509064 \\
\hline $\mathrm{H}$ & 2.706845 & -2.037669 & -2.229335 \\
\hline $\mathrm{C}$ & 0.602298 & -2.604423 & -0.503088 \\
\hline $\mathrm{H}$ & 1.264293 & -3.056358 & 0.235965 \\
\hline $\mathrm{H}$ & 0.527448 & -3.290326 & -1.354200 \\
\hline $\mathrm{C}$ & -0.774321 & -2.380417 & 0.102754 \\
\hline $\mathrm{H}$ & -1.513598 & -2.161566 & -0.664605 \\
\hline $\mathrm{H}$ & -1.104287 & -3.286780 & 0.612367 \\
\hline $\mathrm{C}$ & 0.451870 & -0.650208 & -1.992040 \\
\hline $\mathrm{H}$ & -0.527719 & -1.118855 & -2.036041 \\
\hline $\mathrm{H}$ & 0.930040 & -0.840822 & -2.956999 \\
\hline $\mathrm{C}$ & 0.281085 & 0.873969 & -1.842751 \\
\hline $\mathrm{H}$ & 1.188831 & 1.372479 & -2.171480 \\
\hline $\mathrm{H}$ & -0.511614 & 1.191427 & -2.526067 \\
\hline $\mathrm{N}$ & -0.748058 & -1.236412 & 1.059598 \\
\hline $\mathrm{N}$ & 1.227179 & -1.313262 & -0.906124 \\
\hline $\mathrm{N}$ & -0.025467 & 1.347867 & -0.462706 \\
\hline $\mathrm{N}$ & 2.542437 & 1.069687 & 0.620759 \\
\hline $\mathrm{H}$ & -0.564354 & -1.601367 & 1.992281 \\
\hline $\mathrm{H}$ & 2.750809 & 1.319988 & 1.584353 \\
\hline $\mathrm{Cu}$ & 0.892247 & -0.087133 & 0.799772 \\
\hline $\mathrm{H}$ & 4.509329 & -0.605383 & -1.632306 \\
\hline C & -3.826933 & 0.809728 & -0.050886 \\
\hline $\mathrm{H}$ & -4.049077 & 1.195884 & 0.949006 \\
\hline $\mathrm{H}$ & -3.969616 & 1.637464 & -0.750427 \\
\hline C & -4.831414 & -0.276419 & -0.403749 \\
\hline $\mathrm{H}$ & -4.753921 & -1.129856 & 0.278408 \\
\hline $\mathrm{H}$ & -4.647499 & -0.638096 & -1.422293 \\
\hline O & -6.135362 & 0.307945 & -0.309573 \\
\hline $\mathrm{H}$ & -6.784528 & -0.375003 & -0.514151 \\
\hline $\mathrm{H}$ & 3.166895 & 0.427417 & -2.012563 \\
\hline O & 1.200465 & -0.354154 & 2.902513 \\
\hline $\mathrm{H}$ & 2.009456 & -0.777736 & 3.218755 \\
\hline $\mathrm{H}$ & 1.032109 & 0.386130 & 3.500544 \\
\hline \multicolumn{4}{|c|}{$E($ UTPSSh $)=-2562.9022632$ Hartree } \\
\hline \multicolumn{4}{|c|}{ Zero-point correction $=0.482717$} \\
\hline \multicolumn{4}{|c|}{ Thermal correction to Energy $=0.505250$} \\
\hline \multicolumn{4}{|c|}{ Thermal correction to Enthalpy $=0.506194$} \\
\hline \multicolumn{4}{|c|}{ Thermal correction to Gibbs Free Energy $=0.433421$} \\
\hline \multicolumn{4}{|c|}{ Sum of electronic and zero-point Energies $=-2562.419546$} \\
\hline \\
\hline \multicolumn{4}{|c|}{$\begin{array}{l}\text { Sum of electronic and thermal Energies }=-2562.397013 \\
\text { Sum of electronic and thermal Enthalpies }=-2562.396069\end{array}$} \\
\hline Sum of electronic a & and therma. & Free Energi & $e s=-2562.468843$ \\
\hline
\end{tabular}

Table S17. Cartesian coordinates $(\AA)$ of $[\mathrm{Cu}(\mathbf{C B}-\mathrm{DMC})(\mathrm{Cl})]^{+}$optimized the uTPSSh/Def2-TZVPP level (scrf=pcm).

50

\section{$\mathrm{CuCBDMCCl}$}

$\mathrm{C}$
$\mathrm{H}$
$\mathrm{H}$
$\mathrm{C}$
$\mathrm{H}$
$\mathrm{C}$
$\mathrm{H}$
$\mathrm{H}$

$$
\begin{aligned}
& -2.896760 \\
& -3.946041 \\
& -2.819560 \\
& -2.471772 \\
& -2.464695 \\
& -1.179365 \\
& -1.136773 \\
& -1.201953
\end{aligned}
$$

$$
\begin{array}{r}
-0.538744 \\
-0.239671 \\
-1.598643 \\
-0.310826 \\
0.751081 \\
-0.987617 \\
-0.966661 \\
-2.033356
\end{array}
$$

0.331849
0.219891
0.089895
1.785002
2.043104
2.241424
3.338834
1.929591 


\begin{tabular}{|c|c|c|c|}
\hline C & 1.189387 & -1.353792 & 1.856817 \\
\hline $\mathrm{H}$ & 0.842825 & -2.322733 & 1.495310 \\
\hline $\mathrm{H}$ & 1.471619 & -1.462705 & 2.912326 \\
\hline C & 2.412820 & -0.916122 & 1.065938 \\
\hline $\mathrm{H}$ & 3.182388 & -1.688582 & 1.144242 \\
\hline $\mathrm{H}$ & 2.839432 & -0.011404 & 1.493663 \\
\hline $\mathrm{C}$ & 2.963781 & 0.459492 & -0.881505 \\
\hline $\mathrm{H}$ & 4.010163 & 0.199865 & -0.680495 \\
\hline $\mathrm{H}$ & 2.838840 & 0.484675 & -1.966051 \\
\hline $\mathrm{C}$ & 2.672309 & 1.837493 & -0.309670 \\
\hline $\mathrm{H}$ & 3.469242 & 2.495255 & -0.666005 \\
\hline C & 1.362490 & 2.441572 & -0.778829 \\
\hline $\mathrm{H}$ & 1.296053 & 2.354551 & -1.864859 \\
\hline $\mathrm{H}$ & 1.335025 & 3.508542 & -0.529745 \\
\hline C & -1.021829 & 2.373988 & -0.964595 \\
\hline $\mathrm{H}$ & -0.811115 & 2.247494 & -2.026921 \\
\hline $\mathrm{H}$ & -1.111819 & 3.447699 & -0.766474 \\
\hline C & -2.297601 & 1.657454 & -0.591569 \\
\hline $\mathrm{H}$ & -2.599057 & 1.893291 & 0.425665 \\
\hline $\mathrm{H}$ & -3.111795 & 1.984627 & -1.242954 \\
\hline C & -0.010599 & 2.087719 & 1.247967 \\
\hline $\mathrm{H}$ & -1.048951 & 2.352067 & 1.423879 \\
\hline $\mathrm{H}$ & 0.579728 & 2.972266 & 1.500907 \\
\hline C & 0.382115 & 0.939970 & 2.197211 \\
\hline $\mathrm{H}$ & 1.449017 & 0.986789 & 2.401603 \\
\hline $\mathrm{H}$ & -0.118999 & 1.114003 & 3.155014 \\
\hline $\mathrm{N}$ & -2.109590 & 0.185033 & -0.712390 \\
\hline $\mathrm{N}$ & 0.139344 & 1.805762 & -0.218539 \\
\hline $\mathrm{N}$ & 0.063417 & -0.409472 & 1.685817 \\
\hline $\mathrm{N}$ & 2.114797 & -0.660141 & -0.378685 \\
\hline $\mathrm{Cl}$ & -0.505257 & -2.468206 & -1.054683 \\
\hline $\mathrm{H}$ & 2.748531 & 1.855218 & 0.779694 \\
\hline C & 2.517183 & -1.856670 & -1.164507 \\
\hline $\mathrm{H}$ & 2.203956 & -1.733728 & -2.199111 \\
\hline $\mathrm{H}$ & 3.606855 & -1.962548 & -1.120014 \\
\hline $\mathrm{H}$ & 2.041363 & -2.741492 & -0.756318 \\
\hline C & -2.596336 & -0.233303 & -2.052044 \\
\hline $\mathrm{H}$ & -2.430371 & -1.298685 & -2.176366 \\
\hline $\mathrm{H}$ & -3.663043 & -0.001652 & -2.141169 \\
\hline $\mathrm{H}$ & -2.047581 & 0.310018 & -2.820697 \\
\hline $\mathrm{Cu}$ & -0.039748 & -0.284371 & -0.496376 \\
\hline $\mathrm{H}$ & -3.271965 & -0.741248 & 2.393718 \\
\hline \\
\hline \multicolumn{4}{|c|}{ Zero-point correction $=0.451812$} \\
\hline \multicolumn{4}{|c|}{ Thermal correction to Energy $=0.472113$} \\
\hline \multicolumn{4}{|c|}{ Thermal correction to Enthalpy $=0.473057$} \\
\hline \multicolumn{4}{|c|}{ Thermal correction to Gibbs Free Energy $=0.406068$} \\
\hline \multicolumn{4}{|c|}{ Sum of electronic and zero-point Energies $=-2871.130495$} \\
\hline Sum of electronic a & and thermal & Energies $=$ & -2871.110194 \\
\hline Sum of electronic a & and thermal & Enthalpies & $=-2871.109250$ \\
\hline Sum of electronic a & and thermal & Free Energi & $e s=-2871.176240$ \\
\hline
\end{tabular}

Table S18. Cartesian coordinates $(\AA)$ of $\left[\mathrm{Cu}(\mathbf{C B}-\mathrm{DMC})\left(\mathrm{H}_{2} \mathrm{O}\right)\right]^{2+}$ optimized at the uTPSSh/Def2-TZVPP level ( $\mathrm{scrf}=\mathrm{pcm})$.

52

\section{$\mathrm{CuCBDMCH}_{2} \mathrm{O}$}

$\begin{array}{llll}\mathrm{C} & -2.861764 & -0.796085 & -0.001294 \\ \mathrm{H} & -3.917110 & -0.515009 & -0.089151 \\ \mathrm{H} & -2.733543 & -1.743619 & -0.525346\end{array}$




\begin{tabular}{|c|c|c|c|}
\hline $\mathrm{C}$ & -2.519070 & -0.965449 & 1.480288 \\
\hline $\mathrm{H}$ & -2.572953 & -0.015688 & 2.016759 \\
\hline C & -1.219606 & -1.697664 & 1.809089 \\
\hline $\mathrm{H}$ & -1.199752 & -1.924078 & 2.882052 \\
\hline $\mathrm{H}$ & -1.204090 & -2.651709 & 1.278676 \\
\hline C & 1.174209 & -1.896432 & 1.380319 \\
\hline $\mathrm{H}$ & 0.869285 & -2.767469 & 0.798647 \\
\hline $\mathrm{H}$ & 1.451842 & -2.253901 & 2.379010 \\
\hline C & 2.383489 & -1.230668 & 0.743060 \\
\hline $\mathrm{H}$ & 3.166806 & -1.975818 & 0.587810 \\
\hline $\mathrm{H}$ & 2.798587 & -0.477656 & 1.407914 \\
\hline C & 2.904068 & 0.653445 & -0.737718 \\
\hline $\mathrm{H}$ & 3.949597 & 0.348647 & -0.621031 \\
\hline $\mathrm{H}$ & 2.772061 & 0.981878 & -1.770235 \\
\hline C & 2.608792 & 1.809422 & 0.205496 \\
\hline $\mathrm{H}$ & 3.405858 & 2.541122 & 0.052520 \\
\hline C & 1.299405 & 2.525208 & -0.071493 \\
\hline $\mathrm{H}$ & 1.244261 & 2.777159 & -1.131621 \\
\hline $\mathrm{H}$ & 1.259888 & 3.461019 & 0.496227 \\
\hline C & -1.091907 & 2.449202 & -0.344180 \\
\hline $\mathrm{H}$ & -0.849657 & 2.648370 & -1.388056 \\
\hline $\mathrm{H}$ & -1.244977 & 3.411103 & 0.155430 \\
\hline C & -2.334807 & 1.596712 & -0.239467 \\
\hline $\mathrm{H}$ & -2.676835 & 1.522414 & 0.788994 \\
\hline $\mathrm{H}$ & -3.147833 & 2.051244 & -0.809357 \\
\hline C & -0.115633 & 1.562723 & 1.725582 \\
\hline $\mathrm{H}$ & -1.165946 & 1.735225 & 1.939310 \\
\hline $\mathrm{H}$ & 0.439776 & 2.343567 & 2.250348 \\
\hline C & 0.301135 & 0.197710 & 2.305454 \\
\hline $\mathrm{H}$ & 1.365667 & 0.207187 & 2.522993 \\
\hline $\mathrm{H}$ & -0.205861 & 0.078942 & 3.267218 \\
\hline $\mathrm{N}$ & -2.063909 & 0.221732 & -0.756815 \\
\hline N & 0.078486 & 1.737534 & 0.249117 \\
\hline $\mathrm{N}$ & 0.016220 & -0.970379 & 1.433645 \\
\hline $\mathrm{N}$ & 2.060726 & -0.571850 & -0.566373 \\
\hline $\mathrm{H}$ & 2.685768 & 1.515756 & 1.254383 \\
\hline C & 2.448772 & -1.494585 & -1.669805 \\
\hline $\mathrm{H}$ & 2.124124 & -1.083748 & -2.624193 \\
\hline $\mathrm{H}$ & 3.537375 & -1.601330 & -1.680481 \\
\hline $\mathrm{H}$ & 2.004864 & -2.473119 & -1.514112 \\
\hline C & -2.472503 & 0.178875 & -2.188523 \\
\hline $\mathrm{H}$ & -2.319628 & -0.819920 & -2.582476 \\
\hline $\mathrm{H}$ & -3.531077 & 0.442111 & -2.269447 \\
\hline $\mathrm{H}$ & -1.881576 & 0.894558 & -2.758310 \\
\hline $\mathrm{Cu}$ & -0.022512 & -0.183769 & -0.580923 \\
\hline $\mathrm{H}$ & -3.325282 & -1.571656 & 1.901708 \\
\hline O & -0.444403 & -1.908772 & -1.766701 \\
\hline $\mathrm{H}$ & -0.158812 & -1.941847 & -2.688966 \\
\hline $\mathrm{H}$ & -0.400891 & -2.817998 & -1.444711 \\
\hline
\end{tabular}

$E($ UTPSSh $)=-2487.6251241$ Hartree

Zero-point correction $=0.477495$

Thermal correction to Energy $=0.498309$

Thermal correction to Enthalpy $=0.499253$

Thermal correction to Gibbs Free Energy $=0.432219$

Sum of electronic and zero-point Energies $=-2487.147629$

Sum of electronic and thermal Energies $=-2487.126816$

Sum of electronic and thermal Enthalpies $=-2487.125871$

Sum of electronic and thermal Free Energies = -2487.192905 
Table S19. Cartesian coordinates $(\AA)$ of $[\mathrm{Cu}(\mathbf{C B}-\mathrm{DMC}-\mathbf{E})(\mathrm{Cl})]^{+}$optimized at the uTPSSh/Def2-TZVPP level (scrf=pcm).

57

\section{CUCBDMCECl}

C

$\mathrm{H}$

$\mathrm{H}$

C

$\mathrm{H}$

$\mathrm{H}$

$\mathrm{H}$

C

$\mathrm{H}$

C

H

$\mathrm{H}$

$\mathrm{H}$

C

$\mathrm{H}$

$\mathrm{H}$

$\mathrm{H}$

C

$\mathrm{H}$

C

$\mathrm{H}$

$\mathrm{H}$

C

$\mathrm{H}$

$\mathrm{H}$

$\mathrm{H}$

$\mathrm{H}$

$\mathrm{N}$

N

N

$\mathrm{N}$

C

$\mathrm{H}$

C

$\mathrm{H}$

$\mathrm{H}$

C

$\mathrm{H}$

H

$\mathrm{H}$

C

$\mathrm{H}$

$\mathrm{H}$

$\mathrm{H}$

C

$\mathrm{H}$

$\mathrm{H}$

$$
\begin{aligned}
& -2.158757 \\
& -3.050160 \\
& -2.033071 \\
& -2.379497 \\
& -2.332339 \\
& -1.391615 \\
& -1.773389 \\
& -1.355282 \\
& 0.906674 \\
& 0.681745 \\
& 0.730019 \\
& 2.368090 \\
& \text { 2. } 995422 \\
& \text { 2. } 633868 \\
& \text { 3. } 744240 \\
& \text { 4. } 598810 \\
& \text { 4. } 075341 \\
& \text { 3. } 329208 \\
& \text { 4. } 239972 \\
& \text { 2. } 359686 \\
& \text { 2. } 728354 \\
& \text { 2. } 300019 \\
& 0.243177 \\
& 0.845595 \\
& 0.148369 \\
& -1.117913 \\
& -1.778677 \\
& -1.590725 \\
& 0.278671 \\
& -0.718706 \\
& 0.787531 \\
& 0.167269 \\
& 1.054981 \\
& -0.671703 \\
& -0.983209 \\
& 0.979085 \\
& -0.000239 \\
& \text { 2. } 697559 \\
& 0.474100 \\
& \text { 2. } 956500 \\
& -3.807768 \\
& -4.008224 \\
& -3.877382 \\
& -4.891673 \\
& -4.872030 \\
& -4.739521 \\
& -6.151712 \\
& -6.846930 \\
& \text { 3. } 312065 \\
& \text { 3. } 450818 \\
& \text { 4. } 282549 \\
& \text { 2. } 661149 \\
& -0.921852 \\
& -0.785508 \\
& -1.848619 \\
& -0.080101
\end{aligned}
$$

0.990100

1.471645

1.349887

$-0.532598$

$-0.947579$

$-1.300118$

$-2.319773$

$-0.817279$

$-1.781766$

$-1.139793$

$-2.823434$

$-1.626314$

$-1.857560$

$-2.343220$

$-0.334773$

$-0.880516$

0.687777

$-0.983917$

$-1.091480$

$-0.158024$

0.867099

$-0.559931$

0.931337

1.840270

0.597086

1.198842

0.345338

2.039434

$-1.410373$

$-1.226575$

$-2.031653$

$-2.203711$

$-2.818434$

$-2.899605$

1.499404

$-0.085930$

$-1.367110$

$-0.252888$

1. 697668

$-2.000374$

$-0.794320$

$-0.150754$

$-1.827056$

$-0.582614$

0.439337

$-1.272083$

$-0.838707$

$-0.700812$

0.518190

1.552399

0.074098

0.494133

2. 976370

3. 219814

3.421834

3. 363418 


$\mathrm{Cu} 0.801825 \quad 0.172658 \quad 0.613843$
E(UTPSSh) $=-3025.4883932$ Hartree
Zero-point correction 0.512523
Thermal correction to Energy $=0.537070$
Thermal correction to Enthalpy $=0.538014$
Thermal correction to Gibs Free Energy $=0.460798$
Sum of electronic and zero-point Energies $=-3024.975870$
Sum of electronic and thermal Energies $=-3024.951323$
Sum of electronic and thermal Enthalpies $=-3024.950379$
Sum of electronic and thermal Free Energies $=-3025.027595$

Table S20. Cartesian coordinates $(\AA)$ of $\left[\mathrm{Cu}(\mathbf{C B}-\mathrm{DMC}-\mathbf{E})\left(\mathrm{H}_{2} \mathrm{O}\right)\right]^{2+}$ optimized at the uTPSSh/Def2-TZVPP level (scrf=pcm).

\section{CUCBDMCEH2O}

$\mathrm{C}$
$\mathrm{H}$
$\mathrm{H}$
$\mathrm{C}$
$\mathrm{H}$
$\mathrm{C}$
$\mathrm{H}$
$\mathrm{H}$
$\mathrm{H}$
$\mathrm{C}$
$\mathrm{H}$
$\mathrm{H}$
$\mathrm{C}$
$\mathrm{H}$
$\mathrm{H}$
$\mathrm{C}$
$\mathrm{H}$
$\mathrm{H}$
$\mathrm{H}$
$\mathrm{C}$
$\mathrm{H}$
$\mathrm{C}$
$\mathrm{H}$
$\mathrm{H}$
$\mathrm{C}$
$\mathrm{H}$
$\mathrm{H}$
$\mathrm{H}$
$\mathrm{C}$
$\mathrm{H}$
$\mathrm{H}$
$\mathrm{C}$
$\mathrm{H}$
$\mathrm{H}$
$\mathrm{C}$
$\mathrm{H}$
$\mathrm{H}$
$\mathrm{H}$
$\mathrm{N}$
$\mathrm{N}$
$\mathrm{N}$
$\mathrm{N}$
$\mathrm{H}$
$\mathrm{C}$
$\mathrm{H}$
$\mathrm{H}$
$\mathrm{H}$
$\mathrm{C}$
$\mathrm{C}$

$$
\begin{array}{r}
2.118313 \\
3.000019 \\
1.985773 \\
2.382195 \\
2.343583 \\
1.421356 \\
1.785124 \\
1.444228 \\
-0.878858 \\
-0.617791 \\
-0.711651 \\
-2.343903 \\
-2.957817 \\
-2.650992 \\
-3.727617 \\
-4.582041 \\
-4.027396 \\
-3.379404 \\
-4.316410 \\
-2.422957 \\
-2.778217 \\
-2.398188 \\
-0.290640 \\
-0.891702 \\
-0.189937 \\
1.070141 \\
1.726888 \\
1.547933 \\
-0.331598 \\
0.662363 \\
-0.850649 \\
-0.215466 \\
-1.116910 \\
0.600833 \\
0.936883 \\
-1.028166 \\
0.006759 \\
-2.640229 \\
-3.031278 \\
3.817240 \\
4.007233 \\
3.907980 \\
4.891191 \\
-
\end{array}
$$

$$
\begin{array}{r}
1.015112 \\
1.248210 \\
1.839738 \\
-0.282523 \\
-1.145833 \\
-0.528089 \\
-1.388081 \\
0.337404 \\
-0.553117 \\
0.411039 \\
-1.317548 \\
-0.572958 \\
-0.289537 \\
-1.576551 \\
-0.228696 \\
-0.431041 \\
0.555727 \\
-1.490601 \\
-1.855553 \\
-1.274511 \\
-0.443885 \\
-2.168532 \\
-0.495902 \\
0.283762 \\
-1.318404 \\
0.038130 \\
-0.760042 \\
0.496684 \\
-2.123547 \\
-2.182725 \\
-3.039320 \\
-2.103372 \\
-2.526212 \\
-2.775289 \\
1.046004 \\
-0.950832 \\
-0.761801 \\
0.349367 \\
-2.291775 \\
-0.215611 \\
0.780797 \\
-0.923345 \\
-0.549157
\end{array}
$$




$\begin{array}{lrrr}\mathrm{H} & 4.839094 & 0.824758 & 0.121498 \\ \mathrm{H} & 4.756376 & 0.319301 & -1.575994 \\ \mathrm{O} & 6.158407 & -0.691993 & -0.410972 \\ \mathrm{H} & 6.847946 & -0.052382 & -0.623723 \\ \mathrm{C} & -3.186502 & -1.468325 & 1.619870 \\ \mathrm{H} & -3.337816 & -0.665013 & 2.337745 \\ \mathrm{H} & -4.149096 & -1.945470 & 1.414588 \\ \mathrm{H} & -2.509569 & -2.212645 & 2.028478 \\ \mathrm{C} & 0.880173 & 1.837289 & 2.395363 \\ \mathrm{H} & 0.834124 & 1.068978 & 3.158474 \\ \mathrm{H} & 1.776845 & 2.445530 & 2.543389 \\ \mathrm{H} & 0.000356 & 2.475483 & 2.463556 \\ \mathrm{Cu} & -0.817976 & 0.130758 & 0.657990 \\ \mathrm{O} & -0.589528 & -0.638287 & 2.622935 \\ \mathrm{H} & -1.182741 & -0.359202 & 3.332066 \\ \mathrm{H} & -0.344916 & -1.550866 & 2.821309 \\ ------------------------------------------------------\end{array}$

$\mathrm{E}(\mathrm{UTPSSh})=-2641.531208$ Hartree

Zero-point correction $=0.537408$

Thermal correction to Energy $=0.562912$

Thermal correction to Enthalpy $=0.563856$

Thermal correction to Gibbs Free Energy $=0.485304$

Sum of electronic and zero-point Energies $=-2640.993800$

Sum of electronic and thermal Energies $=-2640.968296$

Sum of electronic and thermal Enthalpies $=-2640.967352$

Sum of electronic and thermal Free Energies $=-2641.045904$

Table S21. Cartesian coordinates $(\AA)$ of $[\mathrm{Zn}(\mathbf{D M C}) \mathrm{Cl}]^{+}$(trans-I) optimized at the rTPSSh/Def2-TZVPP level ( $\mathrm{scrf}=\mathrm{pcm})$.

$$
46
$$

\section{$\mathrm{ZnDMCCl}$}

$\mathrm{N}$

$\mathrm{N}$

$\mathrm{N}$

$\mathrm{N}$

C

$\mathrm{H}$

$\mathrm{H}$

C

$\mathrm{H}$

$\mathrm{H}$

C

$\mathrm{H}$

$\mathrm{H}$

C

$\mathrm{H}$

$\mathrm{H}$

$\mathrm{H}$

$\mathrm{H}$

C

$\mathrm{H}$

$\mathrm{H}$

C

C

$\mathrm{H}$

$\mathrm{H}$

C

$$
\begin{array}{r}
-0.425752 \\
-2.034141 \\
0.425917 \\
2.033965 \\
-1.255082 \\
-0.702655 \\
-1.397066 \\
-2.610303 \\
-3.206959 \\
-3.144028 \\
-2.571645 \\
-3.584258 \\
-1.945049 \\
-1.878013 \\
-2.846471 \\
-1.514075 \\
-0.891664 \\
-1.231974 \\
-0.809218 \\
1.254882 \\
0.702307 \\
1.396811 \\
2.610174 \\
3.144073 \\
2.571603 \\
3.584297 \\
1.945201 \\
1.878177
\end{array}
$$

$$
\begin{array}{r}
0.067998 \\
-0.287687 \\
0.066688 \\
-0.287596 \\
-1.104951 \\
-2.006381 \\
-1.120590 \\
-1.102178 \\
-1.897911 \\
-0.172505 \\
-1.388362 \\
-1.630538 \\
-2.264814 \\
-0.783229 \\
-0.802687 \\
-1.810244 \\
0.072395 \\
1.106722 \\
-0.303942 \\
-1.106760 \\
-2.007860 \\
-1.123375 \\
-1.103755 \\
-0.174359 \\
-1.388802 \\
-1.630519 \\
-2.265248 \\
-0.782156
\end{array}
$$




\begin{tabular}{|c|c|c|c|}
\hline $\mathrm{H}$ & 1.514466 & 1.930077 & -1.809291 \\
\hline $\mathrm{H}$ & 2.846716 & 2.492926 & -0.800947 \\
\hline $\mathrm{C}$ & 0.891802 & 2.762052 & 0.073821 \\
\hline $\mathrm{H}$ & 1.232204 & 2.813148 & 1.108122 \\
\hline $\mathrm{H}$ & 0.809127 & 3.786260 & -0.302293 \\
\hline $\mathrm{Cl}$ & 0.000750 & -0.002056 & 2.700417 \\
\hline $\mathrm{H}$ & 3.206583 & -2.209312 & -1.900004 \\
\hline $\mathrm{Zn}$ & -0.000294 & 0.000115 & 0.387386 \\
\hline $\mathrm{H}$ & 0.925746 & -2.365896 & 0.906870 \\
\hline $\mathrm{H}$ & -0.925346 & 2.365473 & 0.908534 \\
\hline C & 2.969262 & 0.580955 & 0.859936 \\
\hline $\mathrm{H}$ & 2.603664 & 1.248160 & 1.637000 \\
\hline $\mathrm{H}$ & 3.035339 & -0.418112 & 1.284053 \\
\hline $\mathrm{H}$ & 3.965626 & 0.904600 & 0.536921 \\
\hline C & -2.969673 & -0.581637 & 0.859673 \\
\hline $\mathrm{H}$ & -3.036478 & 0.417223 & 1.284152 \\
\hline $\mathrm{H}$ & -3.965767 & -0.905761 & 0.536322 \\
\hline $\mathrm{H}$ & -2.603812 & -1.248921 & 1.636546 \\
\hline \\
\hline \multirow{2}{*}{\multicolumn{4}{|c|}{$\begin{array}{l}\text { Zero-point correction }=0.415806 \\
\text { Thermal correction to Energy }=0.435940\end{array}$}} \\
\hline & & & \\
\hline \multicolumn{4}{|c|}{$\begin{array}{l}\text { Thermal correction to Energy }=0.435940 \\
\text { Thermal correction to Enthalpy }=0.436884\end{array}$} \\
\hline \multicolumn{4}{|c|}{ Thermal correction to Gibbs Free Energy $=0.369269$} \\
\hline \multicolumn{4}{|c|}{ Sum of electronic and zero-point Energies $=-2932.638742$} \\
\hline \multicolumn{4}{|c|}{ Sum of electronic and thermal Energies $=-2932.618608$} \\
\hline Sum of electronic a & and thermal & Enthalpies & $=-2932.617663$ \\
\hline Sum of electronic a & and thermal & Free Energi & $e s=-2932.685279$ \\
\hline
\end{tabular}

Table S22. Cartesian coordinates $(\AA)$ of $[\mathrm{Zn}(\mathbf{D M C}) \mathrm{Cl}]^{+}$(trans-III) optimized at the rTPSSh/Def2-TZVPP level $(\mathrm{scrf}=\mathrm{pcm})$.

46

\section{$\mathrm{ZnDMCCl}$}

$\mathrm{H}$

C

$\mathrm{H}$

$\mathrm{H}$

C

$\mathrm{H}$

$\mathrm{H}$

$\mathrm{N}$

C

$\mathrm{H}$

$\mathrm{N}$

$\mathrm{H}$

$\mathrm{H}$

C

$\mathrm{H}$

Zn

$\mathrm{H}$

C

$\mathrm{H}$

C

$\mathrm{H}$

$\mathrm{N}$

$\mathrm{H}$

C

$\mathrm{N}$

\begin{abstract}
2.590183
0.436003

0.406330

1.795001

1. 756746

$-2.228059$

$-0.961934$

$-0.714398$

$-1.907588$

$-1.600088$

1.885553

2. 828132

2. 997742

$-3.938663$

$-3.075401$

$-3.345178$

$-0.027589$

3. 290710

3. 112622

4.016896

$-2.892257$

$-2.458605$

$-2.009871$

1. 791048

1. 964512

0.690021
\end{abstract}

$$
\begin{array}{r}
2.894515 \\
2.913283 \\
3.220936 \\
1.969647 \\
2.218189 \\
3.393530 \\
2.050219 \\
2.001996 \\
2.383819 \\
2.413503 \\
0.948260 \\
0.054389 \\
0.128166 \\
1.877770 \\
1.416268 \\
1.325294 \\
-0.023656 \\
-1.231455 \\
-1.277904 \\
-1.720142 \\
0.041977 \\
0.167509 \\
-0.893968 \\
-2.316294 \\
-2.243189 \\
-1.797310
\end{array}
$$

$$
\begin{array}{r}
0.129554 \\
0.034673 \\
-1.010402 \\
1.417435 \\
0.355353 \\
-0.253840 \\
1.250249 \\
0.263608 \\
-0.531455 \\
-1.578882 \\
-0.395640 \\
1.231139 \\
0.155737 \\
-0.850586 \\
-0.364209 \\
0.691263 \\
0.161997 \\
-1.518069 \\
-0.440116 \\
-0.013791 \\
-1.010493 \\
-2.004785 \\
-0.259312 \\
0.926779 \\
-0.148371 \\
-0.763628
\end{array}
$$




\begin{tabular}{|c|c|c|c|}
\hline $\mathrm{H}$ & -3.207332 & 1.978306 & -1.035061 \\
\hline $\mathrm{H}$ & -2.172958 & 0.864802 & -1.926525 \\
\hline $\mathrm{N}$ & -0.172686 & -1.566224 & -1.067264 \\
\hline $\mathrm{N}$ & -2.241222 & 0.488325 & 0.140731 \\
\hline $\mathrm{C}$ & -1.364057 & -2.437531 & -0.911667 \\
\hline $\mathrm{H}$ & -2.585186 & -1.183056 & -2.168086 \\
\hline $\mathrm{H}$ & -1.357517 & -2.836548 & 0.104593 \\
\hline $\mathrm{C}$ & -3.107193 & -0.695771 & -0.108399 \\
\hline $\mathrm{H}$ & -1.288457 & -3.282485 & -1.604051 \\
\hline $\mathrm{H}$ & -3.170446 & -1.230824 & 0.841474 \\
\hline $\mathrm{C}$ & -2.652636 & -1.671290 & -1.190590 \\
\hline $\mathrm{H}$ & -4.120236 & -0.345672 & -0.350102 \\
\hline $\mathrm{H}$ & -3.455000 & -2.406925 & -1.288948 \\
\hline $\mathrm{Cl}$ & -0.026191 & -0.183447 & 2.680074 \\
\hline $\mathrm{C}$ & 2.775715 & -1.249623 & 1.261802 \\
\hline $\mathrm{H}$ & 3.817650 & -1.535642 & 1.070786 \\
\hline $\mathrm{H}$ & 2.720243 & -0.637836 & 2.158507 \\
\hline $\mathrm{H}$ & 2.182785 & -2.146454 & 1.426440 \\
\hline C & -2.776719 & 1.167945 & 1.340693 \\
\hline $\mathrm{H}$ & -3.818619 & 1.467988 & 1.171643 \\
\hline $\mathrm{H}$ & -2.721228 & 0.489886 & 2.188446 \\
\hline $\mathrm{H}$ & -2.184515 & 2.050627 & 1.572638 \\
\hline \multicolumn{4}{|c|}{$E($ RTPSSh $)=-2933.0493139$ Hartree } \\
\hline \\
\hline \multirow{2}{*}{\multicolumn{4}{|c|}{$\begin{array}{l}\text { Thermal correction to Energy }=0.435669 \\
\text { Thermal correction to Enthalpy }=0.436613\end{array}$}} \\
\hline & & & \\
\hline \multicolumn{4}{|c|}{ Thermal correction to Gibbs Free Energy $=0.369375$} \\
\hline \multicolumn{4}{|c|}{ Sum of electronic and zero-point Energies $=-2932.633773$} \\
\hline \multicolumn{4}{|l|}{ Sum of electronic a } \\
\hline \multirow{2}{*}{\multicolumn{4}{|c|}{$\begin{array}{l}\text { Sum of electronic and thermal Enthalpies }=-2932.612701 \\
\text { Sum of electronic and thermal Free Energies }=-2932.679939\end{array}$}} \\
\hline & and therma. & Free Energi & $e s=-2932.679939$ \\
\hline
\end{tabular}

Table S24. Cartesian coordinates $(\AA)$ of $[\mathrm{Zn}(\text { cyclam }) \mathrm{Cl}]^{+}$(trans-I) optimized at the rTPSSh/Def2-TZVPP level (scrf=pcm).

$\begin{array}{lrrr}40 & & \\ \text { ZncyclamCl } & -2.002292 & 0.785799 & 0.208141 \\ \mathrm{~N} & -0.564403 & -1.946446 & -0.282267 \\ \mathrm{~N} & 2.002411 & -0.785939 & 0.209014 \\ \mathrm{~N} & 0.564577 & 1.946433 & -0.282365 \\ \mathrm{~N} & -2.835286 & 0.239890 & -0.888618 \\ \mathrm{C} & -2.394973 & 0.557618 & -1.836085 \\ \mathrm{H} & -3.840564 & 0.671472 & -0.832568 \\ \mathrm{H} & -2.924312 & -1.283404 & -0.847202 \\ \mathrm{C} & -3.722546 & -1.582427 & -1.530973 \\ \mathrm{H} & -3.237238 & -1.616011 & 0.148510 \\ \mathrm{H} & -1.661148 & -2.022920 & -1.280252 \\ \mathrm{C} & -1.901410 & -3.073050 & -1.476937 \\ \mathrm{H} & -1.279918 & -1.594465 & -2.209786 \\ \mathrm{H} & 0.668173 & -2.616445 & -0.757440 \\ \mathrm{C} & 0.542553 & -3.702003 & -0.793735 \\ \mathrm{H} & 0.852669 & -2.269491 & -1.775553 \\ \mathrm{H} & 1.838478 & -2.256084 & 0.148642 \\ \mathrm{C} & 1.644165 & -2.608775 & 1.162932 \\ \mathrm{H} & 2.750703 & -2.743398 & -0.208644 \\ \mathrm{H} & 2.835444 & -0.240124 & -0.887838 \\ \mathrm{C} & 2.395207 & -0.558048 & -1.835276 \\ \mathrm{H} & 3.840717 & -0.671692 & -0.831653 \\ \mathrm{H} & 2.924565 & 1.283162 & -0.846753 \\ \mathrm{C} & & & \end{array}$




$\begin{array}{lrrr}\mathrm{H} & 3.237413 & 1.615961 & 0.148901 \\ \mathrm{C} & 1.661555 & 2.022752 & -1.280121 \\ \mathrm{H} & 1.902012 & 3.072847 & -1.476751 \\ \mathrm{H} & 1.280492 & 1.594340 & -2.209746 \\ \mathrm{C} & -0.667905 & 2.616312 & -0.757932 \\ \mathrm{H} & -0.852142 & 2.269256 & -1.776059 \\ \mathrm{H} & -0.542313 & 3.701871 & -0.794282 \\ \mathrm{C} & -1.838425 & 2.255942 & 0.147870 \\ \mathrm{H} & -1.644378 & 2.608617 & 1.162217 \\ \mathrm{H} & -2.750568 & 2.743232 & -0.209651 \\ \mathrm{Cl} & -0.001113 & 0.000445 & 2.760083 \\ \mathrm{H} & 3.722860 & 1.581897 & -1.530556 \\ \mathrm{Zn} & 0.000233 & 0.000045 & 0.433551 \\ \mathrm{H} & 2.445650 & -0.549274 & 1.092800 \\ \mathrm{H} & -0.877791 & -2.424058 & 0.561646 \\ \mathrm{H} & -2.445324 & 0.549002 & 1.091994 \\ \mathrm{H} & 0.877765 & 2.424230 & 0.561517 \\ ----------------------------------------------------\end{array}$

$\mathrm{E}(\mathrm{RTPSSh})=-2854.4176099$ Hartree

Zero-point correction $=0.360704$

Thermal correction to Energy $=0.378279$

Thermal correction to Enthalpy $=0.379223$

Thermal correction to Gibbs Free Energy $=0.316001$

Sum of electronic and zero-point Energies $=-2854.056906$

Sum of electronic and thermal Energies $=-2854.039331$

Sum of electronic and thermal Enthalpies $=-2854.038387$

Sum of electronic and thermal Free Energies = -2854.101609

Table S25. Cartesian coordinates $(\AA)$ of $[\mathrm{Zn}(\text { cyclam }) \mathrm{Cl}]^{+}($trans-III $)$optimized at the rTPSSh/Def2-TZVPP level (scrf=pcm).

40

$\begin{array}{lrrr}\text { ZncyclamCl } & & & \\ \mathrm{H} & 1.172638 & 3.642440 & -0.687344 \\ \mathrm{C} & -0.800325 & 2.772233 & -0.567963 \\ \mathrm{H} & -0.931485 & 2.698209 & -1.649753 \\ \mathrm{H} & 0.832892 & 2.835641 & 0.851648 \\ \mathrm{C} & 0.686907 & 2.775855 & -0.229052 \\ \mathrm{H} & -3.470192 & 2.126751 & -0.373378 \\ \mathrm{H} & -1.594696 & 1.777858 & 1.031483 \\ \mathrm{~N} & -1.460368 & 1.592445 & 0.038997 \\ \mathrm{C} & -2.784642 & 1.292579 & -0.556558 \\ \mathrm{H} & -2.642333 & 1.216813 & -1.636981 \\ \mathrm{H} & 1.249751 & 1.485516 & -1.704840 \\ \mathrm{~N} & 1.284177 & 1.505053 & -0.687218 \\ \mathrm{H} & 2.699179 & 1.280862 & 0.825075 \\ \mathrm{C} & 2.689894 & 1.300194 & -0.266692 \\ \mathrm{H} & -4.451737 & -0.002344 & -0.318156 \\ \mathrm{C} & -3.399598 & -0.001678 & -0.022719 \\ \mathrm{H} & -3.393576 & -0.002201 & 1.072502 \\ \mathrm{Zn} & -0.021343 & 0.000015 & 0.108497 \\ \mathrm{H} & 3.192006 & 0.001666 & -1.919925 \\ \mathrm{C} & 3.271759 & 0.001387 & -0.826654 \\ \mathrm{H} & 4.341433 & 0.001936 & -0.603270 \\ \mathrm{C} & -2.782597 & -1.294524 & -0.557657 \\ \mathrm{H} & -2.639260 & -1.217285 & -1.637841 \\ \mathrm{~N} & -1.458572 & -1.593563 & 0.038994 \\ \mathrm{H} & 2.701333 & -1.279902 & 0.824388 \\ \mathrm{C} & 2.691428 & -1.298412 & -0.267390 \\ \mathrm{H} & -1.593562 & -1.778511 & 1.031475 \\ \mathrm{~N} & 1.285712 & -1.504261 & -0.687220 \\ & & & \end{array}$




\begin{tabular}{|c|c|c|c|}
\hline $\mathrm{H}$ & 1.250847 & -1.485231 & -1.704841 \\
\hline $\mathrm{H}$ & 3.308752 & -2.141201 & -0.597515 \\
\hline $\mathrm{C}$ & -0.797662 & -2.773338 & -0.566972 \\
\hline $\mathrm{H}$ & -0.929189 & -2.700428 & -1.648785 \\
\hline $\mathrm{H}$ & 0.835839 & -2.834391 & 0.852423 \\
\hline $\mathrm{C}$ & 0.689635 & -2.775366 & -0.228288 \\
\hline $\mathrm{H}$ & -1.262577 & -3.706000 & -0.236899 \\
\hline $\mathrm{H}$ & -1.266098 & 3.704855 & -0.238952 \\
\hline $\mathrm{H}$ & -3.467348 & -2.129724 & -0.376169 \\
\hline $\mathrm{H}$ & 1.176101 & -3.641754 & -0.686174 \\
\hline $\mathrm{H}$ & 3.306599 & 2.143810 & -0.595865 \\
\hline $\mathrm{Cl}$ & 0.462914 & 0.000640 & 2.381622 \\
\hline \multicolumn{4}{|c|}{$\mathrm{E}(\mathrm{RTPSSh})=-2854.417915$ Hartree } \\
\hline \\
\hline \multicolumn{4}{|c|}{ Thermal correction to Energy $=0$} \\
\hline \multicolumn{4}{|c|}{ Thermal correction to Enthalpy $=0.378756$} \\
\hline \multicolumn{4}{|c|}{ Thermal correction to Gibbs Free Energy $=0.315444$} \\
\hline \multicolumn{4}{|c|}{ Sum of electronic and zero-point Energies $=-2854.057798$} \\
\hline \multicolumn{4}{|c|}{ Sum of electronic and thermal Energies $=-2854.040103$} \\
\hline \multicolumn{4}{|c|}{ Sum of electronic and ther } \\
\hline Sum of & electronic and therma. & Free Energi & $e s=-2854.102471$ \\
\hline
\end{tabular}

Table S26. Cartesian coordinates $(\AA)$ of $[\mathrm{Zn}(\mathbf{c y c l a m}) \mathrm{Cl}]^{+}($cis-V) optimized at the rTPSSh/Def2-TZVPP level (scrf=pcm).

40

\section{ZncyclamCl}

$\begin{array}{lrrr}\text { H } & 4.121960 & 1.140748 & -0.936935 \\ \mathrm{H} & 3.938159 & -1.079390 & -0.128826 \\ \mathrm{H} & 3.314830 & 0.005416 & 1.113637 \\ \mathrm{C} & 3.101600 & 0.751903 & -0.890517 \\ \mathrm{H} & 2.463739 & 2.793941 & -1.159714 \\ \mathrm{C} & 3.117576 & -0.396674 & 0.118106 \\ \mathrm{H} & 2.272176 & 2.203908 & 0.493976 \\ \mathrm{H} & 2.878791 & 0.384215 & -1.897509 \\ \mathrm{C} & 2.179235 & 1.924419 & -0.557697 \\ \mathrm{~N} & 1.845105 & -1.155899 & 0.214766 \\ \mathrm{H} & 1.855557 & -1.648064 & 1.105091 \\ \mathrm{H} & 2.334040 & -2.987323 & -0.769846 \\ \mathrm{H} & 1.810486 & -1.667802 & -1.817083 \\ \mathrm{~N} & 0.757428 & 1.587835 & -0.811862 \\ \mathrm{H} & 0.000250 & 3.148239 & 0.354612 \\ \mathrm{H} & 0.008260 & 3.507840 & -1.376984 \\ \mathrm{H} & 0.690853 & 1.288883 & -1.783496 \\ \mathrm{C} & 1.632444 & -2.152267 & -0.855181 \\ \mathrm{C} & -0.175601 & 2.724467 & -0.636416 \\ \mathrm{Zn} & -0.003366 & -0.000882 & 0.408356 \\ \mathrm{C} & 0.197996 & -2.662962 & -0.790420 \\ \mathrm{H} & 0.009464 & -3.140215 & 0.173701 \\ \mathrm{C} & -1.608736 & 2.216978 & -0.749526 \\ \mathrm{H} & 0.022853 & -3.404508 & -1.574791 \\ \mathrm{H} & -0.652190 & -1.164522 & -1.867046 \\ \mathrm{H} & -2.313229 & 3.045192 & -0.630528 \\ \mathrm{H} & -1.770237 & 1.784365 & -1.738808 \\ \mathrm{~N} & -0.732405 & -1.517403 & -0.915022 \\ \mathrm{~N} & -1.838410 & 1.162478 & 0.261937 \\ \mathrm{C} & -2.157605 & -1.868157 & -0.699401 \\ \mathrm{H} & -2.839771 & -0.251569 & -1.956537 \\ \mathrm{H} & -2.263676 & -2.206396 & 0.333752 \\ & & & \end{array}$




$\begin{array}{lrrr}\mathrm{C} & -3.108564 & 0.409119 & 0.098325 \\ \mathrm{H} & -2.433879 & -2.702182 & -1.353081 \\ \mathrm{H} & -3.321809 & -0.050547 & 1.065130 \\ \mathrm{C} & -3.075910 & -0.677950 & -0.976088 \\ \mathrm{H} & -3.924822 & 1.105898 & -0.121720 \\ \mathrm{H} & -4.095257 & -1.063215 & -1.059659 \\ \mathrm{H} & -1.871415 & 1.607114 & 1.176293 \\ \mathrm{Cl} & -0.057106 & -0.163287 & 2.734697 \\ -\end{array}$

$\mathrm{E}(\mathrm{RTPSSh})=-2854.4148082$ Hartree

Zero-point correction $=0.361098$

Thermal correction to Energy $=0.378465$

Thermal correction to Enthalpy $=0.379409$

Thermal correction to Gibbs Free Energy $=0.317311$

Sum of electronic and zero-point Energies $=-2854.053710$

Sum of electronic and thermal Energies $=-2854.036343$

Sum of electronic and thermal Enthalpies $=-2854.035399$

Sum of electronic and thermal Free Energies = -2854.097498

Table S27. Cartesian coordinates $(\AA)$ of $[\mathrm{Zn}(\mathbf{T M C}) \mathrm{Cl}]^{+}$(trans-I) optimized at the rTPSSh/Def2-TZVPP level (scrf=pcm).

52

\section{$\mathrm{ZnTMCCl}$}

N
N
N
N
C
H
H
C
H
H
C
H
H
C
H
H
C
H
H
C
H
H
C
H
C
H
H
C
H
H
C
H
H
Cl
H
Zn

$$
\begin{array}{r}
-2.025295 \\
-0.728806 \\
2.025295 \\
0.728807 \\
-2.708746 \\
-2.105050 \\
-3.671117 \\
-2.940836 \\
-3.597984 \\
-3.495876 \\
-1.693153 \\
-1.991035 \\
-1.147011 \\
0.473072 \\
0.265131 \\
0.673362 \\
1.678309 \\
1.477861 \\
2.531674 \\
2.708803 \\
2.105166 \\
3.671196 \\
2.940847 \\
3.495864 \\
1.693145 \\
1.991013 \\
1.147002 \\
-0.473060 \\
-0.673345 \\
-0.265111 \\
-1.678307 \\
-1.477869 \\
-2.531670 \\
-0.000038 \\
3.598002 \\
0.000006
\end{array}
$$

0.964682

$-1.915216$

$-0.964674$

1. 915211

0.528901

0.850622

1.053544

$-0.974151$

$-1.126042$

$-1.355561$

$-1.805380$

$-2.820366$

$-1.369313$

$-2.638337$

$-3.712110$

$-2.288598$

$-2.404249$

$-2.756089$

$-2.977485$

$-0.528902$

$-0.850669$

$-1.053513$

0.974155

1. 355592

1. 805349

2.820327

1. 369245

2. 638357

2.288656

3. 712130

2. 404255

2. 756092

2. 977493

0.000010

1. 126053

$-0.000007$
0.046817

$-0.444364$

0.046828

$-0.444365$

$-1.200652$

$-2.050271$

$-1.266694$

$-1.309248$

$-2.169812$

$-0.450555$

$-1.578364$

$-1.870437$

$-2.417185$

$-0.939768$

$-1.002235$

$-1.952112$

$-0.049403$

0.963356

$-0.431771$

$-1.200614$

$-2.050257$

$-1.266584$

$-1.309228$

$-0.450532$

$-1.578368$

$-1.870479$

$-2.417171$

$-0.939760$

$-1.952118$

$-1.002189$

$-0.049411$

0.963351

$-0.431784$

2.638534

$-2.169786$

0.337258 


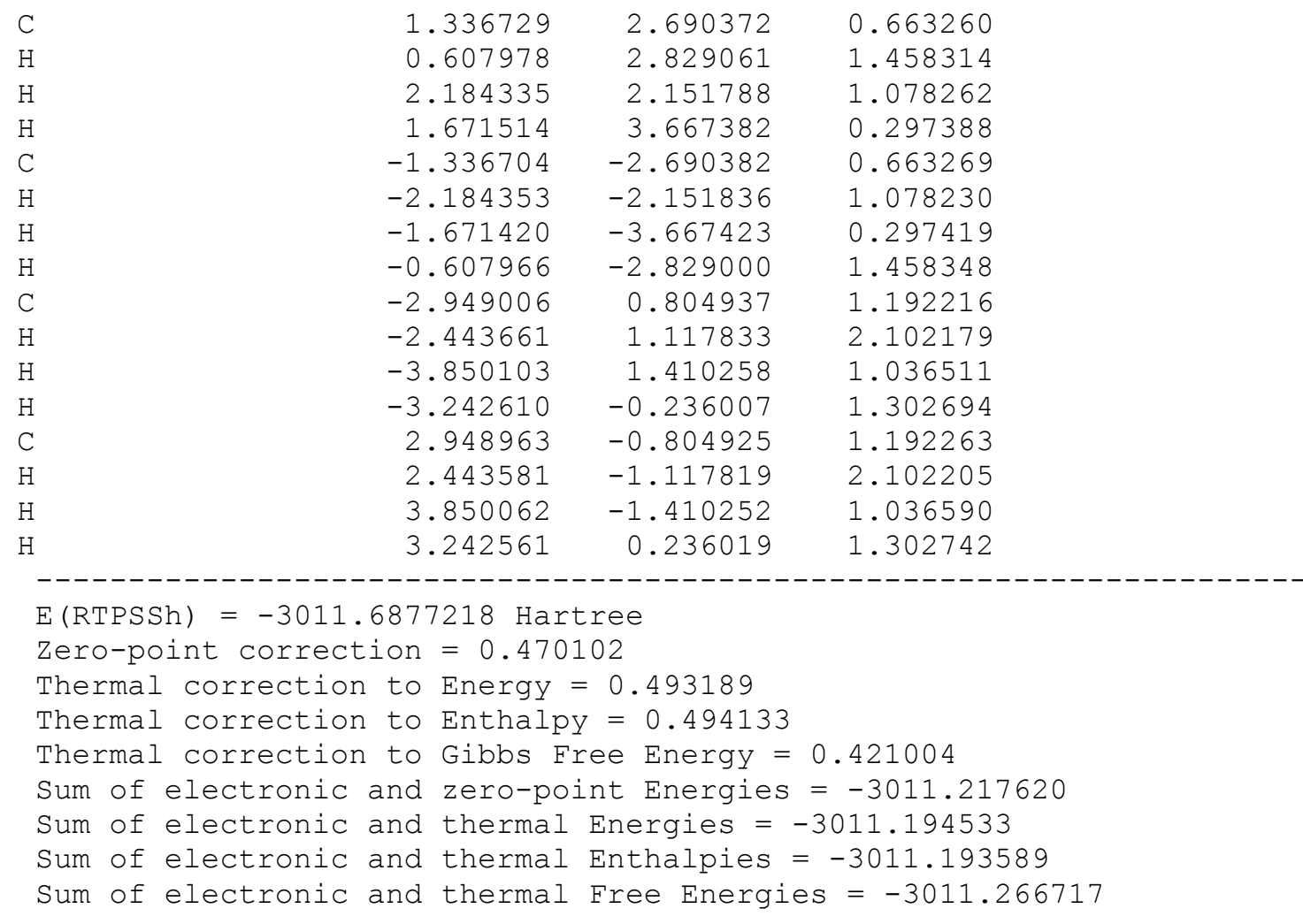

Table S28. Cartesian coordinates $(\AA)$ of $[\mathrm{Zn}(\mathbf{T M C}) \mathrm{Cl}]^{+}$(trans-III) optimized the rTPSSh/Def2-TZVPP level (scrf=pcm).

\begin{tabular}{|c|c|c|c|}
\hline \multicolumn{4}{|c|}{52} \\
\hline \multicolumn{4}{|l|}{$\mathrm{Z}_{1}$} \\
\hline $\mathrm{H}$ & -1.220412 & -3.672260 & -0.121106 \\
\hline C & 0.720875 & -2.806702 & -0.491516 \\
\hline $\mathrm{H}$ & 0.666786 & -2.858218 & -1.577948 \\
\hline $\mathrm{H}$ & -0.617368 & -2.648001 & 1.195777 \\
\hline$C$ & -0.677067 & -2.748435 & 0.110310 \\
\hline $\mathrm{H}$ & 3.202641 & -2.090388 & -1.286056 \\
\hline$N$ & 1.511378 & -1.600160 & -0.115638 \\
\hline$C$ & 2.483410 & -1.266544 & -1.196083 \\
\hline $\mathrm{H}$ & 1.922158 & -1.216362 & -2.131192 \\
\hline$N$ & -1.410537 & -1.567348 & -0.405425 \\
\hline $\mathrm{H}$ & -2.356918 & -1.340957 & 1.435121 \\
\hline$C$ & -2.653537 & -1.342676 & 0.385970 \\
\hline $\mathrm{H}$ & 4.032538 & 0.051702 & -1.759672 \\
\hline$C$ & 3.248651 & 0.040614 & -0.997473 \\
\hline $\mathrm{H}$ & 3.776717 & 0.046889 & -0.042014 \\
\hline $\mathrm{Zr}$ & 0.021395 & -0.000164 & 0.146071 \\
\hline $\mathrm{H}$ & -3.754059 & -0.045306 & -0.968622 \\
\hline$C$ & -3.397942 & -0.043638 & 0.063650 \\
\hline $\mathrm{H}$ & -4.305719 & -0.059235 & 0.673751 \\
\hline$C$ & 2.449035 & 1.327289 & -1.194332 \\
\hline $\mathrm{H}$ & 1.889961 & 1.263668 & -2.129962 \\
\hline$N$ & 1.466790 & 1.632819 & -0.114495 \\
\hline $\mathrm{H}$ & -2.388581 & 1.271010 & 1.442727 \\
\hline & -2.690561 & 1.273658 & 0.395117 \\
\hline & -1.459457 & 1.537901 & -0.401652 \\
\hline & -3.396120 & 2.101297 & 0.244496 \\
\hline & 0.646223 & 2.820319 & -0.487384 \\
\hline & 0.585979 & 2.869389 & -1.573637 \\
\hline
\end{tabular}




\begin{tabular}{|c|c|c|c|}
\hline $\mathrm{H}$ & -0.680476 & 2.621501 & 1.205000 \\
\hline $\mathrm{C}$ & -0.747657 & 2.729267 & 0.120633 \\
\hline $\mathrm{H}$ & 1.138500 & 3.737293 & -0.150306 \\
\hline $\mathrm{H}$ & 1.232561 & -3.711946 & -0.151698 \\
\hline $\mathrm{H}$ & 3.145813 & 2.170394 & -1.281621 \\
\hline $\mathrm{H}$ & -1.310169 & 3.644160 & -0.100534 \\
\hline $\mathrm{H}$ & -3.334369 & -2.188620 & 0.224237 \\
\hline $\mathrm{Cl}$ & -0.239539 & -0.007637 & 2.468572 \\
\hline $\mathrm{C}$ & 2.175590 & 1.944278 & 1.148578 \\
\hline $\mathrm{H}$ & 1.452078 & 2.249740 & 1.900230 \\
\hline $\mathrm{H}$ & 2.696182 & 1.067903 & 1.523895 \\
\hline $\mathrm{H}$ & 2.898231 & 2.750553 & 0.979483 \\
\hline $\mathrm{C}$ & -1.742949 & -1.785270 & -1.830860 \\
\hline $\mathrm{H}$ & -2.261885 & -2.742847 & -1.954842 \\
\hline $\mathrm{H}$ & -2.390962 & -0.993524 & -2.190376 \\
\hline $\mathrm{H}$ & -0.843491 & -1.784291 & -2.441440 \\
\hline C & -1.810111 & 1.765722 & -1.821217 \\
\hline $\mathrm{H}$ & -2.338005 & 2.720291 & -1.929896 \\
\hline $\mathrm{H}$ & -0.918299 & 1.777681 & -2.442965 \\
\hline $\mathrm{H}$ & -2.457516 & 0.973215 & -2.180586 \\
\hline C & 2.228561 & -1.897201 & 1.145872 \\
\hline $\mathrm{H}$ & 1.512221 & -2.214971 & 1.899398 \\
\hline $\mathrm{H}$ & 2.965558 & -2.690350 & 0.976280 \\
\hline $\mathrm{H}$ & 2.734390 & -1.011245 & 1.519118 \\
\hline \\
\hline \multicolumn{4}{|c|}{ Zero-point correction $=0.470615$} \\
\hline \multicolumn{4}{|c|}{ Thermal correction to Energy $=0.493344$} \\
\hline \multicolumn{4}{|c|}{ Thermal correction to Enthalpy $=0.494288$} \\
\hline \multicolumn{4}{|c|}{ Thermal correction to Gibbs Free Energy $=0.422655$} \\
\hline \multicolumn{4}{|c|}{ Sum of electronic and zero-point Energies $=-3011.212960$} \\
\hline \multicolumn{4}{|c|}{ Sum of electronic and thermal Energies $=-3011.190231$} \\
\hline \multicolumn{4}{|c|}{ Sum of electronic and thermal Enthalpies $=-3011.189287$} \\
\hline Sum of electronic a & and therma. & Free Energi & $e s=-3011.260920$ \\
\hline
\end{tabular}

Table S29. Cartesian coordinates $(\AA)$ of $[\mathrm{Zn}(\mathbf{T M C}) \mathrm{Cl}]^{+}($cis-V) optimized at the rTPSSh/Def2-TZVPP level (scrf=pcm).

52

\section{$\mathrm{ZnTMCCl}$}

$\mathrm{H}$
$\mathrm{H}$
$\mathrm{H}$
$\mathrm{C}$
$\mathrm{H}$
$\mathrm{C}$
$\mathrm{H}$
$\mathrm{H}$
$\mathrm{C}$
$\mathrm{N}$
$\mathrm{H}$
$\mathrm{H}$
$\mathrm{N}$
$\mathrm{H}$
$\mathrm{H}$
$\mathrm{C}$
$\mathrm{C}$
$\mathrm{Zn}$
$\mathrm{C}$
$\mathrm{H}$

$$
\begin{array}{r}
3.556212 \\
4.079607 \\
3.066935 \\
2.744536 \\
1.499816 \\
3.063772 \\
1.290365 \\
2.825777 \\
1.439359 \\
2.146891 \\
3.146629 \\
2.210109 \\
0.240588 \\
-0.929244 \\
-1.146729 \\
2.212704 \\
-1.016749 \\
0.000203 \\
1.016317 \\
0.929098
\end{array}
$$$$
2.398411
$$$$
0.212115
$$$$
1.031393
$$$$
1.672598
$$$$
3.400002
$$$$
0.581543
$$$$
2.672817
$$$$
1.299605
$$$$
2.446762
$$$$
-0.593255
$$$$
-2.079617
$$$$
-0.913469
$$$$
1.701804
$$$$
2.941501
$$$$
\text { 3. } 241829
$$$$
-1.506923
$$$$
\text { 2. } 455845
$$$$
0.000087
$$$$
-2.456878
$$$$
-2.941726
$$

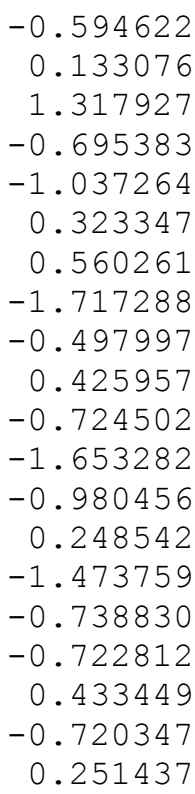




\begin{tabular}{|c|c|c|c|}
\hline $\mathrm{C}$ & -2.213117 & 1.505884 & -0.740050 \\
\hline $\mathrm{H}$ & 1.146043 & -3.243463 & -1.470711 \\
\hline $\mathrm{H}$ & -3.147089 & 2.078520 & -0.726123 \\
\hline $\mathrm{H}$ & -2.210704 & 0.911420 & -1.653840 \\
\hline $\mathrm{N}$ & -0.241010 & -1.702946 & -0.978288 \\
\hline N & -2.146892 & 0.593484 & 0.425709 \\
\hline C & -1.439765 & -2.447706 & -0.495228 \\
\hline $\mathrm{H}$ & -2.826664 & -1.301889 & -1.715238 \\
\hline $\mathrm{H}$ & -1.290646 & -2.673078 & 0.563164 \\
\hline C & -3.063694 & -0.581516 & 0.324722 \\
\hline $\mathrm{H}$ & -1.500150 & -3.401267 & -1.033916 \\
\hline $\mathrm{H}$ & -3.066381 & -1.030292 & 1.319793 \\
\hline C & -2.744943 & -1.673704 & -0.692945 \\
\hline $\mathrm{H}$ & -4.079645 & -0.212351 & 0.134544 \\
\hline $\mathrm{H}$ & -3.556593 & -2.399387 & -0.590974 \\
\hline $\mathrm{Cl}$ & 0.000723 & 0.002601 & 2.818199 \\
\hline C & 2.600266 & -1.345508 & 1.623549 \\
\hline $\mathrm{H}$ & 3.635058 & -1.674911 & 1.472486 \\
\hline $\mathrm{H}$ & 2.536091 & -0.703031 & 2.495388 \\
\hline $\mathrm{H}$ & 1.968634 & -2.212905 & 1.795245 \\
\hline $\mathrm{C}$ & -2.600000 & 1.346922 & 1.622672 \\
\hline $\mathrm{H}$ & -3.634941 & 1.675875 & 1.471630 \\
\hline $\mathrm{H}$ & -2.535320 & 0.705390 & 2.495170 \\
\hline $\mathrm{H}$ & -1.968550 & 2.214677 & 1.793197 \\
\hline C & -0.357748 & -1.557426 & -2.449730 \\
\hline $\mathrm{H}$ & -0.564511 & -2.538653 & -2.891416 \\
\hline $\mathrm{H}$ & -1.159145 & -0.878158 & -2.719608 \\
\hline $\mathrm{H}$ & 0.572027 & -1.182516 & -2.865430 \\
\hline C & 0.357177 & 1.555240 & -2.451753 \\
\hline $\mathrm{H}$ & 1.158597 & 0.875834 & -2.721231 \\
\hline $\mathrm{H}$ & -0.572622 & 1.179945 & -2.867087 \\
\hline $\mathrm{H}$ & 0.563831 & 2.536170 & -2.894169 \\
\hline \\
\hline \multicolumn{4}{|c|}{ Zero-point correction $=0.471279$} \\
\hline \multicolumn{4}{|c|}{ Thermal correction to Energy $=0.493776$} \\
\hline \multicolumn{4}{|c|}{ Thermal correction to Enthalpy $=0.494720$} \\
\hline \multicolumn{4}{|c|}{ Thermal correction to Gibbs Free Energy $=0.422900$} \\
\hline \multicolumn{4}{|c|}{ Sum of electronic and zero-point Energies $=-3011.194810$} \\
\hline \multicolumn{4}{|c|}{ Sum of electronic and thermal Energies $=-3011.172312$} \\
\hline \multicolumn{4}{|c|}{ Sum of electronic and $t$} \\
\hline Sum of electronic & and thermal & Free Energi & $e s=-3011.243188$ \\
\hline
\end{tabular}

Table S30. Cartesian coordinates $(\AA)$ of $\left[\mathrm{Zn}(\mathbf{C B} \text {-cyclam-E)Cl }]^{+}(\right.$anti) optimized at the rTPSSh/Def2-TZVPP level ( $\mathrm{scrf}=\mathrm{pcm})$.

51

\section{ZnCBcyclamECl}

$\mathrm{C}$
$\mathrm{H}$
$\mathrm{H}$
$\mathrm{C}$
$\mathrm{H}$
$\mathrm{C}$
$\mathrm{H}$
$\mathrm{H}$
$\mathrm{C}$
$\mathrm{H}$
$\mathrm{H}$
$\mathrm{C}$

$$
\begin{array}{r}
-2.154809 \\
-2.976387 \\
-2.117517 \\
-2.441801 \\
-2.354029 \\
-1.526621 \\
-1.943223 \\
-1.536430 \\
0.658667 \\
0.424577 \\
0.326751 \\
2.166894
\end{array}
$$$$
\begin{array}{r}
1.044080 \\
1.719563 \\
0.977047 \\
-0.352466 \\
-0.333504 \\
-1.453062 \\
-2.427865 \\
-1.397207 \\
-2.399284 \\
-2.171850 \\
-3.423859 \\
-2.296725
\end{array}
$$$$
\begin{array}{r}
-0.641852 \\
-0.384294 \\
-1.731550 \\
-0.070639 \\
1.020290 \\
-0.629014 \\
-0.346888 \\
-1.720282 \\
-1.043417 \\
-2.084595 \\
-0.840292 \\
-0.819840
\end{array}
$$ 


\begin{tabular}{|c|c|c|c|}
\hline $\mathrm{H}$ & 2.677517 & -2.870457 & -1.596279 \\
\hline $\mathrm{H}$ & 2.449138 & -2.744051 & 0.132150 \\
\hline $\mathrm{C}$ & 3.746103 & -0.616349 & 0.105171 \\
\hline $\mathrm{H}$ & 4.533964 & -1.362064 & -0.045001 \\
\hline $\mathrm{H}$ & 4.156682 & 0.356604 & -0.171603 \\
\hline $\mathrm{C}$ & 3.345148 & -0.599562 & 1.579592 \\
\hline $\mathrm{C}$ & 2.503664 & 0.592040 & 2.032420 \\
\hline $\mathrm{H}$ & 2.973814 & 1.513089 & 1.681350 \\
\hline $\mathrm{H}$ & 2.491167 & 0.625919 & 3.128776 \\
\hline $\mathrm{C}$ & 0.508190 & 1.950902 & 1.838196 \\
\hline $\mathrm{H}$ & 1.197653 & 2.694964 & 1.436508 \\
\hline $\mathrm{H}$ & 0.431194 & 2.112890 & 2.919596 \\
\hline $\mathrm{C}$ & -0.869184 & 2.120463 & 1.196782 \\
\hline $\mathrm{H}$ & -1.625635 & 1.565760 & 1.750070 \\
\hline $\mathrm{H}$ & -1.155907 & 3.173059 & 1.249328 \\
\hline $\mathrm{C}$ & 0.264449 & -0.469725 & 2.129397 \\
\hline $\mathrm{H}$ & -0.711463 & -0.051656 & 2.359759 \\
\hline $\mathrm{H}$ & 0.694880 & -0.791518 & 3.082548 \\
\hline $\mathrm{C}$ & 0.087315 & -1.710305 & 1.238354 \\
\hline $\mathrm{H}$ & 0.955944 & -2.354951 & 1.340288 \\
\hline $\mathrm{H}$ & -0.766642 & -2.282712 & 1.613071 \\
\hline $\mathrm{N}$ & -0.869411 & 1.638517 & -0.204017 \\
\hline $\mathrm{N}$ & 1.098496 & 0.617016 & 1.540983 \\
\hline $\mathrm{N}$ & -0.097571 & -1.421405 & -0.212199 \\
\hline $\mathrm{N}$ & 2.615766 & -0.884806 & -0.820347 \\
\hline $\mathrm{H}$ & -0.666786 & 2.420977 & -0.820306 \\
\hline $\mathrm{H}$ & 2.917085 & -0.635000 & -1.758063 \\
\hline $\mathrm{Cl}$ & 1.520557 & 1.777605 & -2.403633 \\
\hline $\mathrm{Zn}$ & 0.905445 & 0.440481 & -0.599278 \\
\hline $\mathrm{H}$ & 4.272820 & -0.553901 & 2.156406 \\
\hline C & -3.897487 & -0.741365 & -0.405655 \\
\hline $\mathrm{H}$ & -4.096826 & -0.547769 & -1.464721 \\
\hline $\mathrm{H}$ & -4.026898 & -1.815586 & -0.249094 \\
\hline C & -4.939098 & -0.022498 & 0.437499 \\
\hline $\mathrm{H}$ & -4.883987 & 1.062770 & 0.303296 \\
\hline $\mathrm{H}$ & -4.776396 & -0.242340 & 1.499374 \\
\hline 0 & -6.224504 & -0.500366 & 0.021340 \\
\hline $\mathrm{H}$ & -6.896212 & -0.027147 & 0.525888 \\
\hline $\mathrm{H}$ & 2.874832 & -1.542076 & 1.870750 \\
\hline
\end{tabular}

$\mathrm{E}(\mathrm{RTPSSh})=-3085.7402299$ Hartree

Zero-point correction $=0.456752$

Thermal correction to Energy $=0.478991$

Thermal correction to Enthalpy $=0.479935$

Thermal correction to Gibbs Free Energy $=0.407032$

Sum of electronic and zero-point Energies $=-3085.283478$

Sum of electronic and thermal Energies $=-3085.261239$

Sum of electronic and thermal Enthalpies $=-3085.260295$

Sum of electronic and thermal Free Energies = -3085.333198

Table S31. Cartesian coordinates $(\AA)$ of $[\mathrm{Zn}(\mathbf{C B}-\text { cyclam-E }) \mathrm{Cl}]^{+}($syn $)$optimized at the rTPSSh/Def2-TZVPP level ( $\mathrm{scrf}=\mathrm{pcm})$.

51

\section{ZnCBcyclamE}

$\mathrm{C}$

$\mathrm{H}$

$\mathrm{H}$

C

$\mathrm{H}$

$\begin{array}{ll}3.089719 & 1.658077 \\ 3.509013 & 2.664699 \\ 3.518139 & 1.045932 \\ 3.501608 & 1.082445 \\ 3.077788 & 1.667939\end{array}$

0.700080

0.796008

1.495963

$-0.658754$

$-1.477482$ 


\begin{tabular}{|c|c|c|c|}
\hline $\mathrm{C}$ & 3.277639 & -0.416643 & -0.888913 \\
\hline $\mathrm{H}$ & 3.833186 & -0.722651 & -1.783578 \\
\hline $\mathrm{H}$ & 3.682647 & -0.974736 & -0.042067 \\
\hline $\mathrm{C}$ & 1.726915 & -2.316380 & -0.977848 \\
\hline $\mathrm{H}$ & 2.299464 & -2.652753 & -0.111879 \\
\hline $\mathrm{H}$ & 2.157434 & -2.786175 & -1.869105 \\
\hline $\mathrm{C}$ & 0.263803 & -2.746237 & -0.835533 \\
\hline $\mathrm{H}$ & 0.231890 & -3.823836 & -0.657205 \\
\hline $\mathrm{H}$ & -0.283732 & -2.564890 & -1.759482 \\
\hline $\mathrm{C}$ & -1.897853 & -1.849038 & 0.093359 \\
\hline $\mathrm{H}$ & -2.213558 & -2.385970 & -0.805250 \\
\hline $\mathrm{H}$ & -2.384220 & -2.331340 & 0.942143 \\
\hline $\mathrm{C}$ & -2.393605 & -0.394833 & 0.005195 \\
\hline $\mathrm{H}$ & -2.163320 & 0.113054 & 0.949872 \\
\hline $\mathrm{C}$ & -1.748419 & 0.381277 & -1.154796 \\
\hline $\mathrm{H}$ & -2.406022 & 1.190812 & -1.488732 \\
\hline $\mathrm{H}$ & -1.631500 & -0.293519 & -2.005522 \\
\hline $\mathrm{C}$ & -0.493525 & 2.295606 & -0.226017 \\
\hline $\mathrm{H}$ & -1.171624 & 2.214219 & 0.625214 \\
\hline $\mathrm{H}$ & -0.915919 & 3.028271 & -0.923431 \\
\hline $\mathrm{C}$ & 0.884386 & 2.778351 & 0.249844 \\
\hline $\mathrm{H}$ & 1.488459 & 3.116986 & -0.591016 \\
\hline $\mathrm{H}$ & 0.749000 & 3.641933 & 0.903335 \\
\hline $\mathrm{C}$ & 0.442624 & 0.991468 & -2.069476 \\
\hline $\mathrm{H}$ & 1.127548 & 1.827959 & -1.981218 \\
\hline $\mathrm{H}$ & -0.170480 & 1.184239 & -2.955247 \\
\hline $\mathrm{C}$ & 1.226995 & -0.305103 & -2.279428 \\
\hline $\mathrm{H}$ & 0.559939 & -1.070380 & -2.670632 \\
\hline $\mathrm{H}$ & 1.993503 & -0.140015 & -3.042481 \\
\hline $\mathrm{N}$ & 1.622609 & 1.699760 & 0.953040 \\
\hline $\mathrm{N}$ & -0.405792 & 0.952808 & -0.850994 \\
\hline $\mathrm{N}$ & 1.855648 & -0.834555 & -1.035946 \\
\hline $\mathrm{N}$ & -0.420490 & -1.999740 & 0.248480 \\
\hline $\mathrm{H}$ & 4.581884 & 1.236145 & -0.733400 \\
\hline $\mathrm{C}$ & -3.924782 & -0.424756 & -0.157785 \\
\hline $\mathrm{H}$ & -4.339303 & -1.221705 & 0.466289 \\
\hline $\mathrm{H}$ & 1.491926 & 1.809836 & 1.954388 \\
\hline $\mathrm{H}$ & -0.241698 & -2.469670 & 1.130818 \\
\hline $\mathrm{Cl}$ & 0.908670 & -0.783362 & 2.905101 \\
\hline $\mathrm{H}$ & -4.180015 & -0.668577 & -1.194456 \\
\hline C & -4.600868 & 0.879838 & 0.229888 \\
\hline $\mathrm{H}$ & -4.209705 & 1.718093 & -0.357552 \\
\hline $\mathrm{H}$ & -4.417352 & 1.094603 & 1.289214 \\
\hline O & -6.004890 & 0.726165 & -0.011250 \\
\hline $\mathrm{H}$ & -6.441145 & 1.551828 & 0.228906 \\
\hline $\mathrm{Zn}$ & 0.692262 & -0.196828 & 0.632997 \\
\hline \multicolumn{4}{|c|}{$\mathrm{E}(\mathrm{RTPSSh})=-3085.736785$ Hartree } \\
\hline \multicolumn{4}{|c|}{ Zero-point correction $=0.457139$} \\
\hline \multicolumn{4}{|c|}{ Thermal correction to Energy $=0.479339$} \\
\hline \multicolumn{4}{|c|}{ Thermal correction to Enthalpy $=0.480283$} \\
\hline \multicolumn{4}{|c|}{ Thermal correction to Gibbs Free Energy $=0.406731$} \\
\hline \multicolumn{4}{|c|}{ Sum of electronic and zero-point Energies $=-3085.279646$} \\
\hline Sum of electronic a & and thermal & Energies $=$ & -3085.257446 \\
\hline Sum of electronic a & and thermal & Enthalpies & $=-3085.256502$ \\
\hline Sum of electronic a & and thermal & Free Energi & $e s=-3085.330054$ \\
\hline
\end{tabular}

Table S32. Cartesian coordinates $(\AA)$ of $[\mathrm{Zn}(\mathbf{C B}-\mathrm{DMC}-\mathbf{E}) \mathrm{Cl}]^{+}$(anti) optimized the rTPSSh/Def2-TZVPP level ( $\mathrm{scrf}=\mathrm{pcm})$. 


\section{ZnCBDMCECl}

\begin{tabular}{|c|c|c|c|}
\hline $\mathrm{C}$ & -2.226794 & 0.228795 & 0.996042 \\
\hline $\mathrm{H}$ & -3.144606 & -0.160566 & 1.453503 \\
\hline $\mathrm{H}$ & -2.108834 & 1.263505 & 1.324431 \\
\hline $\mathrm{C}$ & -2.383467 & 0.217336 & -0.535376 \\
\hline $\mathrm{H}$ & -2.323243 & -0.806254 & -0.917821 \\
\hline C & -1.357906 & 1.082118 & -1.284932 \\
\hline $\mathrm{H}$ & -1.704764 & 1.226550 & -2.315110 \\
\hline $\mathrm{H}$ & -1.300629 & 2.063599 & -0.809118 \\
\hline C & 0.945077 & 1.601892 & -1.856536 \\
\hline $\mathrm{H}$ & 0.759148 & 2.498168 & -1.263046 \\
\hline $\mathrm{H}$ & 0.713975 & 1.828226 & -2.903678 \\
\hline C & 2.410162 & 1.194766 & -1.741930 \\
\hline $\mathrm{H}$ & 3.031236 & 2.037827 & -2.058213 \\
\hline $\mathrm{H}$ & 2.633320 & 0.378547 & -2.426177 \\
\hline $\mathrm{C}$ & 3.778980 & -0.333355 & -0.399545 \\
\hline $\mathrm{H}$ & 4.639369 & -0.007554 & -0.998635 \\
\hline $\mathrm{H}$ & 4.133005 & -0.468623 & 0.624854 \\
\hline $\mathrm{C}$ & 3.279492 & -1.670840 & -0.933356 \\
\hline $\mathrm{H}$ & 4.163765 & -2.305806 & -1.035683 \\
\hline $\mathrm{C}$ & 2.311331 & -2.421314 & -0.029969 \\
\hline $\mathrm{H}$ & 2.693284 & -2.414611 & 0.993063 \\
\hline $\mathrm{H}$ & 2.252996 & -3.468258 & -0.352813 \\
\hline $\mathrm{C}$ & 0.184730 & -2.595854 & 1.079863 \\
\hline $\mathrm{H}$ & 0.788097 & -2.542144 & 1.987511 \\
\hline $\mathrm{H}$ & 0.071098 & -3.653819 & 0.816345 \\
\hline $\mathrm{C}$ & -1.183339 & -1.979440 & 1.324537 \\
\hline $\mathrm{H}$ & -1.842681 & -2.152714 & 0.476791 \\
\hline $\mathrm{H}$ & -1.650314 & -2.471713 & 2.182424 \\
\hline $\mathrm{C}$ & 0.229188 & -1.998181 & -1.289167 \\
\hline $\mathrm{H}$ & -0.775251 & -2.362901 & -1.093777 \\
\hline $\mathrm{H}$ & 0.720392 & -2.766147 & -1.893052 \\
\hline $\mathrm{C}$ & 0.144238 & -0.705591 & -2.124711 \\
\hline $\mathrm{H}$ & 1.025943 & -0.623902 & -2.754248 \\
\hline $\mathrm{H}$ & -0.709390 & -0.790502 & -2.802535 \\
\hline $\mathrm{N}$ & -1.072390 & -0.519688 & 1.564060 \\
\hline $\mathrm{N}$ & 0.931836 & -1.867539 & 0.020020 \\
\hline $\mathrm{N}$ & 0.030686 & 0.548834 & -1.331184 \\
\hline $\mathrm{N}$ & 2.785432 & 0.776051 & -0.360670 \\
\hline $\mathrm{Cl}$ & 0.540674 & 2.326777 & 1.668693 \\
\hline Zn & 0.792852 & 0.254962 & 0.611720 \\
\hline $\mathrm{H}$ & 2.877117 & -1.579642 & -1.945168 \\
\hline $\mathrm{C}$ & -3.795527 & 0.742597 & -0.877956 \\
\hline $\mathrm{H}$ & -4.014865 & 1.633844 & -0.281373 \\
\hline $\mathrm{H}$ & -3.823027 & 1.047233 & -1.927295 \\
\hline $\mathrm{C}$ & -4.894666 & -0.286278 & -0.663181 \\
\hline $\mathrm{H}$ & -4.920260 & -0.632327 & 0.375670 \\
\hline $\mathrm{H}$ & -4.720126 & -1.157129 & -1.306263 \\
\hline 0 & -6.138247 & 0.340143 & -1.000713 \\
\hline $\mathrm{H}$ & -6.843772 & -0.301090 & -0.856578 \\
\hline C & 3.438025 & 1.915306 & 0.326091 \\
\hline $\mathrm{H}$ & 3.626010 & 1.652758 & 1.365883 \\
\hline $\mathrm{H}$ & 4.388072 & 2.151760 & -0.168027 \\
\hline $\mathrm{H}$ & 2.783512 & 2.781559 & 0.300402 \\
\hline $\mathrm{C}$ & -1.037326 & -0.278866 & 3.025175 \\
\hline $\mathrm{H}$ & -0.874972 & 0.780128 & 3.208752 \\
\hline $\mathrm{H}$ & -1.979844 & -0.599973 & 3.483812 \\
\hline $\mathrm{H}$ & -0.216948 & -0.845388 & 3.465617 \\
\hline
\end{tabular}

$E($ RTPSSh $)=-3164.3738789$ Hartree 
Zero-point correction $=0.511834$

Thermal correction to Energy $=0.536674$

Thermal correction to Enthalpy $=0.537618$

Thermal correction to Gibbs Free Energy $=0.460272$

Sum of electronic and zero-point Energies $=-3163.862044$

Sum of electronic and thermal Energies $=-3163.837205$

Sum of electronic and thermal Enthalpies $=-3163.836261$

Sum of electronic and thermal Free Energies $=-3163.913607$

Table S33. Cartesian coordinates $(\AA)$ of $[\mathrm{Zn}(\mathbf{C B}-\mathrm{DMC}-\mathrm{E}) \mathrm{Cl}]^{+}($syn $)$optimized at the rTPSSh/Def2-TZVPP level ( $\mathrm{scrf}=\mathrm{pcm})$.

57

\section{ZnCBDMCECl}

\begin{tabular}{|c|c|c|c|}
\hline C & -3.196195 & -1.485705 & 0.306417 \\
\hline $\mathrm{H}$ & -3.757552 & -2.426271 & 0.376196 \\
\hline $\mathrm{H}$ & -3.614509 & -0.793419 & 1.039185 \\
\hline $\mathrm{C}$ & -3.382696 & -0.905390 & -1.097806 \\
\hline $\mathrm{H}$ & -2.928824 & -1.539357 & -1.861369 \\
\hline $\mathrm{C}$ & -3.012330 & 0.565215 & -1.299351 \\
\hline $\mathrm{H}$ & -3.405265 & 0.901896 & -2.266096 \\
\hline $\mathrm{H}$ & -3.502842 & 1.151491 & -0.520346 \\
\hline $\mathrm{C}$ & -1.445609 & 2.388006 & -1.037558 \\
\hline $\mathrm{H}$ & -2.070940 & 2.632613 & -0.179349 \\
\hline $\mathrm{H}$ & -1.831783 & 2.927235 & -1.910510 \\
\hline $\mathrm{C}$ & -0.007614 & 2.841321 & -0.776265 \\
\hline $\mathrm{H}$ & -0.047209 & 3.852409 & -0.365170 \\
\hline $\mathrm{H}$ & 0.543929 & 2.914237 & -1.712629 \\
\hline $\mathrm{C}$ & 2.074832 & 1.576103 & -0.419187 \\
\hline $\mathrm{H}$ & 2.010874 & 1.634324 & -1.506663 \\
\hline $\mathrm{H}$ & 2.803064 & 2.332840 & -0.107340 \\
\hline $\mathrm{C}$ & 2.575142 & 0.177091 & -0.040535 \\
\hline $\mathrm{H}$ & 2.290422 & -0.049717 & 0.993183 \\
\hline $\mathrm{C}$ & 1.991045 & -0.871932 & -0.993288 \\
\hline $\mathrm{H}$ & 2.501382 & -1.827052 & -0.857670 \\
\hline $\mathrm{H}$ & 2.202858 & -0.548281 & -2.015075 \\
\hline C & 0.314200 & -2.498434 & -0.339070 \\
\hline $\mathrm{H}$ & 0.882689 & -2.558509 & 0.590582 \\
\hline $\mathrm{H}$ & 0.722460 & -3.245284 & -1.029644 \\
\hline $\mathrm{C}$ & -1.151585 & -2.805514 & -0.076179 \\
\hline $\mathrm{H}$ & -1.697681 & -2.920034 & -1.010106 \\
\hline $\mathrm{H}$ & -1.223826 & -3.768128 & 0.437978 \\
\hline $\mathrm{C}$ & -0.182718 & -0.960033 & -2.185132 \\
\hline $\mathrm{H}$ & -0.975374 & -1.697609 & -2.244939 \\
\hline $\mathrm{H}$ & 0.500974 & -1.175038 & -3.010607 \\
\hline $\mathrm{C}$ & -0.766853 & 0.439556 & -2.384478 \\
\hline $\mathrm{H}$ & 0.046251 & 1.145661 & -2.540898 \\
\hline $\mathrm{H}$ & -1.372637 & 0.447724 & -3.296265 \\
\hline $\mathrm{N}$ & -1.791663 & -1.740564 & 0.735164 \\
\hline $\mathrm{N}$ & 0.516343 & -1.125136 & -0.877767 \\
\hline $\mathrm{N}$ & -1.566257 & 0.917087 & -1.226396 \\
\hline $\mathrm{N}$ & 0.743777 & 1.941040 & 0.154598 \\
\hline $\mathrm{H}$ & -4.457243 & -0.959357 & -1.295972 \\
\hline $\mathrm{C}$ & 4.113691 & 0.148755 & -0.120793 \\
\hline $\mathrm{H}$ & 4.506095 & 1.027716 & 0.397911 \\
\hline $\mathrm{Cl}$ & -1.883399 & 1.178890 & 2.223409 \\
\hline $\mathrm{H}$ & 4.434887 & 0.226499 & -1.165017 \\
\hline $\mathrm{C}$ & 4.748988 & -1.081910 & 0.507085 \\
\hline $\mathrm{H}$ & 4.451457 & -1.998136 & -0.013438 \\
\hline $\mathrm{H}$ & 4.437762 & -1.169752 & 1.554970 \\
\hline
\end{tabular}




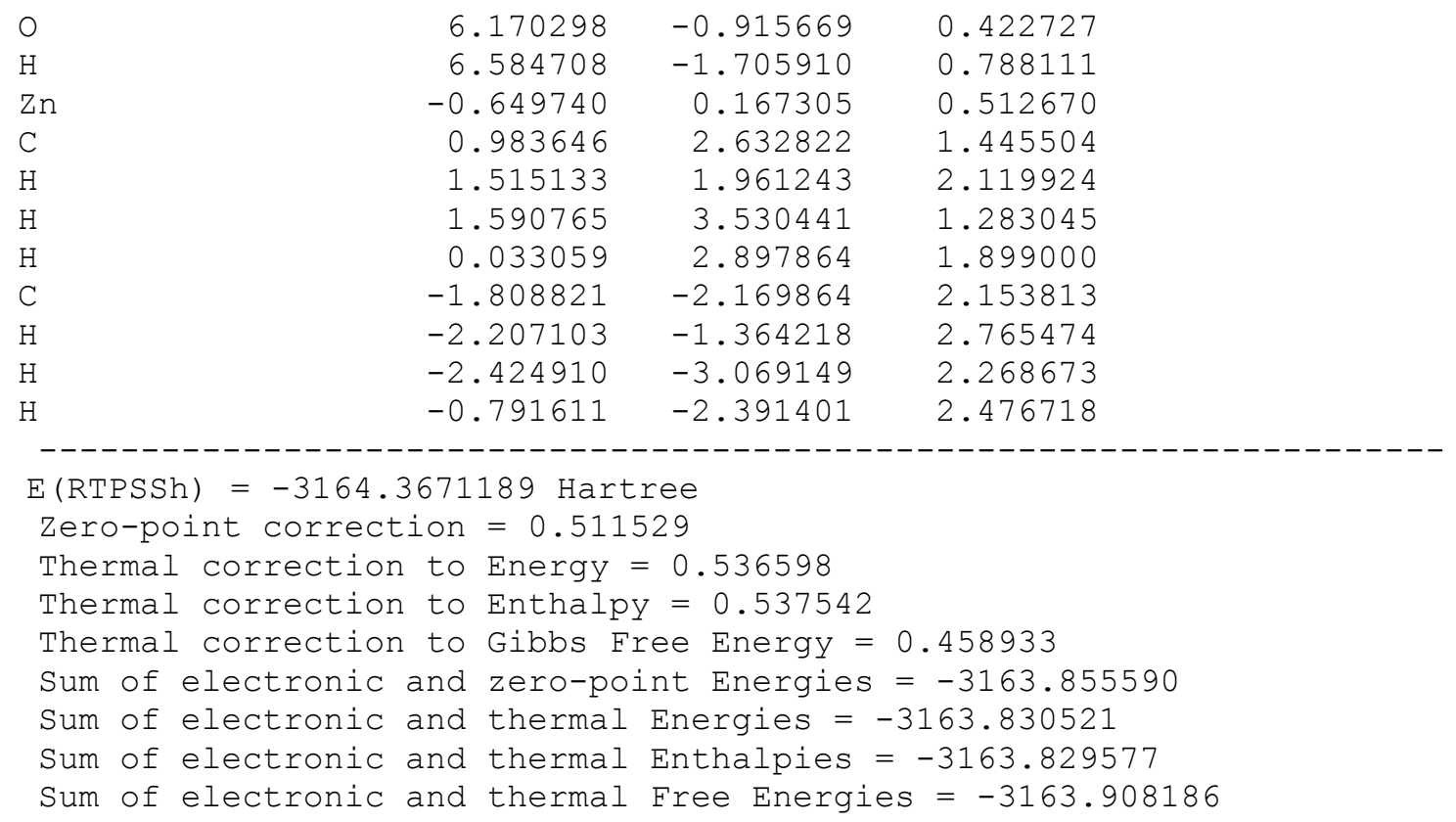

\section{References}

(1) Camus, N.; Halime, Z.; Le Bris, N.; Bernard, H.; Platas-Iglesias, C.; Tripier, R. Full Control of the Regiospecific $N$-Functionalization of $C$-Functionalized Cyclam Bisaminal Derivatives and Application to the Synthesis of Their TETA, TE2A, and CB-TE2A Analogues. J. Org. Chem. 2014, 79, 1885-1899.

(2) Tasker, P. A.; Sklar, L. Crystal and Molecular Structure of Di(Perchlorato) $(1,4,8,11-$ Tetraazacyclo-Tetradecane)Copper(II). Cu(Cyclam) $\left(\mathrm{ClO}_{4}\right)_{2}$. J. Cryst. Mol. Struct. 1975, 5, 329-344.

(3) Kim, G.; Lelong, E.; Kang, J.; Suh, J.-M.; Le Bris, N.; Bernard, H.; Kim, D.; Tripier, R.; Lim, M. H. Reactivities of Cyclam Derivatives with Metal-Amyloid- $\beta$. Inorg. Chem. Front. 2020, 7, 4222-4238.

(4) Lu, T. H.; Shui, W. Z.; Tung, S. F.; Chi, T. Y.; Liao, F. L.; Chung, C. S. (IsothiocyanatoKN)(1,4,8,11-Tetramethyl-1,4,8,11-Tetraazacyclotetradecane-K4N)Copper(II)

Perchlorate. Acta Cryst. 1998, C54, 1071-1072.

(5) Alcock, N. W.; Herron, N.; Moore, P. Comparison of the Structure and Dynamic Properties of Anion (1,4,8,11-Tetramethyl-1,4,8,11-Tetra-Azacyclotetradecane) Zinc(II) Perchlorate Complexes in Nitromethane Solutions, and the Crystal and Molecular Structure of the Chloro-Complex. J.C.S Dalton 1978, 1282-1288.

(6) Niu, W.; Wong, E. H.; R. Weisman, G.; Lam, K.-C.; Rheingold, A. L. Two Novel Zinc(II) Complexes of the 1,8-Cross-Bridged Cyclam Ligand and Their Structures. Inorg. Chem. Comm. 1999, 2, 358-360.

(7) Liang, X.; Weishäupl, M.; Parkinson, J. A.; Parsons, S.; McGregor, P. A.; Sadler, P. J. Selective Recognition of Configurational Substates of Zinc Cyclam by Carboxylates: 
Implications for the Design and Mechanism of Action of Anti-HIV Agents. Chem. Eur. J. 2003, 9, 4709-4717. 